\title{
Development of a method for calculation of cardiac output using Doppler ultrasound
}

\author{
Amit Diggikar \\ West Virginia University
}

Follow this and additional works at: https://researchrepository.wvu.edu/etd

\section{Recommended Citation}

Diggikar, Amit, "Development of a method for calculation of cardiac output using Doppler ultrasound" (1999). Graduate Theses, Dissertations, and Problem Reports. 986.

https://researchrepository.wvu.edu/etd/986

This Thesis is protected by copyright and/or related rights. It has been brought to you by the The Research Repository @ WVU with permission from the rights-holder(s). You are free to use this Thesis in any way that is permitted by the copyright and related rights legislation that applies to your use. For other uses you must obtain permission from the rights-holder(s) directly, unless additional rights are indicated by a Creative Commons license in the record and/ or on the work itself. This Thesis has been accepted for inclusion in WVU Graduate Theses, Dissertations, and Problem Reports collection by an authorized administrator of The Research Repository @ WVU. For more information, please contact researchrepository@mail.wvu.edu. 


\section{DEVELOPMENT OF A METHOD FOR CALCULATION OF CARDIAC OUTPUT USING DOPPLER ULTRASOUND}

By

Amit Diggikar, B.E.

THESIS

Submitted to

The College of Engineering and Mineral Resources at WEST VIRGINIA UNIVERSITY

in partial fulfillment of the requirements for the degree of Master of Science in Electrical Engineering

Committee:

Stephanie Schuckers, Ph.D., Chair

Wils L. Cooley, Ph.D.

Biswajit A. Das, Ph.D.

Department of Computer Science and Electrical Engineering

Morgantown, West Virginia,

1999. 


\section{ABSTRACT \\ DEVELOPMENT OF A METHOD FOR CALCULATION OF CARDIAC OUTPUT USING DOPPLER ULTRASOUND}

\section{By Amit Diggikar}

Keywords: Doppler shift signal, stroke volume, cardiac output, signal processing

Cardiac output, the amount of blood pumped by the heart in one minute, is one of the key parameters in the evaluation of the functionality of the heart. The principle of the Doppler effect can be used to measure cardiac output by measuring the peak frequency associated with the Doppler shift signal when an ultrasound beam is incident on the aorta. The peak frequency is directly proportional to the velocity of blood flowing in the aorta. Stroke volume is the product of the cross sectional area of the aorta and the integration of peak velocity over time. Stroke volume times the heart rate is the cardiac output. This project attempts to develop an algorithm that would automatically calculates the peak frequency from the Doppler shift signal and derive the cardiac output on a beat-by-beat basis. The difference in the power levels in the FFT of the signal when there is blood flow and when there is no blood flow is the major discriminating factor used in the peak frequency detection. However, Doppler ultrasound provides a non-invasive means of calculating the cardiac output. Eventually, this algorithm will be used to analyze data for understanding the mechanism for a cardiovascular abnormality called the orthostatic intolerance, which is found in astronauts returning from space as well as people on earth. 


\section{DEDICATION}

I dedicate this thesis to my family, especially my parents, for their infinite love, help and support throughout my life and education. 


\section{ACKNOWLEDGEMENT}

I wish to thank my advisor, Dr. Stephanie Schuckers, for her endless efforts and invaluable advice. Whenever I had a problem she was always there to answer with a smile. I would like to thank my committee members, Dr. Wils L. Cooley and Dr. Biswajit A. Das, for their precious time and help. I would also like to thank my colleagues at the Biomedical Signal Analysis Laboratory for their encouragement and support.

Special thanks to all my friends and roommates. I would like to thank my fiancé Deepthi for her infinite patience and love. 


\section{TABLE OF CONTENTS}

ABSTRACT ............................................................................................................................................II

CHAPTER 1: INTRODUCTION .................................................................................... 1

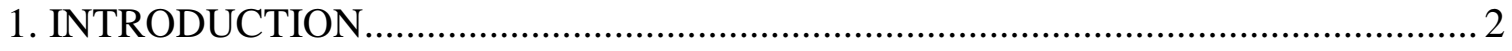

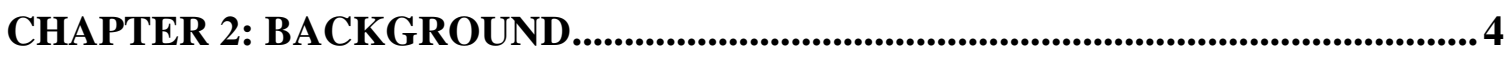

2. BACKGROUND

2.1 ULTRASOUND

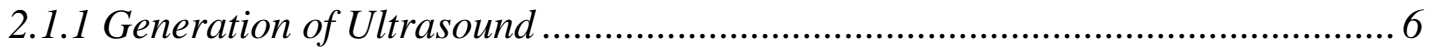

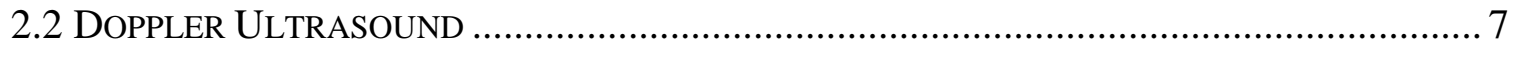

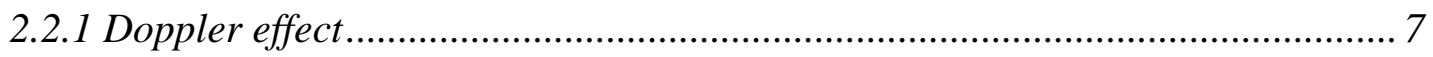

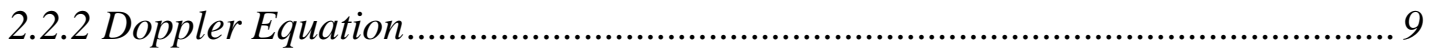

2.2.3 Doppler Instrumentation ........................................................................... 10

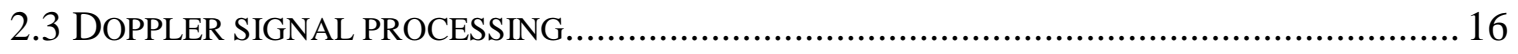

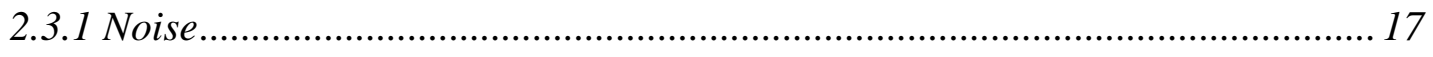

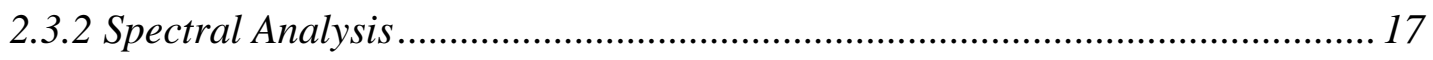

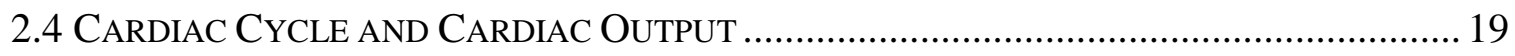

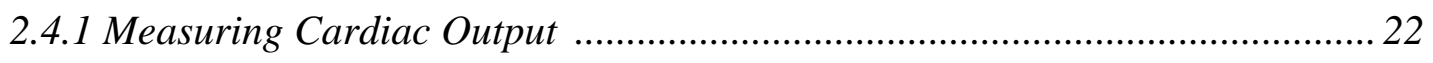

2.4.2 Cardiac output using ultrasound............................................................... 23 
CHAPTER 3: DIFFERENT APPROACHES ..................................................................25

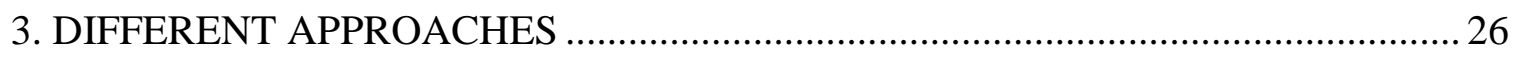

CHAPTER 4: PROCEDURE................................................................................................34

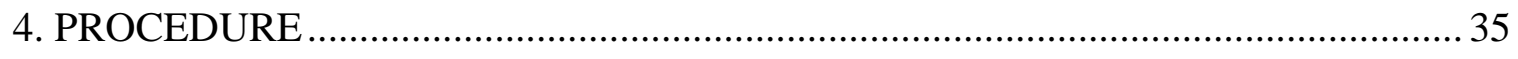

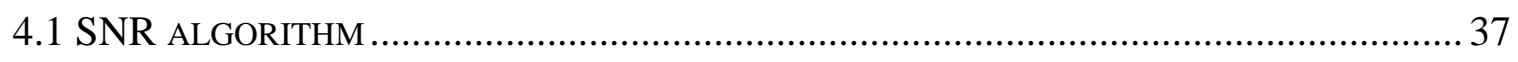

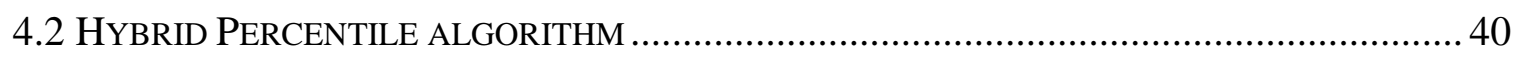

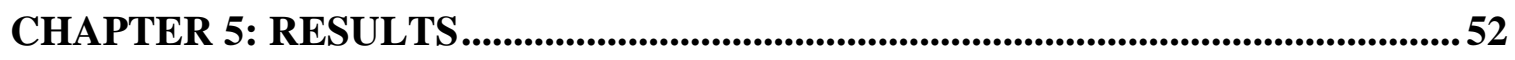

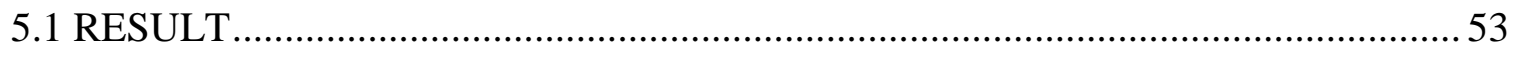

CHAPTER 6: CONCLUSION AND FUTURE WORK...............................................63

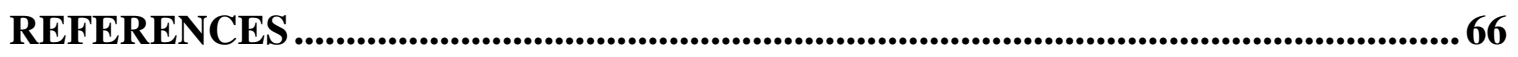

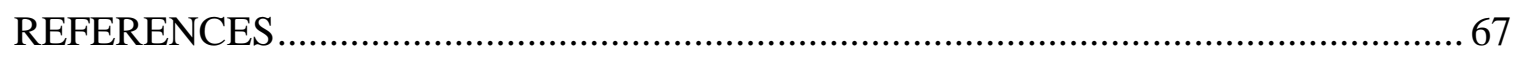

APPENDIX (1) : PROGRAMS .............................................................................................. 70

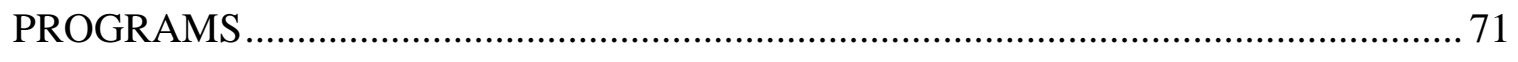

APPENDIX (2): FLOWCHARTS ..............................................................................................86

APPENDIX (3): DATA .........................................................................................................89 


\section{TABLE OF FIGURES}

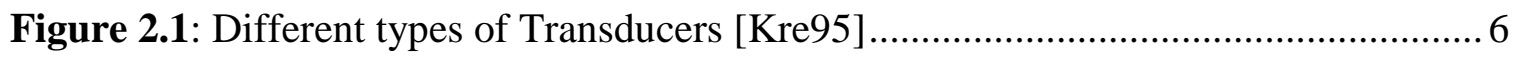

Figure 2.2: Demonstrating Doppler effect [Fei94] ...................................................... 8

Figure 2.3: Understanding Doppler equation ........................................................... 10

Figure 2.4: Basic Block diagram of Continuous-wave Doppler Instrument [Wey94]..... 11

Figure 2.5: Block Diagram of a plsed-wave Doppler instrument [Wey94] ..................... 12

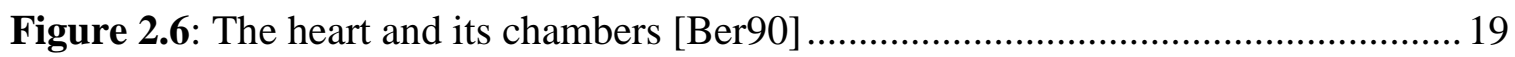

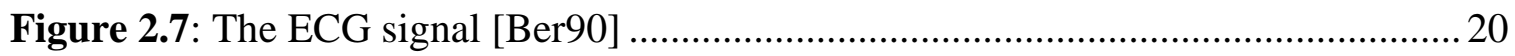

Figure 3.1: Fast Fourier transform of a noisy Doppler shift signal...............................28

Figure 3.2: Fast Fourier transform of a Doppler shift signal (when there is blood flow).29

Figure 3.3: Peak frequency location in the percentile method.......................................... 30

Figure 4.1: Typical data over a period of 5 seconds... ………………........................... 36

Figure 4.2: Basic concept of automated peak-frequency algorithm................................ 37

Figure 4.3: Typical ECG signal sampled as $12 \mathrm{kHz}$................................................. 41

Figure 4.4: Output of trigger program overlaid on the electrocardiogram...................... 42

Figure 4.5: Histogram used to determine noise vector.................................................. 43

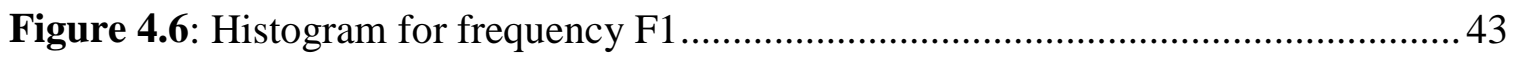

Figure 4.7: A typical calculated noise vector............................................................... 44

Figure 4.8: Cumulative sum of power (y-axis) for a typical block of signal when there is

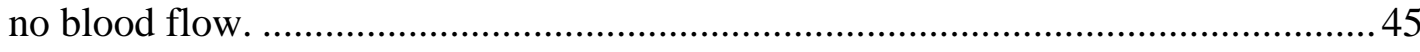

Figure 4.9: Cumulative sum of power (y-axis) for a typical block of signal when there is

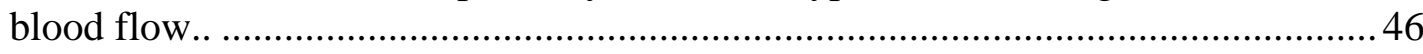

Figure 4.10: Peak frequency versus time (top) and smoothened velocity (bottom) where

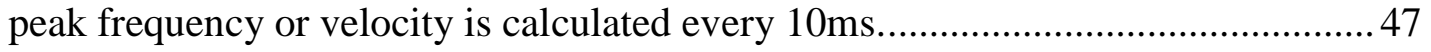


Figure 4.11: Beat to beat view of the output of the algorithm..

Figure 4.12: Cumulative sum of amplitudes curves............................................... 50

Figure 4.13: Cumulative sum of amplitudes over a complete cardiac cycle...................51

Figure 5.1: Lower cutoff = 50\% power @ 1000 Hz., Higher cutoff=80\% power @ 2000

Hz., Threshold $=95$ percentile.

Figure 5.2: Lower cutoff = 50\% power @ 1000 Hz., Higher cutoff =80\% power @ 2000

Hz., Threshold $=90$ percentile............................................................................ 55

Figure 5.3: Lower cutoff =50\% power @ 1000 Hz., Higher cutoff=80\% power @ 2000

Hz., Threshold = 93percentile ........................................................................ 56

Figure 5.4: Lower cutoff =60\% power @ 1000 Hz., Higher cutoff=90\% power @ 2000

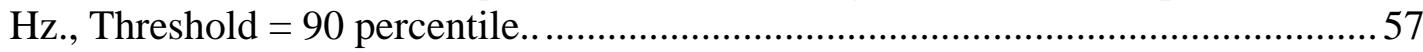

Figure 5.5: Lower cutoff=60\% power @ 1000 Hz., Higher cutoff=90\% power @ 2000

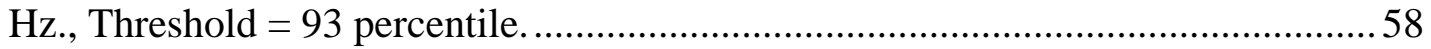

Figure 5.6: Lower cutoff =60\% power @ 1000 Hz., Higher cutoff=90\% power @ 2000

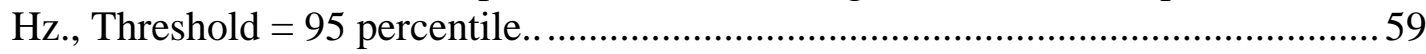

Figure 5.7: Heart rate comparison for referenced data versus calculated heart rate from

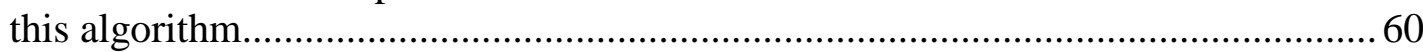

Figure 5.8: Comparison of stroke volume for the different parameter sets given in figures

5.1 through 5.6.. 61

Figure 5.9: Stroke volume comparison for best setting........................................... 62 


\section{CHAPTER 1: Introduction}




\section{INTRODUCTION}

Astronauts, returning from space, are often subject to a cardiovascular abnormality called orthostatic intolerance that may cause nausea, vomiting, lightheadedness or fainting. The exact cause of this abnormality is not established. The symptoms shown by the astronauts are similar to those in patients who have orthostatic intolerance due to autonomic dysfunction. Various studies are being undertaken on different data like electrocardiogram, manual blood pressure, beat-to-beat pressure, transcranial Doppler, plasma volume, norepinephrine, epinephrine, plasma renin activity, two-dimensional and M-mode echocardiography, and continuous-wave Doppler ultrasound for measuring aortic flow. [Fri96]

One of the variables of comparison in these studies for evaluating the capacity of the heart is the cardiac output, which is the volume of blood pumped by the heart in one minute. The ability of the heart to pump blood to meet the demands of body is of critical importance. Hence it is imperative to have a simple non-invasive technique to estimate the cardiac output. Doppler Ultrasound is one such technique that is reliable in estimating the cardiac output. This method is based on the measurement of the velocity of blood in the aorta. The main objective of this thesis is the analysis of the Doppler ultrasound measurement of aortic blood flow.

Doppler ultrasound can determine the presence or absence of flow, the direction, speed and the character of flow. The phenomenon whereby the frequency of the reflected sound is altered by movement of the reflected surface away from or toward the source is called the Doppler effect. This shift in frequency of the reflected ultrasound, when beamed on the aorta, contains information regarding the velocity of the moving blood cells. The amount by which the frequency is changed is called the Doppler shift frequency. 
This project involves application of signal processing techniques to develop an algorithm that minimizes noise and automates the estimation of beat-to-beat cardiac output. Cardiac output can be estimated from Doppler shift signal by determination of the beat-to-beat peak frequency, which is directly proportional to the velocity of blood in the aorta. The product of area under the velocity time curve and the cross sectional area of the aorta gives the stroke volume, i.e., the amount of blood flow per beat. Cardiac output is calculated by multiplying stroke volume by heart rate. 
CHAPTER 2: Background 


\section{BACKGROUND}

The field of ultrasound and its uses is a highly researched in engineering, medical and other scientific applications. Many studies have been undertaken to learn the natural phenomenon of Doppler effect in a better way so that they can be applied for better results. This chapter describes ultrasound in general and Doppler ultrasound in particular. Various books ([Wey94], [Nan93], [Sai93], [Fei95], and [Kre95]) have contributed to the information collected below.

Doppler ultrasound has been in use in diagnostic medicine for many years. Doppler ultrasound can determine the presence or absence of flow, the direction, speed and the character of flow. Long-standing applications include monitoring of the fetal heart rate during labor and delivery and evaluating blood flow in the carotid arteries. Doppler instruments provide audible and visual outputs of blood flow information.

\subsection{Ultrasound}

Sound at frequency greater than $20 \mathrm{kHz}$ is called ultrasound. Ultrasound is absorbed by biological media, reflected at tissue interfaces, and backscattered by blood cells. The degree of ultrasound absorption as it progresses through biological media depends on the properties of the medium and the frequency of ultrasound. Biological medium reduces ultrasound energy as it passes through the medium. The greater the water content of a medium, the lesser the ultrasound absorption.

When an ultrasonic beam intercepts tissue organ boundaries, the differences in acoustic characteristics of organs with respect to the media, results in the reflection of ultrasound back towards the source. These echoes can be measured yielding information about the location and other characteristics of tissue boundaries thus forming the basis of 
ultrasound scanning. An entirely different phenomenon occurs if ultrasound is incident on tissue inhomogeneities (e.g. red blood cells) whose size is comparable in wavelength to that of ultrasound beam. Although ultrasound is reflected back towards sources by blood cells, it is called backscatter and is different in behavior with respect to the echoes for macroscopic tissue interfaces at internal body organs.

\subsubsection{Generation of Ultrasound}

In ultrasonic instruments, rapidly vibrating surfaces like piezoelectric crystals are used to produce ultrasound. These crystals vibrate at a frequency in the range of 1 to 10 $\mathrm{MHz}$, upon applying voltage. These crystals also have the reverse property of generating an electric voltage when subjected to vibration. If ultrasound is incident upon a piezoelectric crystal, voltage fluctuations are produced corresponding to the incident waves' compression and rarefaction. These crystals can used to generate and detect ultrasound. Hence the same or a different crystal can be used as a transmitter or receiver in an ultrasound transducer.

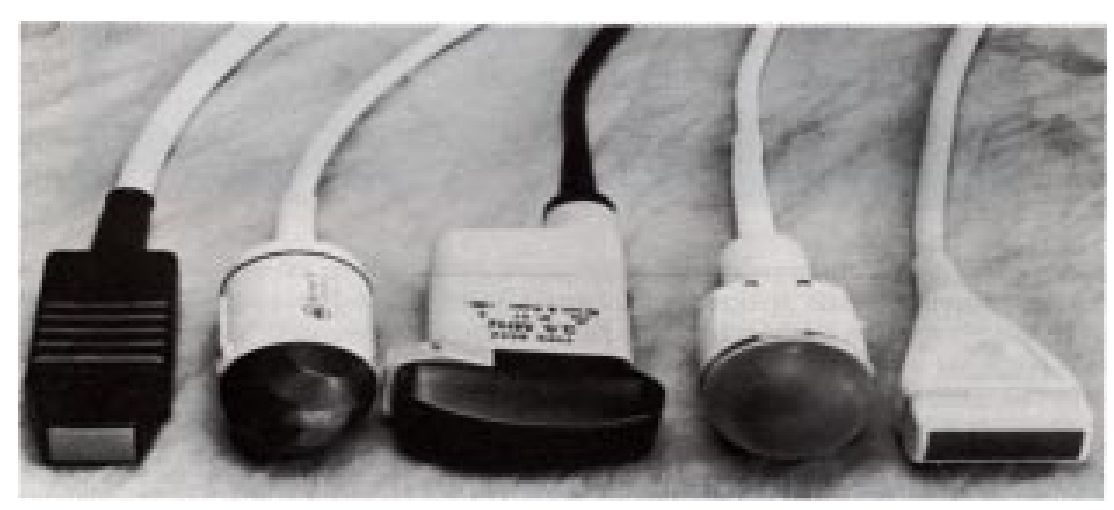

Figure 2.1: Different types of Transducers. [Kre95] 
The shape of the ultrasound beam is an important factor involved in the design of the transducer. A well-defined beam helps to focus on the organs or tissues of interest, thus minimizing the patients' total exposure to the ultrasound energy. Ultrasound beams may either be focused or unfocussed depending upon the area of body to be covered for scanning. The depth at which the beam is focused is also considered in the design of the transducer.

\subsection{Doppler Ultrasound}

The field of detection, quantisation, and medical evaluation of tissue motion and blood flow using ultrasound is known as Doppler ultrasound. Animals like bats and dolphins use the ultrasonic echo information to determine the motion of prey. Humans started investigating the nature of Doppler ultrasound during the middle of the nineteenth century. The pioneer in this study was Christian Johann Doppler. Doppler effect is used in many applications like measuring distance, velocity, weather forecasting, aviation safety, automatic door openers, and home burglar alarms.

\subsubsection{Doppler effect}

The phenomenon whereby the frequency of the reflected sound is altered by movement of the reflected surface away from or toward the source is called the Doppler effect. This shift in frequency of the reflected ultrasound contains information regarding the velocity of the moving medium. The amount by which the frequency is changed is called the Doppler shift frequency. The sign of this shift indicates the direction of movement of the reflecting surface. 


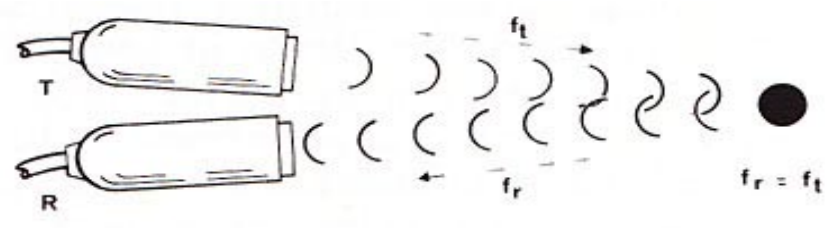

Target is stationary

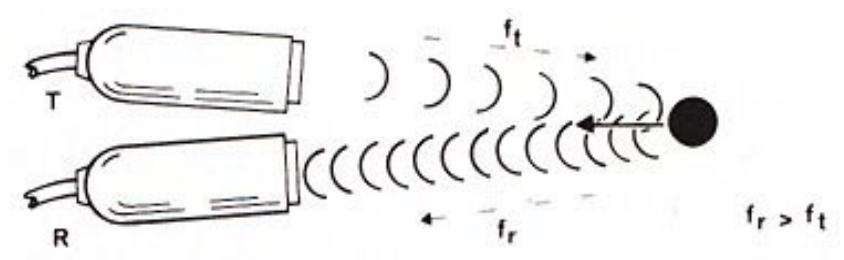

Target moving towards transducer

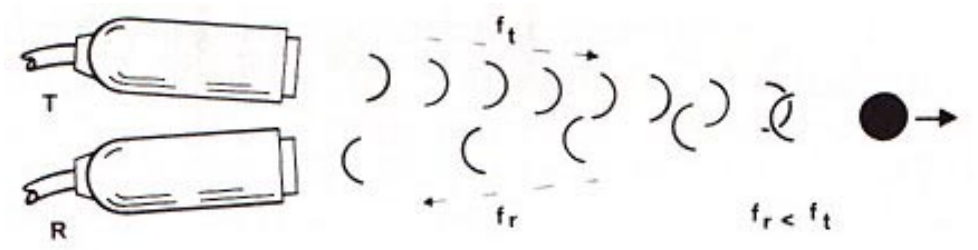

Target moving towards transducer

$\mathrm{f}_{\mathrm{r}}-$ received frequency

$\mathrm{f}_{\mathrm{t}}$ - transmitted frequency

$\mathrm{T}$ - Transmitter

$\mathrm{R}$ - Receiver

- Target

Figure 2.2: Demonstrating Doppler effect. [Fei94]

Doppler ultrasound is based on this phenomenon and is used in the body to determine presence and speed of blood flow. Reflections in Doppler ultrasound are due to moving blood cells. Since the speed of moving cells is much slower than that of the ultrasonic beam, the frequency shift is in the range of 0.5 to $10 \mathrm{kHz}$ for the ultrasonic 
frequency between 2 to $10 \mathrm{MHz}$. The ultrasonic beam at one frequency typically from 2 to $10 \mathrm{MHz}$ is reflected by a large number of blood cells that fall within the target volume insonated by the ultrasound beam. The reflected beam consists of a mixture of many frequencies of the range between 0.5 and $10 \mathrm{kHz}$, due to reflections from moving surfaces, each traveling with a different velocity. These are called the frequency components of the Doppler shift signals. Doppler instrumentation is capable of intercepting the backscattered ultrasound and computing the Doppler frequency shift signal by comparing the echo with the original transmitted frequency.

The phenomenon of constructive and destructive interference observed in sound is also seen in ultrasound. Thus, when ultrasound is reflected from many different blood cells and their net effect is measured at the transducer, the measured ultrasound intensity shows considerable variation. On an average over time, however, the Doppler frequency shift signal faithfully represents the velocity distribution of the blood cells intercepted by the beam.

\subsubsection{Doppler Equation}

If the orientation of the transducer with respect to blood vessels remains fixed, the amount of frequency shift produced is proportional to the velocity of the blood cells according to the Doppler equation. [Kre95]

$$
V=\frac{c \times \Delta f}{2 f_{t} \times \cos \phi}
$$

where

$\mathrm{V}=$ velocity of blood flow,

$\mathrm{f}=$ emitted frequency of ultrasound signal $\left(\mathrm{f}_{\mathrm{t}}\right)$ 
$\mathrm{c}=$ velocity of sound in tissue $(1540 \mathrm{~m} / \mathrm{s})$

$\Delta \mathrm{f}=$ Doppler frequency shift measured $\left(\mathrm{f}_{\mathrm{t}}-\mathrm{f}_{\mathrm{r}}\right)$

$\phi=$ angle of incidence between direction of blood flow and direction of emitted ultrasonic beam.

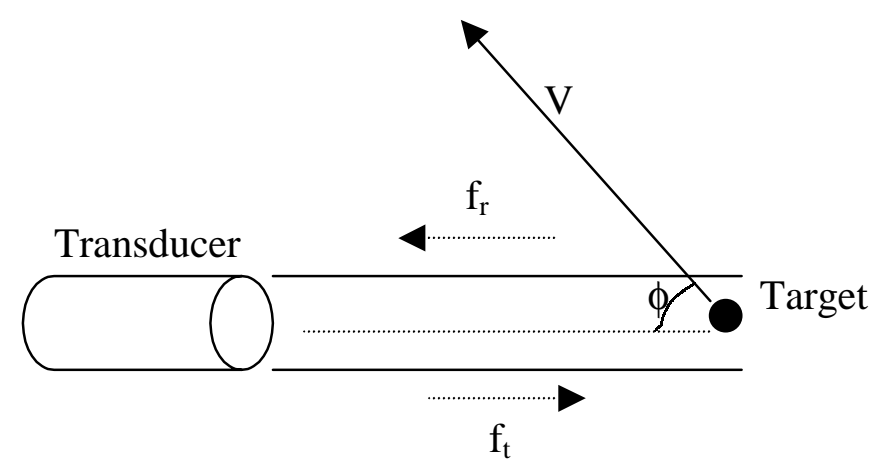

Figure 2.3: Understanding Doppler equation.

\subsubsection{Doppler Instrumentation}

Varieties of instruments have been developed to record the velocity of flow in the heart and great vessels using the Doppler principle. These include continuous-wave Doppler $(\mathrm{CW})$, pulsed-wave Doppler (PD), the high pulse repetition frequency Doppler (HiPRF), and the multigated Doppler.

\section{Continuous-Wave Doppler}

The basic components of a CW Doppler include the master oscillator, the transmitting amplifier, the transmitting and receiving transducers, the receiving amplifier, and the demodulator. The master oscillator produces a sinusoidal waveform, which is amplified and used to drive the transducer at its resonant frequency. Since the CW Doppler is continuously transmitting ultrasound, echoes will be constantly returning to 
the transducer. Due to the simultaneous movement of signal, the $\mathrm{CW}$ uses one transducer each for transmitting and receiving. The transmitting and receiving portion of the transducer are typically aligned such that their beam profiles overlap. The targets within these sample areas contribute to the Doppler output. The entire target within this beam reflects and backscatters echoes. These weak received echoes are initially pre-amplified and then transmitted to the demodulator, where the frequency of the received waveform is compared with a replica of the transmitted waveform derived directly from the oscillator (coherent demodulation) or from the transmitting transducer (non-coherent demodulation). The signal/noise ratio for the receiving amplifier is critical because the Doppler signal is 40 to $60 \mathrm{~dB}$ below the clutter (high intensity-low frequency noise). The output of the demodulator is the Doppler frequency shift. It is generally in the audible range, hence it can be output to an audio amplifier for auditory analysis. Various signal processing readouts can be added to convert the raw Doppler signal to an analog or digital signal for flow velocity analysis.

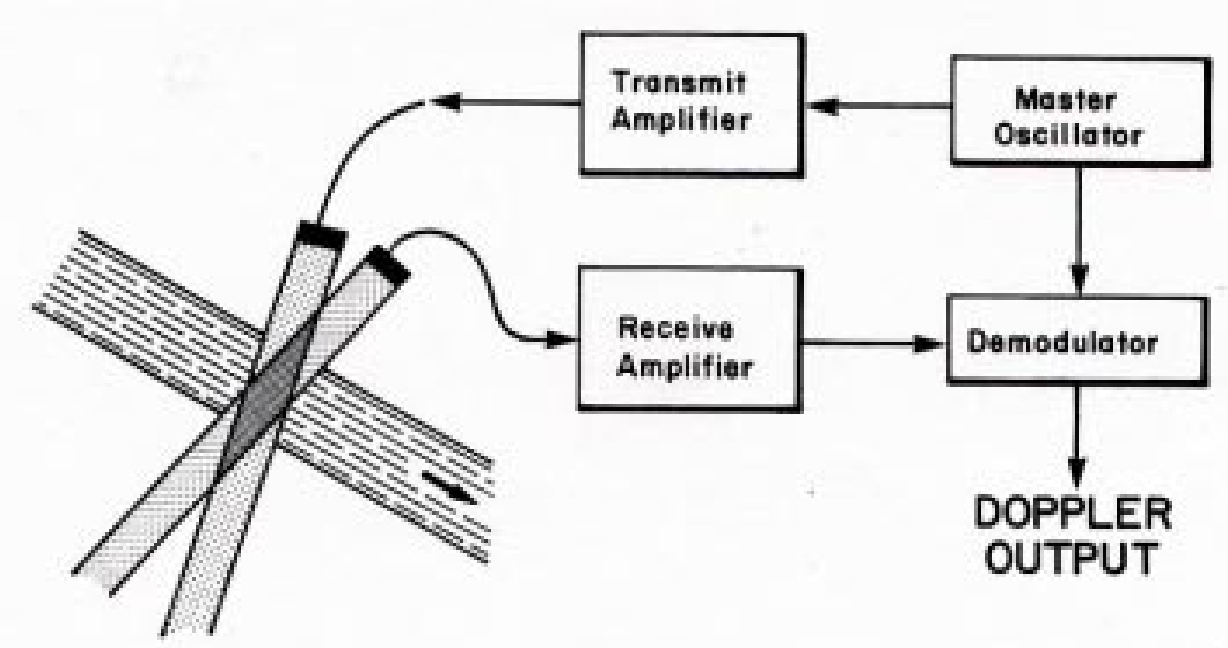

Figure 2.4: Basic Block diagram of Continuous-wave Doppler Instrument. [Wey94] 


\section{Pulsed Doppler}

The principle of operation of the pulsed Doppler is to transmit a short burst of sound energy towards the target and then record the returning echoes. Since the sound waves travel at a uniform speed through the soft tissue, the time delay between the transmission of the pulse and detection of an echo depends upon the range of the target. Because of this constant relationship between the time and distance, these two parameters become interchangeable. If the instrument only analyzes and outputs signals received during a specific time window following pulse transmission, the resulting Doppler shifts can originate only from targets moving at a range from the transducer that corresponds to the selected time delay.

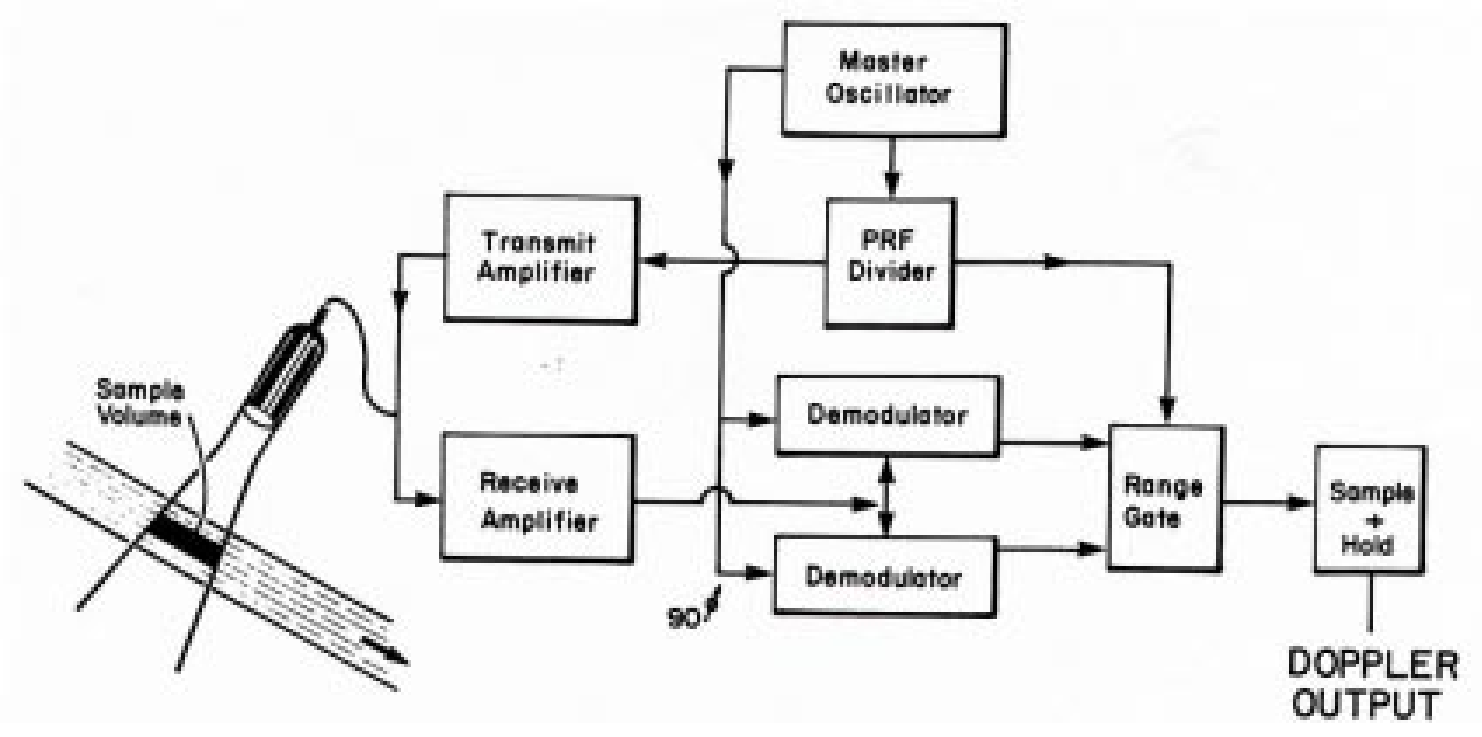

Figure 2.5: Block Diagram of a pulsed-wave Doppler instrument. [Wey94]

The basic pulsed Doppler system has a master oscillator that generates a sinusoidal waveform at a selected clock frequency (say $10 \mathrm{MHz}$.). At specified intervals (determined by a user configured PRF, pulse repetition frequency, divider), a few cycles from the master oscillator are passed through a transmission gate and amplified to shock 
excite the transducer. The shock produces a pulse that moves at the speed of the sound. The frequency at which the transmission gate opens determines the number of pulses transmitted per unit time and hence defines the PRF of the instrument.

A pulsed Doppler system uses range-gated detection to apply the principle of pulse-echo range measurement, for the selection of Doppler frequency shift signals from moving targets. There are two essential steps in the process. First, the phase of the received echo signals are compared with that of a reference signal equal in frequency and having a fixed relationship in time to the transmitted ultrasonic pulses. The phase relationship between these two signals is constant in the case of stationary reflectors but is shifted by the Doppler effect with echoes from moving reflectors. The phase component contains the Doppler shift data.

The second step involves the range gating of the output from the Doppler detector so the Doppler signals corresponding to the chosen depth along the ultrasonic beam can be selected according to the pulse-echo delay time. The range-gated samples are then fed to a sample and hold circuit, so that the output from the system consists of the Doppler signal sequentially updated by each pulse.

The maximum Doppler shift frequency that can be detected unambiguously by a pulsed Doppler system is set by the Nyquist limit wherein the sampling rate should exceed twice the highest frequency component in the signal. The maximum pulse repetition frequency is limited by this time delay: following the transmission of an ultrasonic pulse, the echo from the deepest structure has to be detected before the next pulse is transmitted.

When the Nyquist limit is exceeded, aliasing occurs leading to improper Doppler shift information. Higher pulse repetition frequencies permit higher Doppler shifts to be detected but also increase the chance of a range ambiguity wherein the echo from more than one ultrasonic pulse transmitted is received at the same time. 
The sound pulse transmitted by any pulsed Doppler instrument has dimensions of both length and width. The Doppler sample volume is determined by the width of the beam, the receiver gate's length and the emitted pulse's length. The pulse width defines the beam width at any depth. The receiver gate selects one sample volume from which returning Doppler-shifted echoes are accepted. The gate has some length (depth range) over which it permits reception. The shorter gate length improves the signal to noise ratio and the quality of the spectral trace. Longer pulses provide better frequency resolution and an improved peak/average power ratio but at a lower range resolution.

\section{Comparison}

Continuous wave systems provide motion and flow information without depth selection capability. Pulsed Doppler systems provide the ability to select the depth from which the Doppler information is received. A CW Doppler instrument detects the complete Doppler shift frequency, whereas, the pulsed system detects samples of it. Due to large sample volume, CW Doppler system can give complicated and confusing presentation if reflectors with different flows are encountered. It is impossible to define the origin of an individual signal in the presence of multiple vessels or jets. Pulse Doppler system solves this problem by detecting motion or flow at selected distance or depths with relatively small sample volume.

The advantages of CW are that it has narrowest possible frequency bandwidth and that it has no practical frequency limitations. Continuous wave provides optimal signal to noise discrimination because the signal sums over time whereas random noise cancels. The disadvantage in pulse wave system is if the flow rates become so high that the Doppler shift exceeds one half that the pulse repetition frequency then aliasing occurs. There is no Nyquist limit in CW.

Since the CW Doppler fails to provide range resolution, and the range velocity product limitation of the conventional pulsed Doppler is exceeded in many clinically 
important flow states, a variety of instruments have been developed that attempt to increase velocity resolution while maintaining range discrimination. These instruments fall under two general categories: (1) Pulsed systems that operate at PRF's above the limitation specified by the range velocity product (HiPRF and noise Doppler), and (2) $\mathrm{CW}$ systems into which the relative time of transmission of each output wavelength is encoded onto the carrier, so that when an echo carrying a specific piece of code is detected, the time of flight of the pulse can be determined and the depth of the target defined (FM and pseudo-random binary sequencing Doppler).

\section{Multigate Doppler}

Multigate Doppler devices produce a signal that can be related to the peak velocity within the beam or to flow at specific points along the beam. When multiple targets are present, such as the red blood cells in flowing blood, their spatial location and distribution may also be of interest. This type of information can be obtained by Doppler flow mapping. Doppler flow mapping uses the recorded presence of flow at a series of specific ranges to map the spatial distribution of flow within a specified sampling area. Multigate Doppler systems sample electronically from multiple range gates positioned at equal intervals along the beam path. The velocity distribution is then determined by processing the signal received at each range. If the beam transects a large region of interest, the flow signal at each range can be defined and the velocity output observed continuously. Another approach is to output the data in a binary format indicating flow as the presence of a signal, and no flow as its absence. In this format, the transducer can be swept across a two-dimensional region of a vessel producing a map of the spatial distribution flow. 


\subsection{Doppler signal processing}

This section will look into the details of how the Doppler frequency shift signal is analyzed to extract information related to blood flow characteristics. When the beam of ultrasound is backscattered by a group of blood cells moving with a certain velocity, the information about the velocity is embedded in the Doppler frequency shift, while the amplitude of that frequency component is representative of the number of cells moving at that velocity which causes the backscattering. Hence, the frequency spectrum (amplitudefrequency relation) is of primary interest while the time-domain depiction is difficult to interpret.

The frequency spectrum of the shift signal when there is only one group of cells or tissue moving in the path of the ultrasonic beam will contain a single frequency component, with amplitude proportional to the velocity of the moving tissues. If the beam is incident upon two groups of cells, each group moving at a different velocity, the Doppler shift signal will contain two frequency components with two different velocities. In reality the ultrasonic beam intercepts a large number of cells, all moving at different velocities within a certain range. Therefore they each contribute a different frequency component to the Doppler shift signal. The resultant frequency spectrum will have many components such that it will be a continuous band of frequencies within a certain range. This plot of frequency versus amplitude is called the spectral distribution of the Doppler shift signal. The area under this curve between any two-frequency limits is proportional to the number of cells traveling between the corresponding velocity limits. The frequency $f_{p}$ corresponds to the frequency component having the largest amplitude in the Doppler frequency shift signal. 


\subsubsection{Noise}

Before going further in the signal analysis of Doppler shift signals let us look at the noise content of the Doppler shift signals. The Doppler shifts are continually changing over the cardiac cycle, that is, over a beat. Factors like position of transducer, large sample volume, Doppler angle, inspiration, expiration, scattering and reflection from moving solid structures of heart, vessel wall and valve leaflets contribute to the noise in the primary Doppler shift spectrum.

The other types of noise encountered are channel noise and correlation or echo noise. The strong Doppler shift echoes originate from slow moving walls of the vessels. These high intensity low frequency echoes or clutter, which overwhelm the weaker echoes from the blood, are generally filtered out during recording the signal using high pass wall filter. The Doppler signal is generally considered as Gaussian random process. The noise in ultrasonic systems is governed by additive Gaussian noise resulting from transducer and amplifier. [Ang85]

\subsubsection{Spectral Analysis}

Any analysis of the Doppler shift signal is performed using the frequency-domain representation. Of the several forms of spectral analysis the two most widely used are:

(1) Approximate spectral analysis using time-interval histogram.

(2) Fourier analyses resulting in precise information about the amplitudes and frequencies of the different components that make up the Doppler shift signal.

Of these methods the second method is accurate and is widely used. With the advent of sophisticated electronic instrumentation, true frequency analyses (i.e., Fourier analysis, 
particularly fast Fourier transformation algorithm) are gaining popularity. This method of Doppler frequency shift signal analysis is discussed.

Fourier analysis is a plot of the amplitudes of the different frequency components that make up a signal. A plot indicating the relative time instants within a cycle when the peaks of the different frequency components occur is called a phase plot or phase spectrum. The phase spectrum is usually much less important than the amplitude spectrum because the latter contains all the information related to the distribution of energy at different frequencies within the summated signal. There are two methods for calculation of Fourier spectrum, band pass filtering and fast Fourier transforms.

\section{Band-pass filtering}

If a Doppler shift signal were passed through a band-pass filter, the resultant output would contain only those frequency components within the frequency shift signals that fall within the pass band. If the frequency shift signal is passed through many bandpass filters, the resultant outputs represent the energy content of the input signals falling within the respective pass-bands. If the pass-bands are arranged in a graded manner to extend from the lowest to the highest frequencies of interest, the outputs of the band-pass filters when placed side by side and plotted: represent the amplitude spectrum of the input signal. The primary disadvantage of this method is the large number of band-pass filters required to obtain adequate resolution along with the frequency axis.

\section{Fast Fourier Transforms}

The fast Fourier transform is an efficient algorithm for computing the frequency spectrum of a signal from its digitized values. The availability of computers in the form of microprocessor chips that can be incorporated into the Doppler instrumentation now permits the design of stand-alone instruments that contain not only 
analog-to-digital converter chips but also chips that implement fast Fourier transforms. The Doppler frequency shift signal, which is obtained by comparing the transmitted and received ultrasound frequencies, is first sampled and digitized by the analog-to-digital converter. This converter feeds the numeric data produced directly into the FFT processor. The result of this is fed into a display processor, which converts these numbers into a graphic display on screen, or into a hard copy.

\subsection{Cardiac Cycle and Cardiac Output}

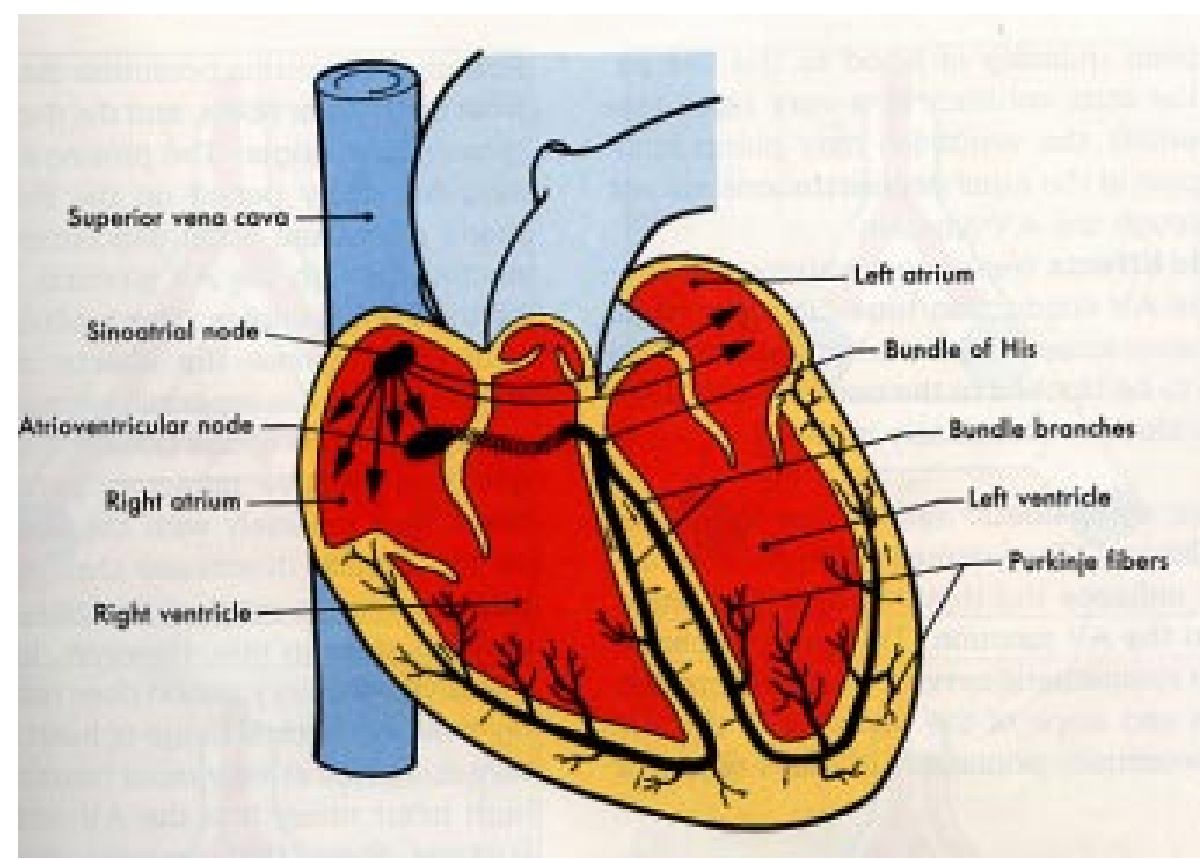

Figure 2.6: The heart and its chambers. [Ber90]

The heart comprises of four muscular chambers namely the left and right atrium and ventricles. [Ber90] The atria pump blood to ventricles and the ventricles pump blood to body. Each chamber ejects blood through a valved outflow aperture. The cardiac cycle, described from perspective of ventricle, comprises four phases of unequal duration, with events on the right and left sides being almost synchronous. In the filling phase of the 
ventricle, the heart is in diastole (minimum volume of blood); the arterial outlet valves (to the ventricle) are closed and the atrioventricular inlet valves (to the body) are open, allowing passive filling of the ventricles. This is boosted by atrial contraction giving the maximum volume of blood in the ventricle. In the isovolumetric contraction phase of the ventricle the onset of ventricular contraction or systole raises ventricular pressure, which closes the atriventricular valves. Pressure then rises rapidly until the opening of the arterial outlet valves and ejection of two thirds of the ventricular blood. As ejection rate wanes, pressure falls until the outlet valves are closed by a slight back-flow. The final phase is the isovolumetric relaxation phase where the ventricular pressure falls rapidly, until it is less than arterial pressure, at which point the atroiventricular valves open and the filling phase commences. Since electrical impulses stimulate mechanical contraction, the cardiac mechanical cycle can be synchronized with the electrocardiogram signal.

An electrocardiograph (ECG, Figure 2.7) [Ber90] records the electrical activity of the heart. An ECG signal reflects the temporal changes in the measurements of the heart's electrical activity by attaching electrodes to specific points on a person's chest, arms, and legs. An ECG cycle is represented by the P wave, the QRS complex, the T wave, and the baseline that follows until another $\mathrm{P}$ wave appears.

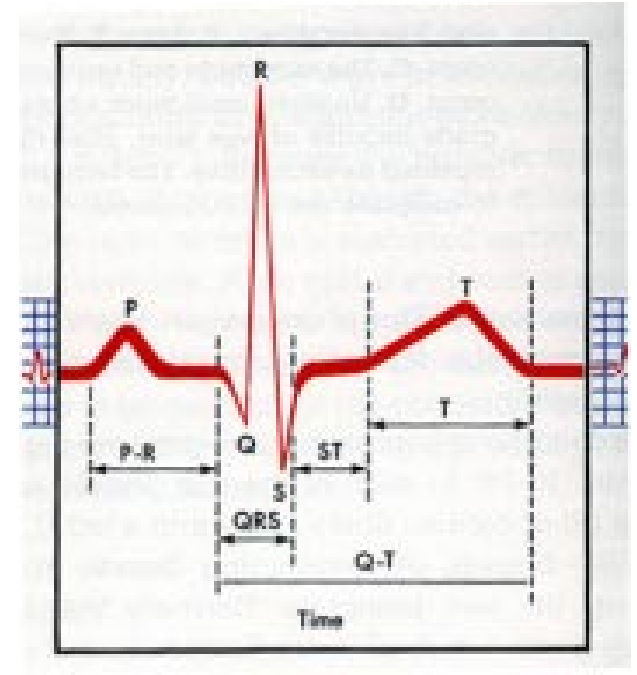

Figure 2.7: The ECG signal. [Ber90] 
The P-R interval is a measure of time from the beginning of atrial contraction to the beginning of the ventricular contraction. The configuration and amplitude of the QRS complex vary considerably. It represents the passage of the electrical activity in the ventricles. The $\mathrm{T}$ wave represents the reset of the ventricles. The Q-T interval is referred to as the period of 'electrical systole' where as the P-R interval is known as 'diastole'. The entire duration of this cycle varies individually according to body structure and activity. Normally it varies from .5 seconds to .9 seconds.

Cardiac output is the volume of blood pumped by the heart per unit time. [Bow91] The unit of measurement is generally expressed in liters per minute. The heart operates as a pulsatile pump that ejects a bolus of blood, known as stroke volume, with each cycle of contraction of heart muscles. Cardiac output (CO) is the product of stroke volume (SV) and heart rate (HR),

$$
C O=S V \times H R
$$

where $\mathrm{CO}=$ cardiac output

$\mathrm{SV}=$ stroke volume

$\mathrm{HR}=$ heart rate

The cardiac output depends upon a complex set of interrelated physiological variables like the volume of blood in the heart, the downstream resistance to ejecting blood from the heart, and the contractility and efficiency of the heart muscle. The metabolic requirements of the body also influences the cardiac output. A complex network of nerves called the autonomous nervous system that regulates the activity of the cardiovascular system facilitates the integration of the heart and metabolic demand. Cardiac output not only determines the functional state of the heart but also the response of the entire circulatory system to acute and chronic diseases and the impact of the therapeutic interventions. Basal cardiac output is related to body size and varies approximately four to seven liter per minute in adults. 


\subsubsection{Measuring Cardiac Output [Bow91]}

Over the years, different methods were adopted for measuring cardiac output in humans. Adolph Fick described one of the first techniques in 1870. It was postulated that the cardiac output could be calculated from the difference in oxygen content between the mixed venous (pulmonary artery) and arterial blood and the total body oxygen consumption. This method is considered the gold standard for measuring cardiac output. Severe limitations exist for the use of this technique in the clinical settings. This method is most accurate when the cardiac output is normal or reduced but is ineffective at adverse physiological conditions.

Stewart first introduced the indicator dilution technique also known as indirect Fick method for determination of cardiac output in 1897 that was later modified by Hamilton in 1932. Measurable indicators like inert dyes, gases, hypertonic saline and cold saline or dextrose solution of known concentration are injected into the circulation. The indicator mixes with blood and is thereby diluted. The extent of dilution, determined by measuring the concentration, is inversely proportional to the blood flow. In the dye dilution method, a bolus of dye, usually indocyanine green is injected rapidly into the venous circulation near the right atrium and the downstream concentration of the dye is measured from a peripheral artery. A blood sample is withdrawn continuously from the artery and passed through a densitometer that determines the concentration spectrophotometry. A chart recorder produces a dye concentration versus time curve. The area under the curve is inversely proportional to cardiac output. Like the Fick technique, dye dilution is also not suitable for routine clinical use. Calibration of the equipment is difficult and the repeated determinations of cardiac output are limited by the accumulation of dye in the circulation.

With the introduction of the pulmonary artery catheter, the thermodilution technique for cardiac output measurement became famous. It is similar to the indicator technique in some ways. The indicator for this technique is a cold fluid, which is cooler 
than the subjects' blood, usually saline solution either iced or at room temperature. The cold liquid is injected through the pulmonary artery catheter, into the right atrium, where it mixes with the venous blood and causes the blood to cool slightly. The cooled blood is ejected by the right ventricle into the pulmonary artery, where it passes by a thermistor near the tip of the pulmonary artery catheter. The thermistor measures the change in blood temperature as the cooled blood travels past on the way to the lungs. The extent of cooling is inversely proportional to the cardiac output. The thermodilution technique has undergone many modifications and is very popular technique. It has a few shortcomings also. Lot of errors are found in the cardiac output reading due to the intravenous administration of fluids. This error occurs because the rapid administration of intravenous fluid causes cooling of the thermistors, which combines with the cooling caused by the injectate and results in smaller calculated cardiac output. This type of error can happen easily in the critical care environment, especially the operating room, where the fluids are commonly infused rapidly. This error can also occur because of the unstable nature of baseline pulmonary artery temperature.

\subsubsection{Cardiac output using ultrasound}

All the classical techniques for measuring cardiac output are somewhat unsuitable for continuous time measurements in clinical conditions, motivating the need for a noninvasive measuring technique. Ultrasound can estimate cardiac output by measuring the velocity of blood flow in the ascending aorta by the application of the Doppler principle. Ideally, if the blood is flowing at a single velocity and the cross sectional area of the aorta is measured, then the blood flow rate can be calculated as follows.

$$
\begin{aligned}
\text { Blood Flow } & =\text { velocity }(\mathrm{cm} / \mathrm{sec}) * \text { area }\left(\mathrm{cm}^{2}\right) \\
& =\text { Liter/minute }
\end{aligned}
$$


The blood flow in the ascending aorta is identical to the difference of cardiac output and the flow in the coronary arteries. Ultrasound cardiac output devices measure the stroke volume by integrating blood velocity during the cardiac systole.

$$
S V=C S A \times \int{ }^{V E T} V(t) \partial t
$$

Where SV = stroke volume

$\mathrm{CSA}=$ cross sectional area of the aorta

VET $=$ ventricular ejection time

$\mathrm{V}=$ blood velocity

Stroke volume is then multiplied by heart rate to give cardiac output. This is a very reliable technique that can be used for uninterrupted beat-to-beat measurement of cardiac output when compared to the other techniques. The difficulty is that at any single point in time at a particular cross-section of the artery, the blood is not flowing at a single velocity. This necessitates further analysis to determine a good estimate of velocity. 


\section{CHAPTER 3: Different Approaches}




\section{DIFFERENT APPROACHES}

In the recent past, many methods of Doppler signal analysis have been proposed. The focus has been on automated, real-time spectral analysis techniques that require minimum human interaction. Fourier or Z-transform based analysis is done for the extraction of suitable parameters. Most of the methods were formulated for the common goal of finding the cardiac output from the Doppler shift signals. Almost all the investigators compared their output of Doppler measurements with parallel estimates of cardiac output obtained by other methods like the thermodilution technique while in some cases of real time methods, synthetic signals were analyzed. The proposed methods have a blend of hardware and software components.

The main aim of these algorithms is to calculate the peak frequency of the Doppler shift signal over a specified time interval. Even though multiple velocities of blood and therefore multiple frequencies in the Doppler shift are present, in order to calculate cardiac output only the peak frequency in the shift signal that has significant power is needed. It was shown in [Eri90] that the mean velocity of blood ejected from the valve of the heart is equal to the maximum velocity of blood farther down in the aorta where the Doppler measurements are taken. The maximum velocity or peak frequency times the cross sectional area of aorta will give instantaneous flow. The integration of the velocity with respect to time gives the stroke volume integral. This when multiplied by cross section area of the aorta gives the instantaneous stroke volume. Stroke volume times the heart rate gives the cardiac output.

Eriksen and Walloe studied the degree of ultrasonic attenuation in the tissues and proposed an improvement to the existing ultrasound Doppler methods for measuring cardiac output. [Eri90] According to them the quality of the signals located near the aortic valves is inferior due to strong Doppler shifted sound reflected from the walls of the heart and the valve leaflets. The signal strength tends to drop significantly in the 
inspiratory phase compared to the expiratory phase due to the above-mentioned motion near the valves. This method predicts that the blood velocity remains unaltered few centimeters above the aortic valve and that a sampling volume at that position would give a stronger signal for spectral analysis. The analysis was performed on $10 \mathrm{~ms}$ of signal at a time. It was observed that there is no significant change in the velocity profile during the $10 \mathrm{~ms}$ window of the flow. The Doppler shifted echoes due to the slow moving objects were filtered out using a high pass filter with the threshold velocity typically around $0.2 \mathrm{~ms}^{-1}$. This study is the basis for which cardiac output can be calculated from the Doppler signal.

D'Alessio proposed an algorithm based on the statistical characteristics of fast Fourier transform (FFT) spectral estimators. [DA185] This method is based on reliable discrimination between signal and noise. It can be used to estimate maximum frequency and spectral broadening independent of human intervention and the gain of spectral analyzers. The Doppler signal is modeled as a zero mean Gaussian process with varying amplitude and bandwidth during the cardiac cycle. The discrimination between signal and noise is a two-step process based on the specificity and sensitivity. Specificity is defined as the probability that a spectral bin can be correctly recognized as due only to noise, whereas the sensitivity is the probability of detecting a spectral bin when due to signal and noise. The rationale of the algorithm is determination of two thresholds that is determined to attain a prefixed value of specificity while maximizing sensitivity. Using this threshold the signal to noise ratio of the signal is estimated. The peak frequency is then evaluated from the power spectral density based on the isolation of signal. Doppler signals recorded under normal conditions were recorded and then different levels of white noise were added to the signal. Experimental results of this method showed that the method was satisfactory for the signals with synthesized noise superimposed on it.

Heringa A., Daniels et al., proposed a method [Her88] for reliable automatic extraction of parameters from cardiac Doppler signals more reliably without human interaction. They hypothesized that the noise spectrum can be estimated from Doppler spectrum by taking the average level over the interval where a blood flow signal is not 
present. See figure 3.1 and figure 3.2 for an example of spectra during noise and during blood flow. If independence between the signal and noise is assumed and their respective powers is considered additive, noise levels can be estimated and subtracted. The Doppler shift data was collected from a Honeywell Ultra-Imager at a sampling frequency of 13 $\mathrm{kHz}$. Doppler signals were analyzed in intervals of $5 \mathrm{~ms}$ to $20 \mathrm{~ms}$ using spectral analysis with a Hanning window

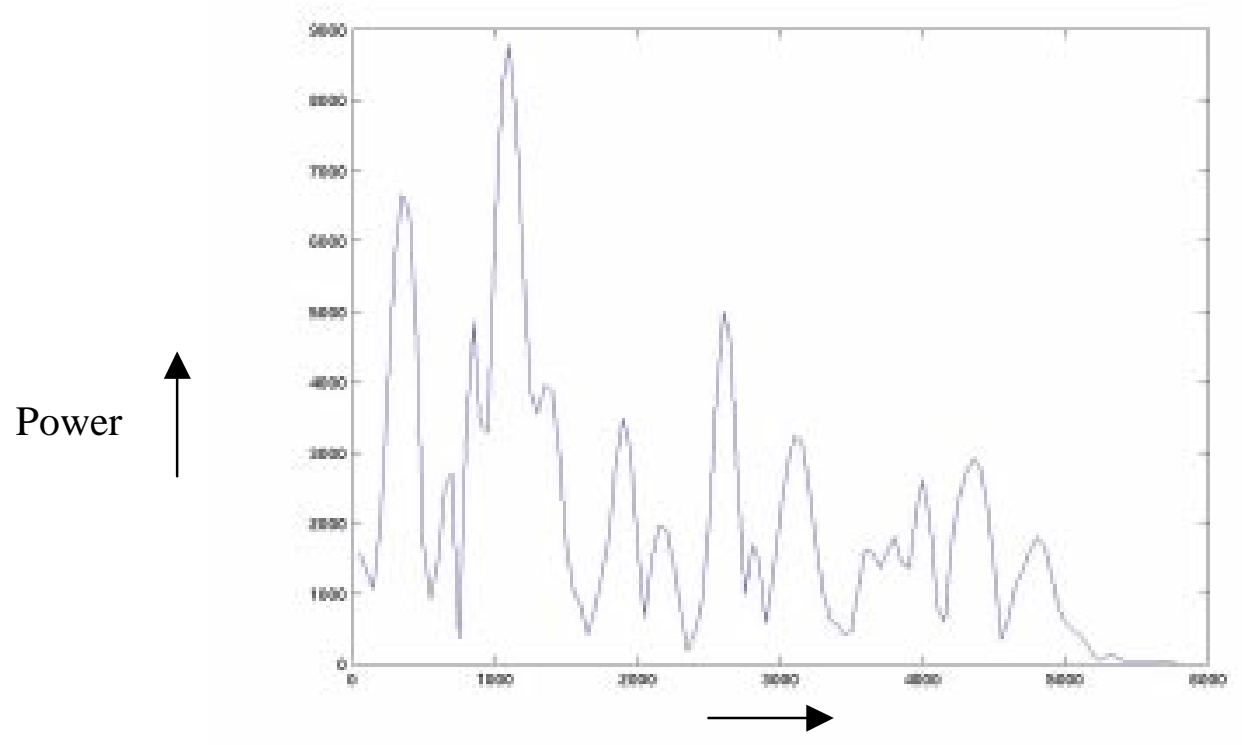

Frequency

Figure 3.1: Fast Fourier transform of a noisy Doppler shift signal (when there is no blood flow). Since this portion of the Doppler shift signal only contains noise, no peak frequency should be calculated.

For five seconds, the power spectrum was converted to a distribution for each frequency bin. It is assumed that flow is present during the maximum 66 percent of the time in the 5-second signal. The lower 33 percent is considered to be the time when there is no flow and hence noise or the lower 33 percent of the frequency bin distribution. A zero mean level was obtained by subtracting 1.66 times the noise part of the signal. The multiplicative factor 1.66 was derived from the 33 percent noise signal. Each spectrum is 
smoothened to make the estimates of modal and maximum frequency less variable. Maximal value of the median curve, the interquartile range of that moment and the maximal acceleration during systole were chosen as the elementary characteristics per heartbeat and could be used to estimate cardiac output.

This method effectively extracted parameters from the cardiac ultrasound Doppler spectrograms automatically by using the knowledge of the behaviors of the Doppler signal. Parameters are extracted and reproduced in a reliable way by subtracting the background noise and smoothing the raw signal. These parameters can be used in characterization of the Doppler data. The algorithm is incorporated into the hybrid algorithm designed for this thesis.

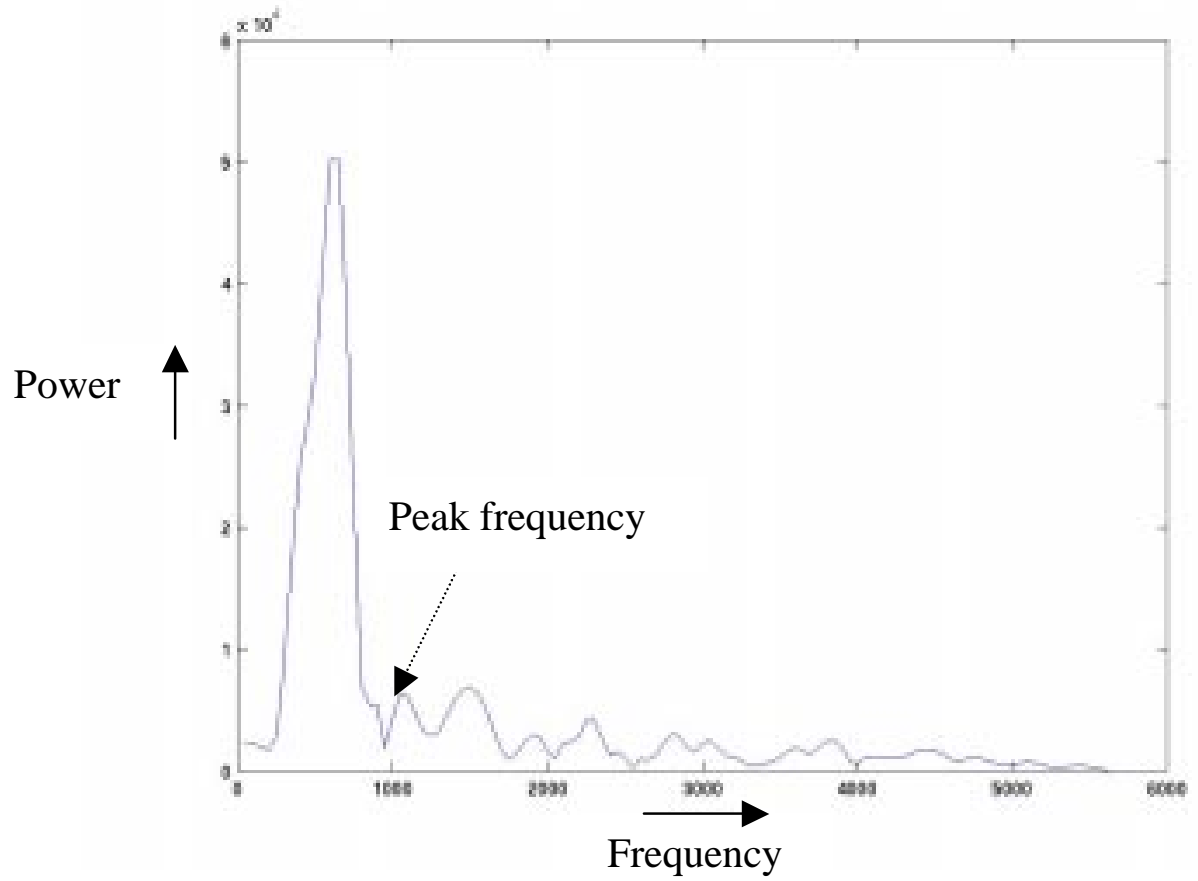

Figure 3.2: Fast Fourier transform of a Doppler shift signal (when there is blood flow). The peak frequency is selected where power goes to "zero". 
Larry Y. L. Mo et al [Mo88] did a comparison of the performance of four digital maximum frequency estimators for Doppler ultrasound signal. The methods investigated are percentile method, D'Alessio's threshold crossing method, a modified threshold crossing method and a new hybrid method. Performance was evaluated under a variety of signal to noise (SNR) ratio and signal bandwidth conditions. A general approach in the paper to the estimation of maximum frequency relies on the derivation of power spectrum of signal and noise by taking the fast Fourier transforms (FFT) of the time domain signal.

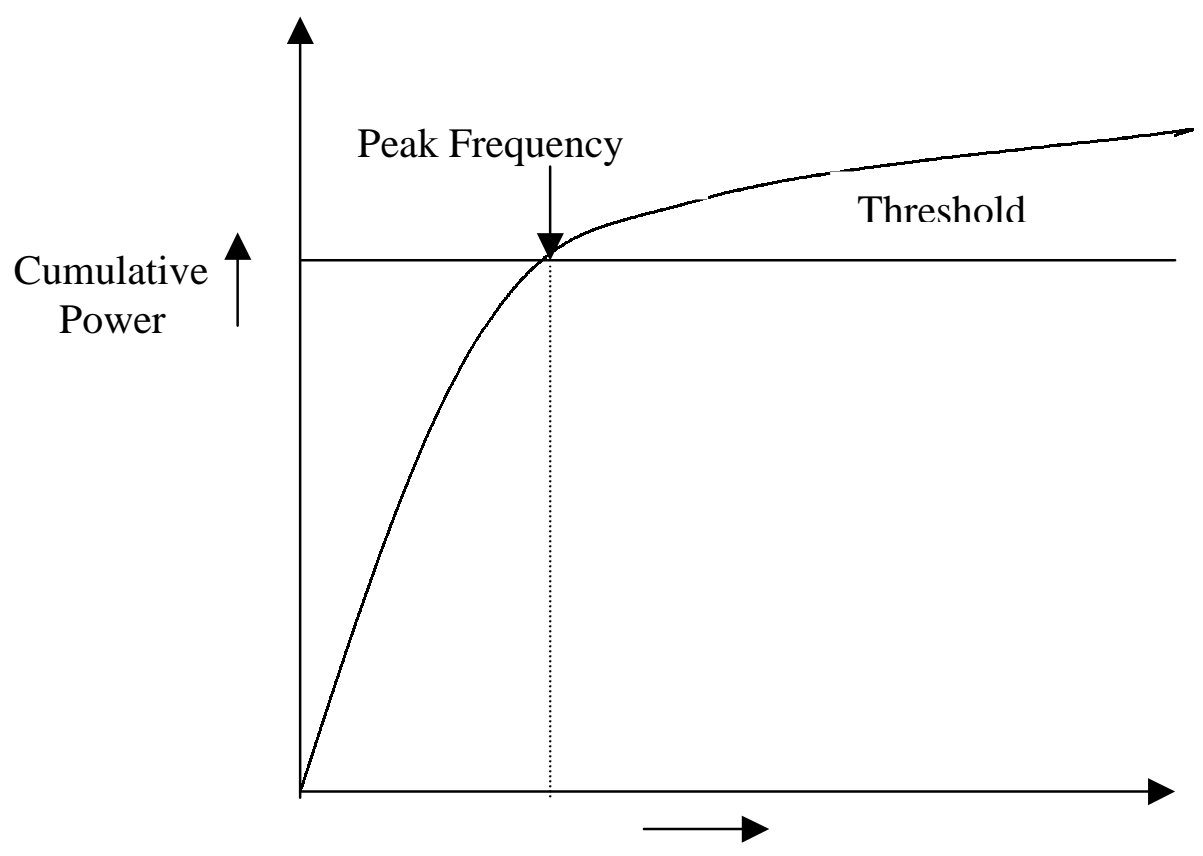

Frequency in $\mathrm{kHz}$.

Figure 3.3: Peak frequency location in the percentile method. The $x$-axis is frequency; $y-$ axis is cumulative power up to that frequency. Frequencies above the "knee" is portion where only power from noise is added to the cumulative power. This point is the peak frequency where signal is present.

Maximum frequency can be estimated from the integrated Doppler power spectrum as shown in the figure 3.3. Each frequency on the plot is the summation of power from zero to that frequency. The curve is very steep at lower frequency where 
signal is present and reaches a steady state at higher frequencies. The transition from the signal region at the lower frequencies to the purely noise region of the higher frequencies is characterized by a distinct knee. This knee is defined as the maximum frequency. The four methods describe the different ways to locate this knee.

In the percentile method, the maximum frequency is picked at the intersection of the curve and a threshold, which is based on a percentage of the maximum power. Typically, threshold is $90 \%$ to $98 \%$ of maximum power depending on the SNR of the signal. In the threshold crossing method, D'Alessio's algorithm is derived from theoretical consideration based on the chi-square statistic of the white noise spectrum. The theory assumes the usage of rectangular window data for spectral estimate. In this method, spectral samples in successive frequency bins are compared to a threshold level, starting from the noise end. If in a sequence of, say ' $r$ ', successive bins there are at least $\mathrm{m}$ bins that exceed the threshold, the peak frequency is taken to be the highest bin frequency in that sequence.

In the modified threshold crossing method, a non-rectangular window like a Hanning data window is used. The estimation of the peak frequency is similar to that of D'Alessio's method. When the SNR is low, in both these methods, noise may cause the threshold to be crossed at a much higher frequency than the actual frequency. This problem is difficult to overcome in these methods.

The hybrid method combines features of both the percentile method and the threshold crossing method. In this method, the knee of the curve is located using a straight line with a slope, $N_{o}$, the noise power density, which is typically estimated by averaging over the tail end of the power density spectrum.

It was found [Mo88] that the D'Alessio's method performed well at lower SNR only. At higher frequencies, there is higher spectral leakage due to the rectangular nature of window. The modified threshold crossing and the hybrid methods show consistent performance over wide range of the test conditions. In these four methods, the accuracy 
in calculation of noise power density is critical. These methods have similar computational complexities.

Among the various techniques that estimate the peak frequency of Doppler shift signal, Rickey D.W. and Fenster A. [Ric96] reported the performance of an advanced real-time automated peak-frequency tracing algorithm implemented by Routh et al., [Rou94] on a spectral Doppler instrument. Comparison of measurements made by this automated algorithm to known quantities was performed. Errors introduced when tracing the waveform were taken into account during the analysis whereas errors introduced by variations in sample volume location, patient physiology, and transducer selections were left out.

The technique of determination of peak frequency from the algorithm is based on calculation of a threshold which is derived from the average signal to noise ratio over a cardiac cycle. A convenient block of signal is taken for the determination of the spectrum of the signal. A signal block of $10 \mathrm{~ms}$ is taken because it is asserted that the velocity of the blood in the aorta is very near to constant over a period of $10 \mathrm{~ms}$. The velocity profile is rectangular over $10 \mathrm{~ms}$. The algorithm selects the highest frequency in the spectrum whose magnitude is equal to the threshold level. The threshold is initially set to $-3 \mathrm{~dB}$ of the maximum amplitude of the first spectrum. This is the peak frequency for each block of signal.

The threshold is updated once every cardiac cycle. This is accomplished in an iterative manner. A new threshold for the next cardiac cycle is calculated using the current threshold and the spectra in the current cardiac cycle. The subsequent threshold is determined from the ratio of average of signal and noise over the cardiac cycle. The subsequent threshold is the signal intensity peak (in $\mathrm{dB}$ ) minus the $\mathrm{SN}$ ratio.

The first step in determination of peak velocity is to find the peak intensity of each successive spectral line and compare its value with the average noise value of the entire previous cardiac cycle. If the peak intensity is greater then the spectrum is valid 
and the maximum frequency in the spectrum where the threshold meets can be considered as the peak frequency for that spectrum. All the other data points that are above the threshold are considered as SIGNAL and the points below the threshold are considered as NOISE. For each spectra the average of signal and noise is calculated. This procedure is continued over the cardiac cycle and the average of all such SIGNAL and NOISE is calculated. The ratio of this signal to noise gives the threshold for the next cardiac cycle. An additional step is the determination of a 10-millisecond segment of signal. Once the peak frequencies over the cardiac cycle are noted, cardiac output can be calculated.

$\mathrm{SN}$ ratio $=$ Average signal $/$ Average noise 


\section{CHAPTER 4: Procedure}




\section{PROCEDURE}

The Doppler shift signals used for the processing were recorded in simulated environment using Biosound Inc's Genesis II [www1] annular phased array duplex ultrasound measurement instrument. The same instrument was used to measure the cross sectional area of the aorta by Doppler imaging. The Doppler shift signal and the electrocardiogram signals were measured simultaneously in time and were stored using a digital tape recorder at a frequency of $12000 \mathrm{~Hz}$. The ultrasound instrument takes into account the Doppler shift angle before giving the shift signals. Hence the Doppler angle is not considered in calculation of the peak velocity from the Doppler shift signals. The recording of these signals follows a protocol. The subject whose signals are recorded were supine during the first part of the recording. After few minutes the operational tilt starts. The Doppler shifts recorded before and during the tilt test forms the data set for the analysis.

The data stored on the digital audiotapes were viewed on a personal computer using a digital audio data recorder, RD-135T, manufactured by TEAC Inc. [www2] The data recorder is connected to the computer through the QuikVu interface board. The QuikVu software enables the viewing of the digitized signals in chart mode without delay in time. It has many in built features that allow portions of data in time domain to be saved in MATLAB format. Two channels of data were simultaneously stored in the MATLAB ASCII file format. The first channel has the Doppler shift signal while the second channel has electrocardiogram signal. The second channel is necessary for aligning the Doppler shift processing beat to beat. The data was sampled at $12 \mathrm{kHz}$. 

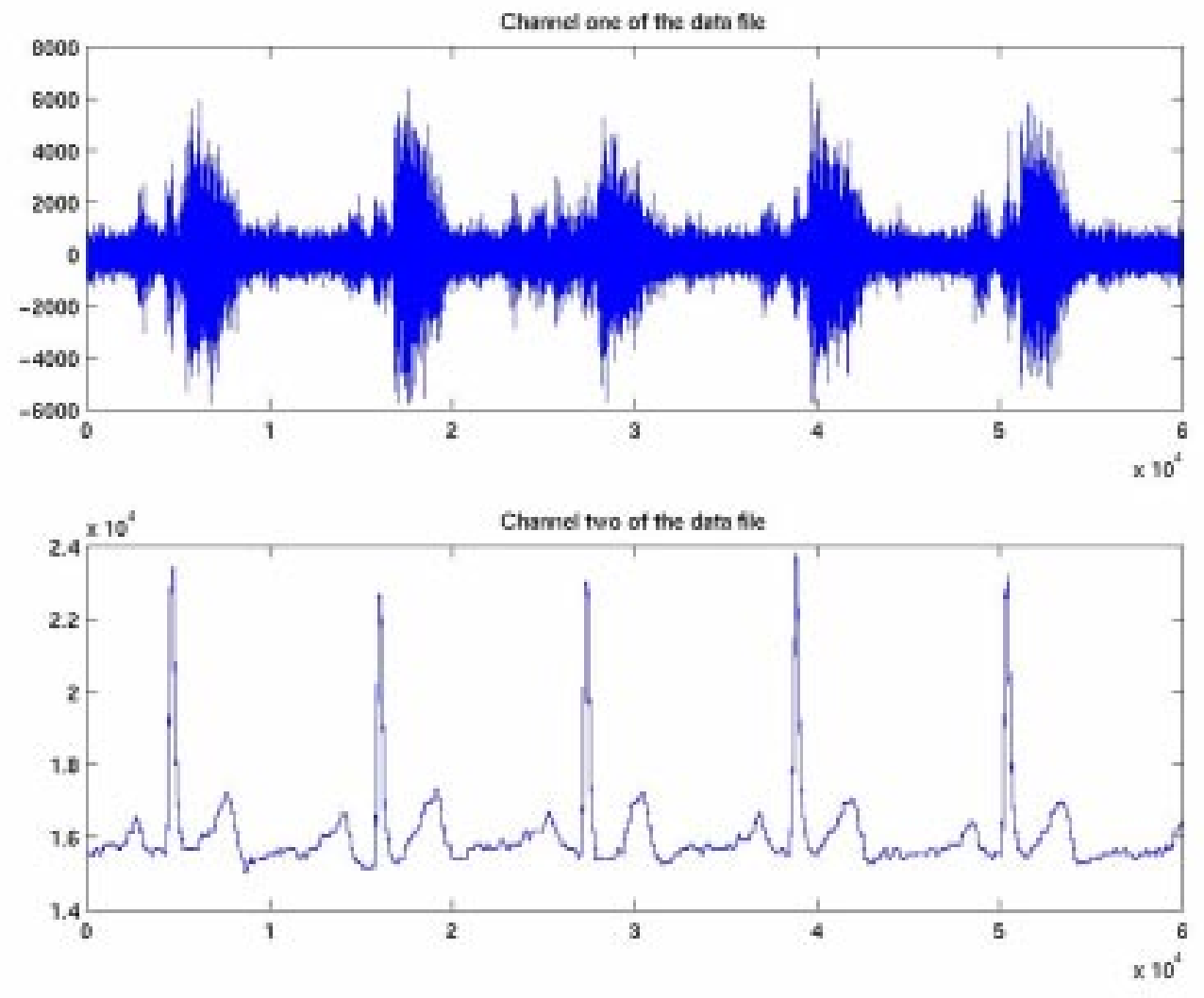

Figure 4.1: Typical data over a period of 5 seconds. The top graph is the Doppler shift signal and the bottom is the electrocardiogram. The $\mathrm{x}$-axis is sample points and $\mathrm{y}$-axis is relative amplitude.

Along with the signal, there is data of one-minute duration containing the instantaneous heart rate and the manually calculated stroke volume values. This data set is the reference value used in validating the results of this thesis. The output of the ultrasound Doppler measuring instrument is displayed on a screen. The gray scale display of FFT is manually read and the peak frequency is noted. The envelope of the peak velocity versus time is traced and thus the stroke volume integral is found out. The cross sectional area of the aorta is got by using Doppler echo imaging system. The product of the cross sectional area of aorta and the stroke volume integral gives the stroke volume. 


\subsection{SNR algorithm}

In the first phase of project, an algorithm was written based on the Routh's algorithm [Ric95] implemented on Doppler instrument described in the last chapter. The basic principle of this method is to calculate a threshold value $\left(T_{v}\right)$ that selects the highest frequency from a Doppler intensity spectrum. The frequency at which the intensity of the spectrum is equal to that of the threshold value is considered as the peak frequency as shown in the figure. This process is continued for each spectrum beat to beat across the cardiac cycle.

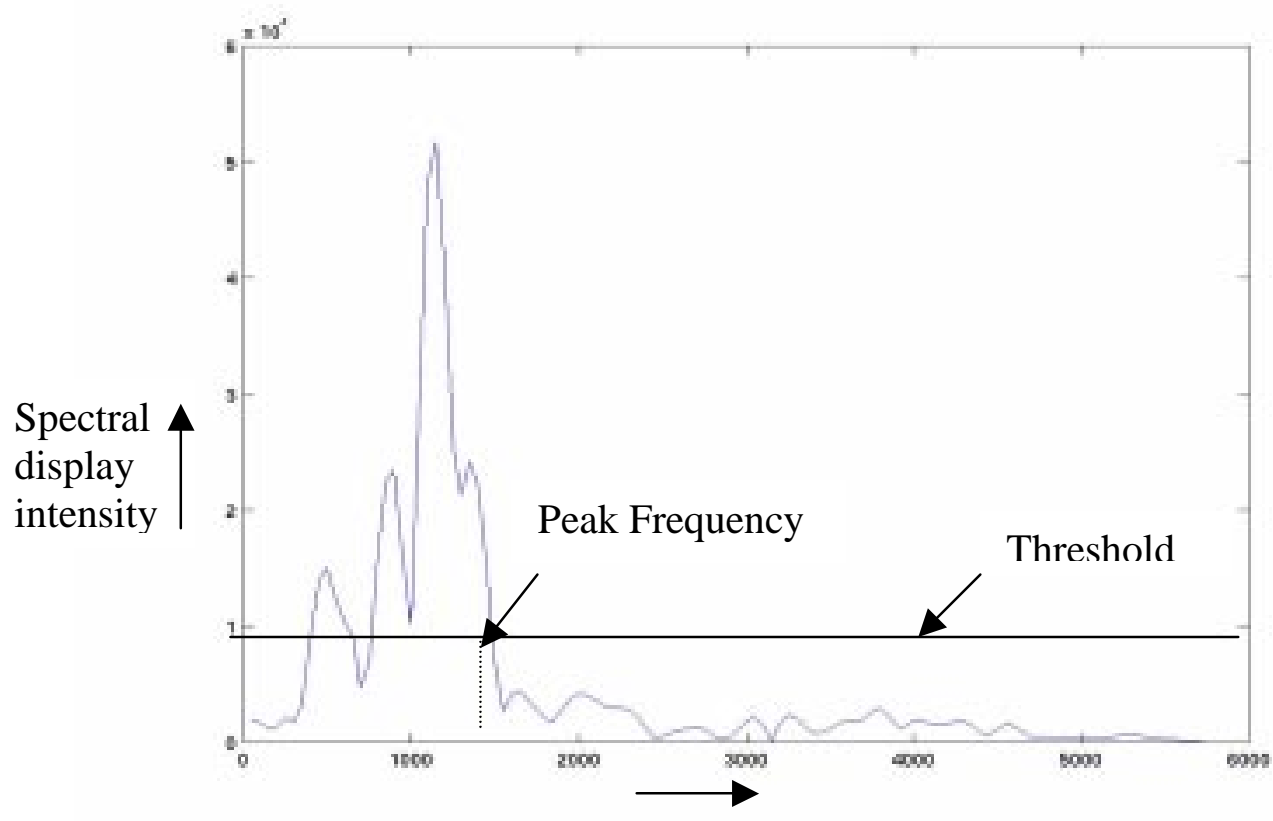

Frequency in $\mathrm{Hz}$

Figure 4.2: Basic concept of automated peak-frequency algorithm. The peak frequency is defined as the highest frequency of the spectrum where significant portion of power is present. 
In this algorithm, the electrocardiogram (ECG) signal was fed to a trigger program that gave the sample points at which the ' $r$ ' wave of a typical ECG signal exists. These trigger points are collected to run the program beat to beat. A portion of 10 milliseconds duration of the Doppler shift signal is taken at a time for analysis, which accounts for 120 samples of signal. A signal block of $10 \mathrm{~ms}$ was used because it is asserted that the velocity of the blood in the aorta is very near to constant over that time period. [Ang85] The Fourier transform of this signal is taken over 240 points. In order to ensure that the peak frequency measurement is true to the signal the threshold is updated every cardiac cycle. This offsets changes in the intensity of the shift signal due to instrument settings like power and gain, motion artifacts, etc. The threshold for the next cardiac cycle depends upon the signal to noise ratio (SNR) of the present cardiac cycle. Hence, the threshold is updated iteratively as shown in the flowchart of the algorithm [Appendix: 2]

Initially, the threshold is set to half the power of the maximum intensity of the Doppler shift spectrum. The intensity is estimated by taking the absolute value of the fast Fourier transform of the signal segment. The subsequent threshold is the ratio of average of signal and noise over the cardiac cycle.

\section{SNR $=$ AVERAGE SIGNAL $/$ AVERAGE NOISE}

The updated threshold is the peak of the maximum intensity (in $\mathrm{dB}$ ) minus the SNR (in $\mathrm{dB}$ ). The first step in determination of peak velocity is to find the peak intensity of each successive spectral line and compare its value with the average noise value of the entire previous cardiac cycle. If the peak intensity is greater then the spectrum is valid and the maximum frequency in the spectrum where the threshold meets can be considered as the peak frequency for that spectrum. All the other data points that are above the threshold are considered as SIGNAL and the points below the threshold are considered as NOISE. For each spectrum the average of signal and noise is calculated. 
This procedure is continued over the cardiac cycle and the average of all such SIGNAL and NOISE is calculated. The ratio of this signal to noise is used to determine the threshold for the next cardiac cycle.

Once the peak frequencies over the cardiac cycle are noted, the peak frequency is converted to peak velocity using the simple Doppler shift equation. Once the peak velocities over the cardiac cycle are noted, a velocity versus time curve is plotted. The area under this curve gives the stroke volume integral. This when multiplied by cross section area of the aorta gives the instantaneous stroke volume. Stroke volume times the heart rate gives the cardiac output

This algorithm [Ric95] uses some ambiguous constants in defining the threshold. According to the proposed algorithm the threshold is given as below iteratively over the cardiac cycles.

$$
\text { Threshold }=\frac{\text { Average_signal }_{\text {Average_noise } \times k}}{\text { Ave }}
$$

Where $k$ is defined as a constant dependent upon the scroll rate of the display of the Doppler ultrasound instrument.

In the estimation of the maximum intensity, it is checked if the maximum intensity is higher than the average noise of the previous cardiac cycle. In order to ascertain this fact the maximum intensity is checked if it lies in the range of $K_{L} *$ average noise and $K_{H}{ }^{*}$ average noise, where $K_{L}$ and $K_{H}$ are defined as ultrasound instrument dependent constants. The algorithm uses the signal to noise ratio of the previous cycle to ascertain the threshold for the next cardiac cycle. This is not a good estimate because there could be a major difference in the power levels of the adjacent beats. The motion artifacts and other noise in a cycle affect the threshold for the next cycle. Since the first threshold is taken as half power of the maximum intensity of the first spectrum, the SNR 
of this portion of the signal could not be the correct SNR. This would affect the peak frequency estimation of the next few cardiac cycles till there is a steady state in the SNR values. This results in loss in the peak frequency information in some of the cardiac cycles at the beginning of the sequence of beat-to-beat analysis.

Another major problem in this method is that the change in the nature of the signal within a beat goes unnoticed in calculating the peak frequency. This could give serious problems in estimation of the peak velocity. This method also considers the displayed intensities depending upon the gray scale intensity for the calculation of the spectral amplitude. Thus, this method is suitable to the integration with the Doppler ultrasound-measuring instrument. It is not an ideal method for the independent real time analysis of Doppler shift signal for the calculation of the cardiac output. This method was abandoned very early in our efforts due to poor results.

\subsection{Hybrid Percentile algorithm}

After study of the algorithm in the last section and review of the literature, a new method for Doppler signal analysis has been developed combining several algorithms and including some new features. The parts of algorithm that were used from the literature include the noise reduction routine described in [Her88] and the percentile method described in [Mo88]. As in the previous attempt, the data used is two-channel signal comprising of Doppler shift signal and ECG signal both recorded simultaneously in time at a sampling frequency of $12 \mathrm{kHz}$.

The implementation of this hybrid algorithm is done using the MATLAB tools. The input file consists of a two-row data file, the first row has Doppler shift signal at $12000 \mathrm{~Hz}$, while the second has ECG signal sampled at the same rate. The Doppler shift signal should have a zero dc magnitude to avoid variations in the total signal plus noise power. 
The algorithm first runs a trigger program [McD90] on the ECG signal. This program takes into consideration the sampling rate and determines the location of the 'QRS' complex of a typical ECG signal, as shown in the figure 4.3.

The trigger program runs as follows. The signal is first passed through a band pass filter. The absolute value of the output of the band pass filter is taken and the incidence of first maximum value of the signal sequence is located. At that position an amplitude level lower than the maximum value is chosen for threshold. This threshold is allowed to reduce exponentially with increment in time until the cardiac cycle is encountered. The sample number of the ECG signal at the point where the exponentially decreasing function meets the rising ' $R$ ' wave is taken as the trigger point of that particular beat. The set of sample numbers collected at all such ' $R$ ' waves form the set of trigger points. Figure 4.4 shows a typical output of the trigger function.

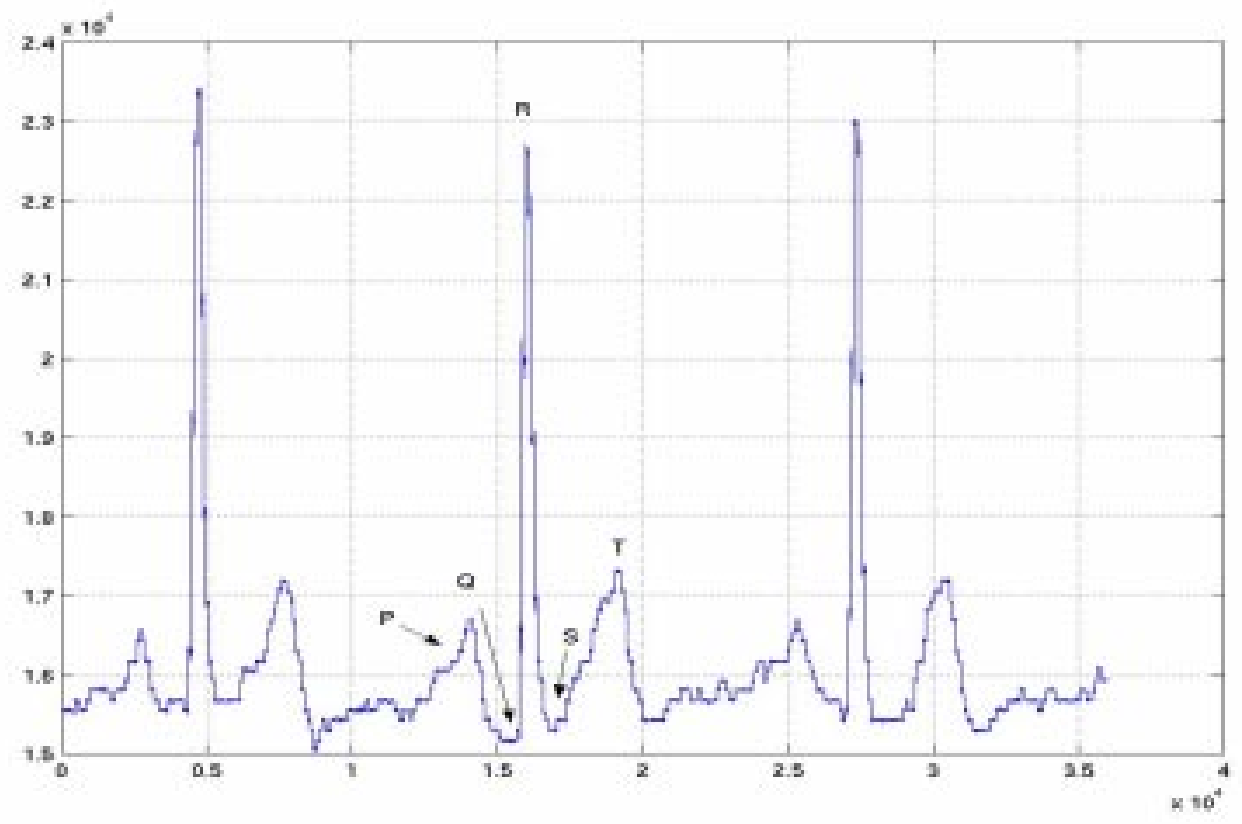

Figure 4.3: Typical ECG signal sampled as $12 \mathrm{kHz}$, where $\mathrm{x}$-axis is sample points and yaxis is relative amplitude. 


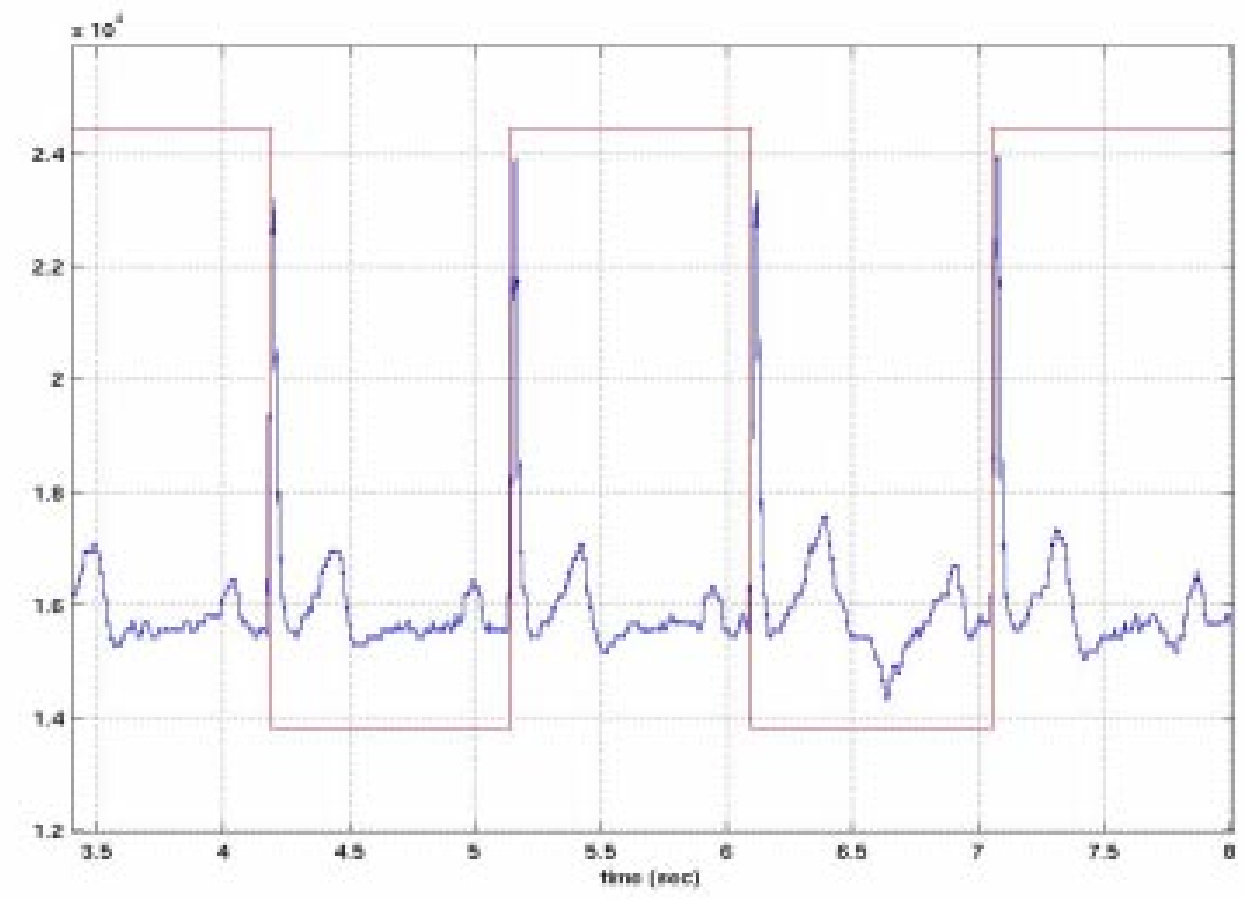

Figure 4.4: Output of trigger program overlaid on the electrocardiogram where the vertical lines are the best locations.

The hybrid algorithm is performed on a beat-to-beat basis using the trigger set. Before the beat-to-beat analysis, some preprocessing of Doppler shift signal is done to estimate the background noise. It is known that the flow of blood in aorta is not present for the entire duration of a cardiac cycle. The power spectrogram of the signal when there is no blood flow can be assumed due to the presence of noise only. The useful signal can be considered to be absent when there is no blood flow during the cardiac cycle. It has been asserted that blood flow is present during a maximum of $66 \%$ of time during a cardiac cycle, which is considered signal plus noise. [Her88] In the power distribution function of the Doppler shift signal, the lower 33\% can be considered noise. 


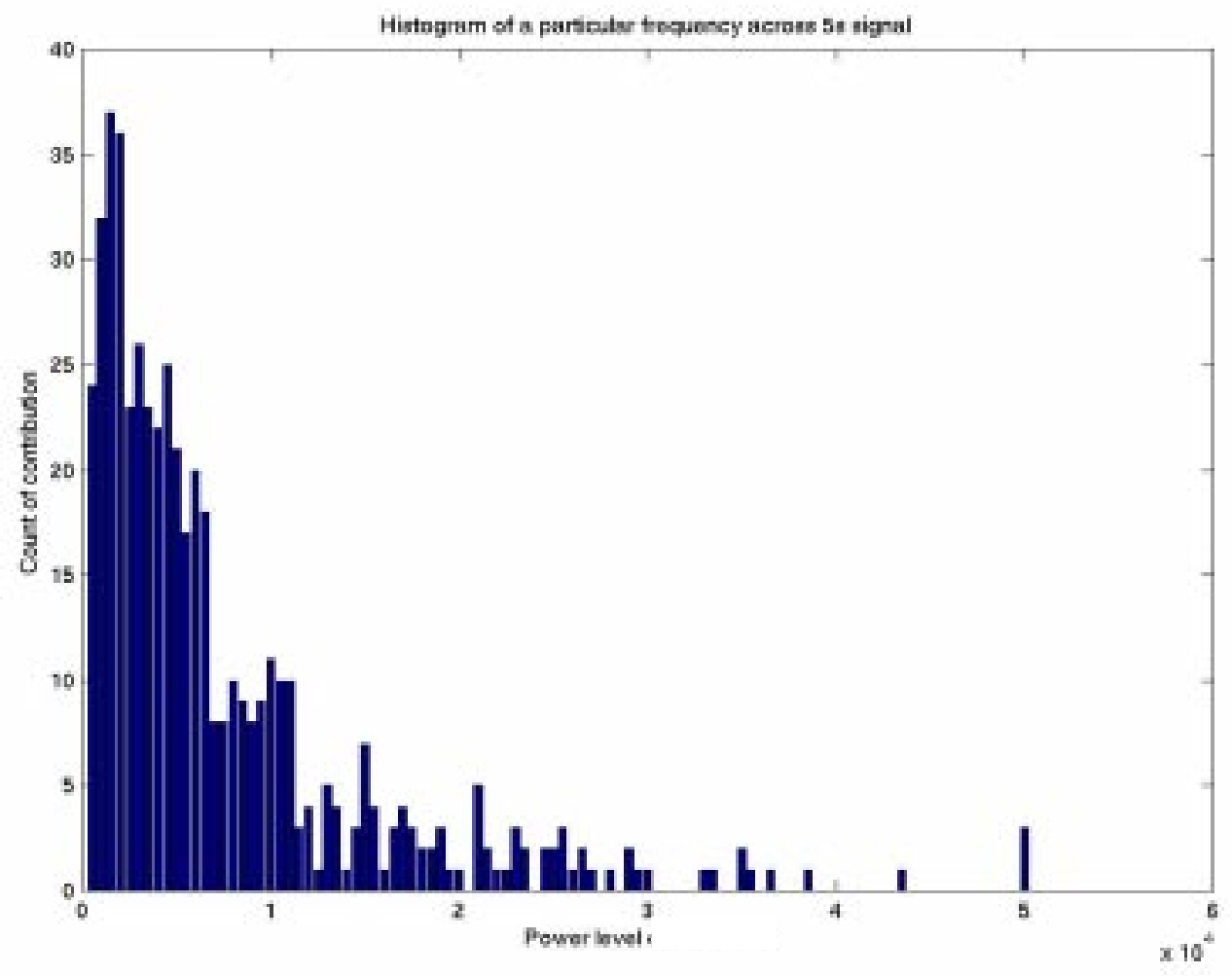

Figure 4.5: Histogram used to determine noise vector, the $\mathrm{x}$-axis is power level and $\mathrm{y}$ axis is frequency or "count". One histogram is created over 5 seconds at a single frequency.

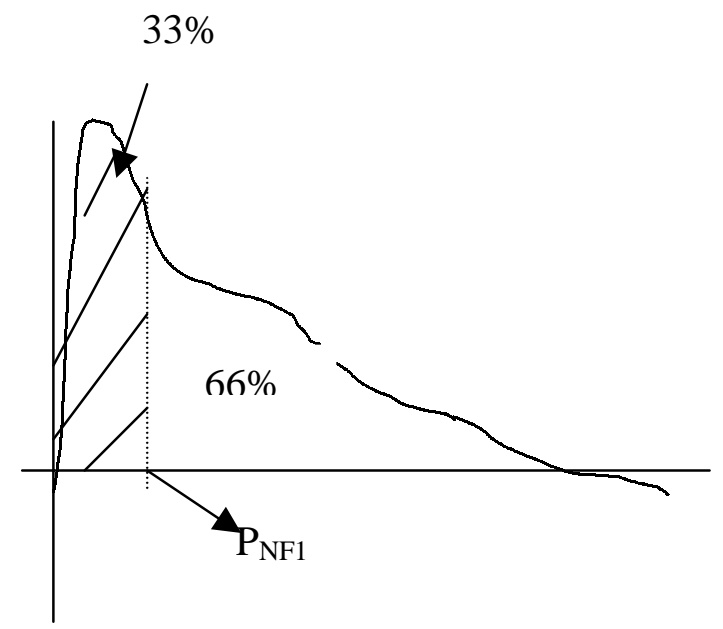

Figure 4.6: Histogram for frequency F1 
The preprocessing function can take this into account such that the noise can be directly subtracted from the shift signal in order to get zero mean background noise. In order to do this, the algorithm divides the entire signal sequence into 5-second signal portions. 120-point blocks (or $10 \mathrm{~ms}$ ) of signal are passed through a 120-point Hanning window and Fourier transform. Histograms are created across each frequency component as shown in figure 4.5 and figure 4.6. The lower $33 \%$ of the area under the histogram is considered noise and the upper $66 \%$ signal. The power level at $33 \%$ is used as the underlying noise contribution at that frequency. This is repeated for all 120 frequencies. Those "average" noise levels are subtracted from the Fourier transform of each individual spectra. [Figure 4.7]

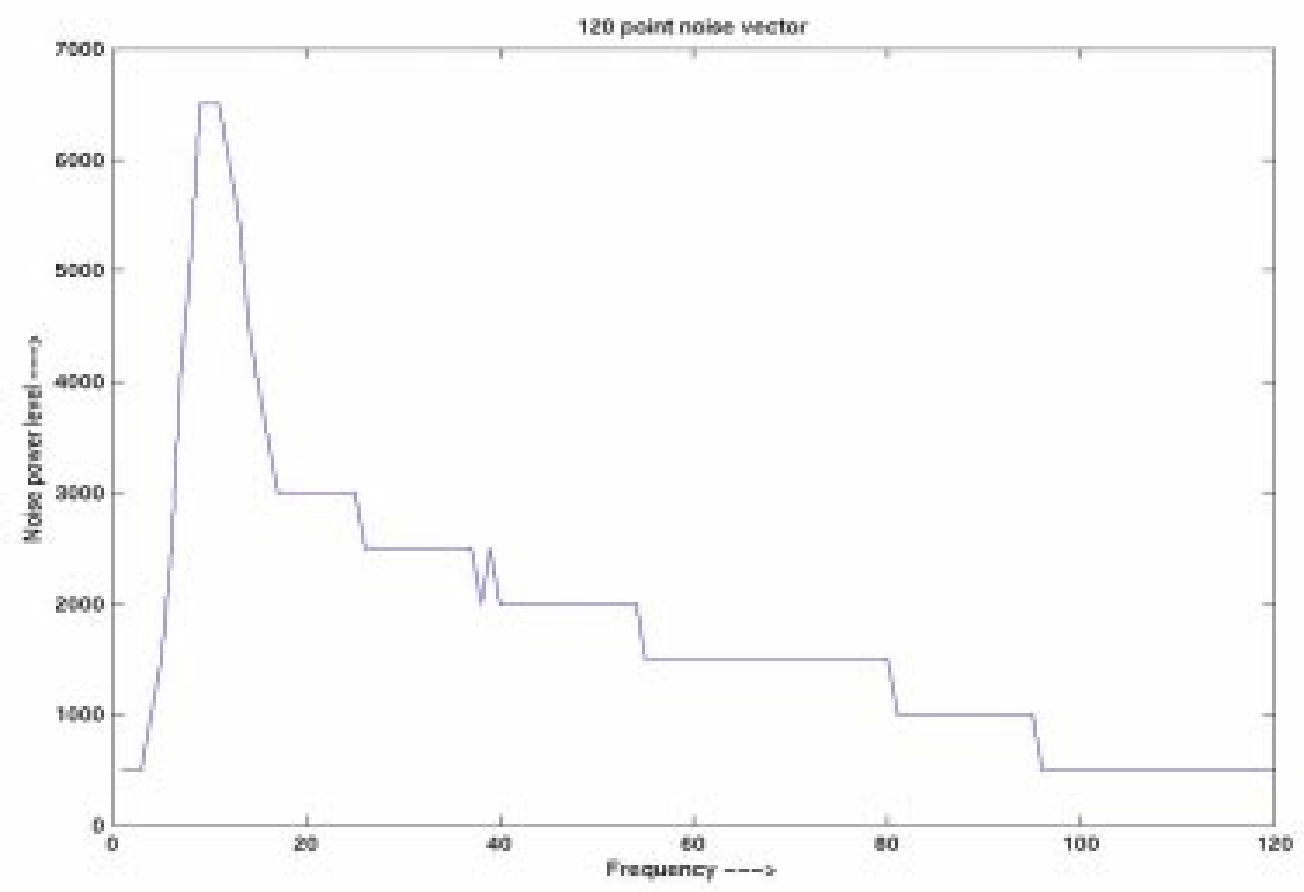

Figure 4.7: A typical calculated noise vector, which is calculated from a histogram for each frequency where noise power level is isolated. This vector is subtracted from each raw Fourier transform of 10-ms window. 
In the beat-to-beat analysis the absolute value of the fast Fourier transforms of the Hanning windowed, 10 millisecond, Doppler shift signal is taken. The average noise vector calculated from the preprocessor stage is subtracted from it and any negative powers that arise due to this are called zeros. The next step is to select the peak frequency of the spectra based on the percentile method. The principle involved in the percentile method [Mo88] is that a threshold, which is a percentage of the total signal plus noise power of a spectrum, can estimate the peak frequency. If $\varphi_{\mathrm{T}}$ is the total power of the spectrum then the peak frequency can be defined as

$$
\text { Fpeak }=(100-\alpha) \varphi_{T} \div 100
$$

Where $\alpha$ is a parameter that depends upon the SNR. The typical value of $\alpha$ is $2 \%$ to $10 \%$ for a high SNR as seen in the Doppler shift spectrum. This gives a reliable estimate of the peak frequency. The basic flow chart of the algorithm is shown in the Appendix 2.

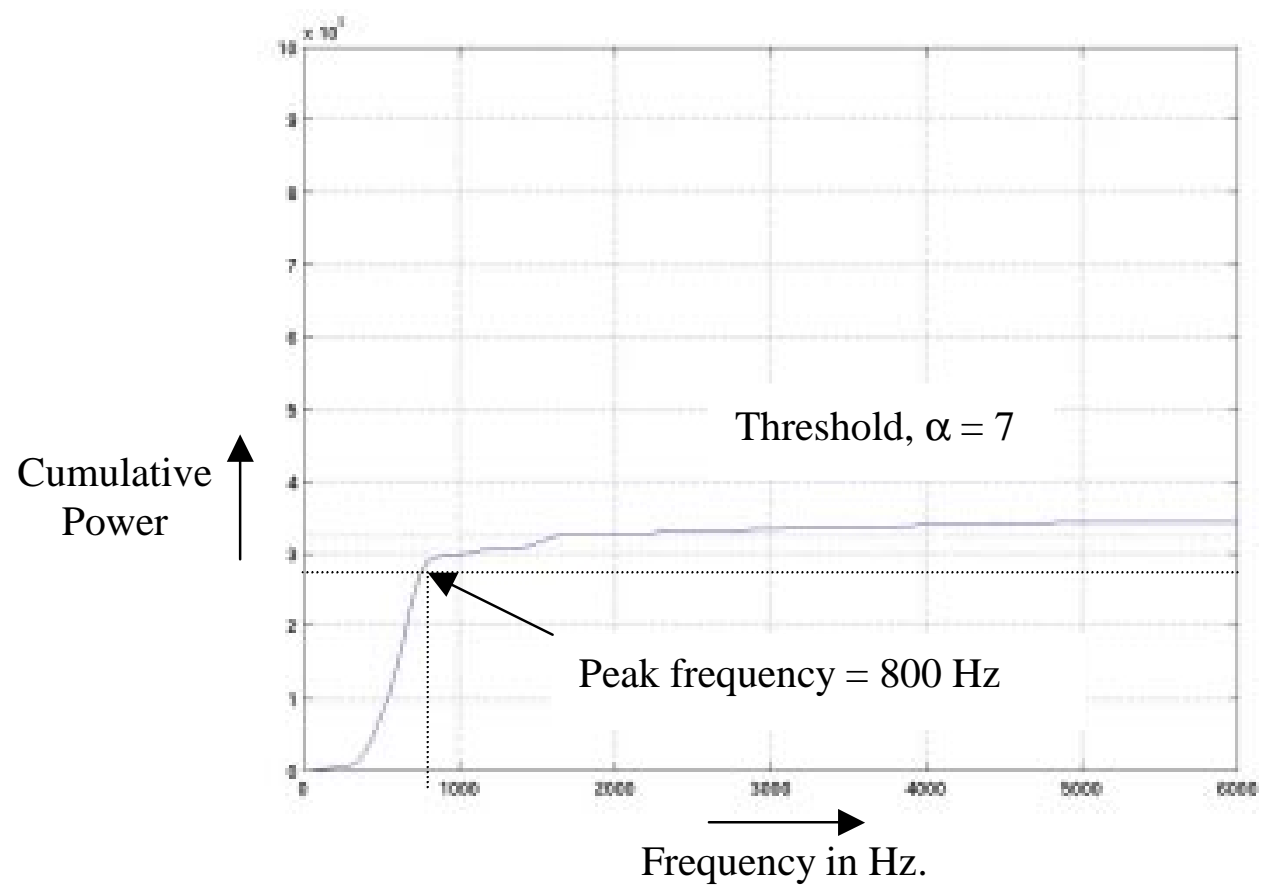

Figure 4.8: Cumulative sum of power (y-axis) for a typical block of signal when there is blood flow. Peak frequency is defined as the change in cumulative power from signal/ noise to only noise. This corresponds to the "knee" in the cumulative power graph. 
To implement this method, the cumulative sum of the powers across the 120 points of the signal block is taken as shown in figure 4.8 and figure 4.9. A percentage of the total sum of power is taken as a threshold value. The peak frequency is the frequency where the threshold coincides with the cumulative sum curve. Note that when signal is present the cumulative power curve has a high slope ( 0 to $800 \mathrm{~Hz}$ on figure 4.8) and when only noise is present, the slope is quite low (Figure 4.9 and over $800 \mathrm{~Hz}$ on figure 4.8).

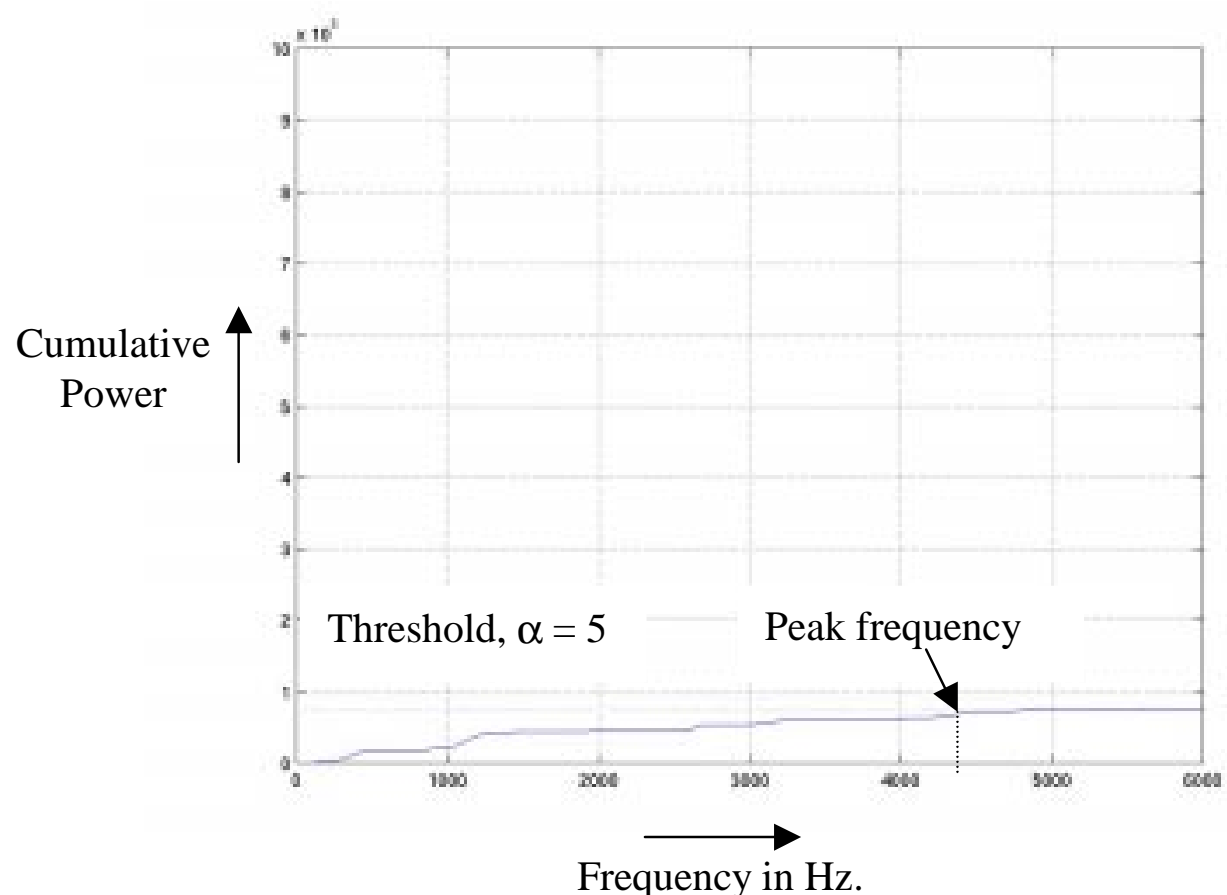

Figure 4.9: Cumulative sum of power (y-axis) for a typical block of signal when there is no blood flow. Peak frequency should not be calculated on this. 
The peak frequency is converted to peak velocity. An example of peak frequency and peak velocity over one beat is given in figure 4.10. The peak velocity is smoothened using a standard smoothing function where a weighted average of neighboring points is taken, neutralizing variability in the peak velocity envelope. This is done by taking five point sliding window and summing the half of the central point, three sixteenth of the adjacent points and one sixteenth of the far points. The peak velocity is plotted against the time. The area under this curve gives the stroke volume integral. This multiplied by the cross sectional area of aorta gives the stroke volume.
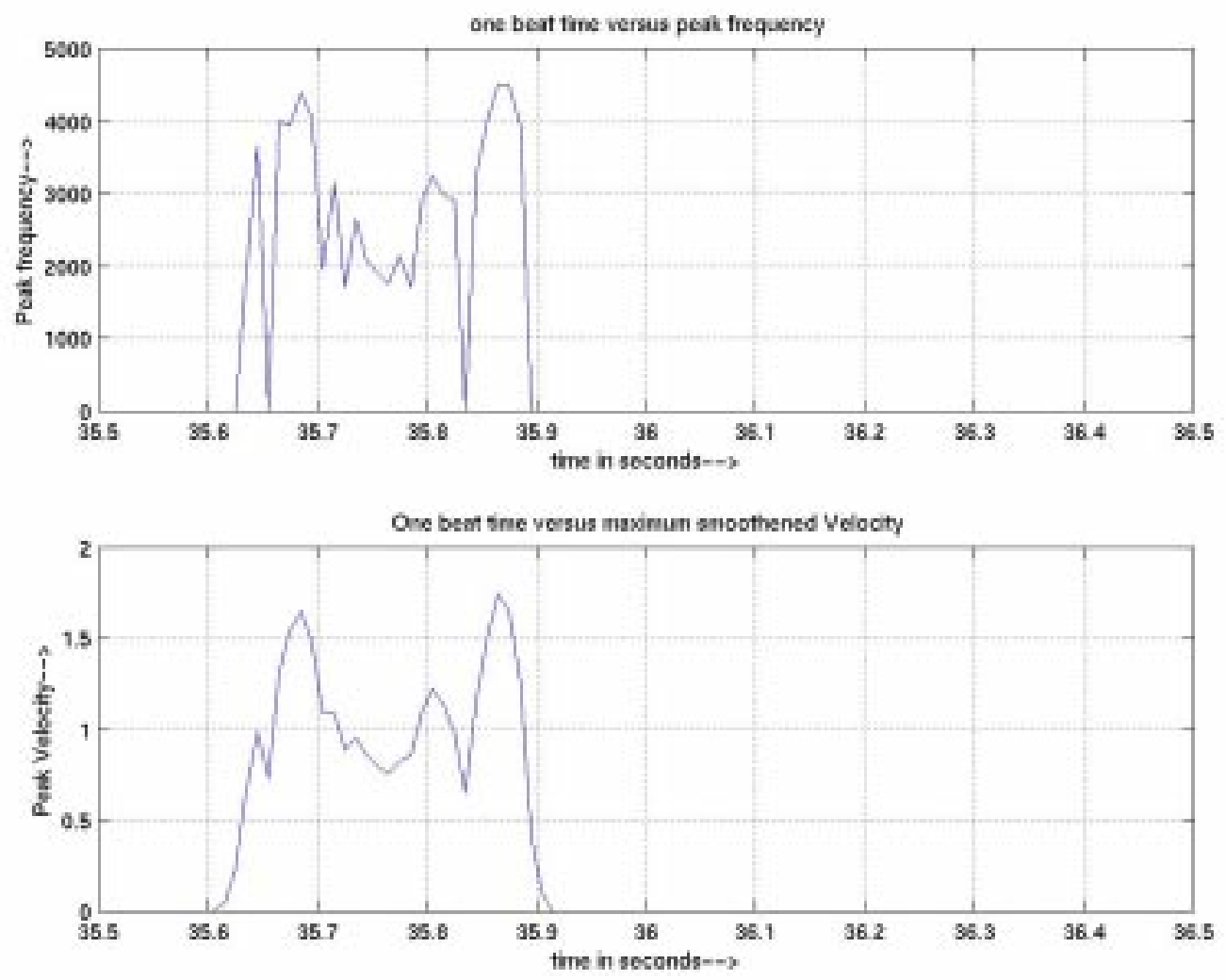

Figure 4.10: Peak frequency versus time (top) and smoothened velocity (bottom) where peak frequency or velocity is calculated every $10 \mathrm{~ms}$. 
The trigger set gives the number of samples between two cardiac cycles. This information is used to find out the instantaneous heart rate. The product of stroke volume and the instantaneous heart rate gives the instantaneous cardiac output. The output of the algorithm is a result file, which gives the beat number, instantaneous heart rate, stroke volume integral and stroke volume.
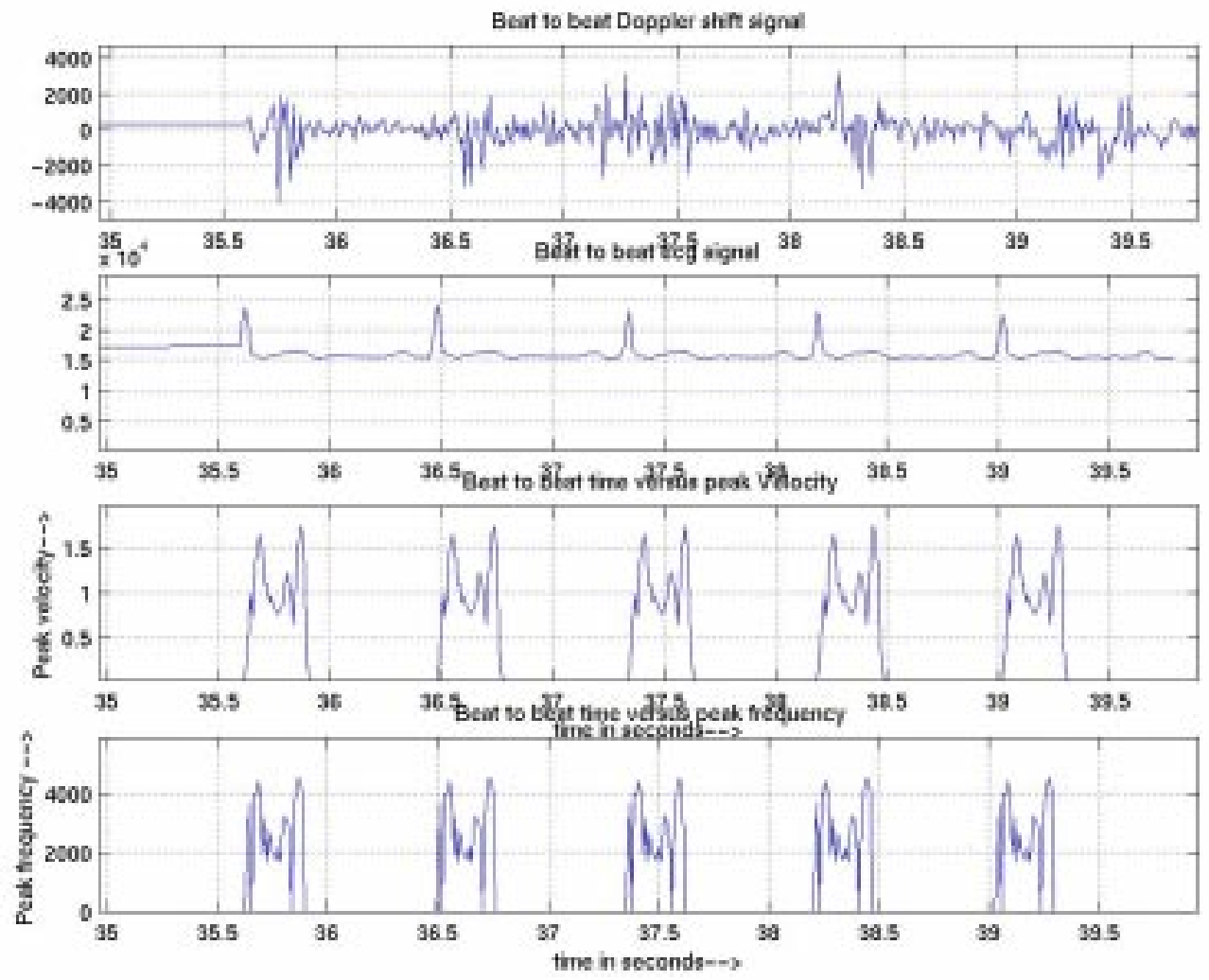

Figure 4.11: Beat to beat view of the output of the algorithm where the top two graphs are the Doppler shift and the ECG respectively and the bottom two graphs are smoothened peak velocity and peak frequency respectively. 
The hybrid percentile algorithm is an attempt to integrate the findings of various papers [Eri90] [Her88] [Mo88] and [Ric96] and takes into account the behavior of the Doppler shift signal on application of various constraint. The first achievement is the discrimination of the signal when there is blood flow and when there is no blood flow. This is done using the cumulative sum of the fast Fourier transforms.

When the percentile threshold was used to pick up the peak frequency from the cumulative sum of the frequencies then those portions of the Doppler shift signal with no blood flow also contributed towards the peak frequency. This gave rise to high stroke volume integral when the peak velocity versus time was plotted. Figure 4.13 shows the general trend of the cumulative sum of the FFT over a complete cardiac cycle. It can be seen in figure 4.12 that there is a huge difference in the power levels of the FFT when blood flow is present and absent. It is observed that there is steep rise with a distinct knee in case of the Doppler shift signal from the flow region. This property can be utilized properly to choose the FFT, which are in the flow region.

After a careful observation of the pattern of the power levels in the flow and nonflow region, a method to determine signal (blood flow present) from noise (blood flow absent) was developed with the use of the cumulative sum of power. This is one of the major contributions of this research. A sudden rise in the cumulative sum of power curve was found throughout the signal when there was blood flow. In comparison, the cumulative sum of power curve for a noisy section of signal when there was no blood flow did not have a steep slope. After observing many of these curves, for portions of the Doppler shift signal that contained blood flow information, it was hypothesized that $60 \%$ (50\% was also tested) of the power of the FFT is reached by $1000 \mathrm{~Hz}$ or $90 \%$ is reached by $2000 \mathrm{~Hz}$. (80\% was also tested). 

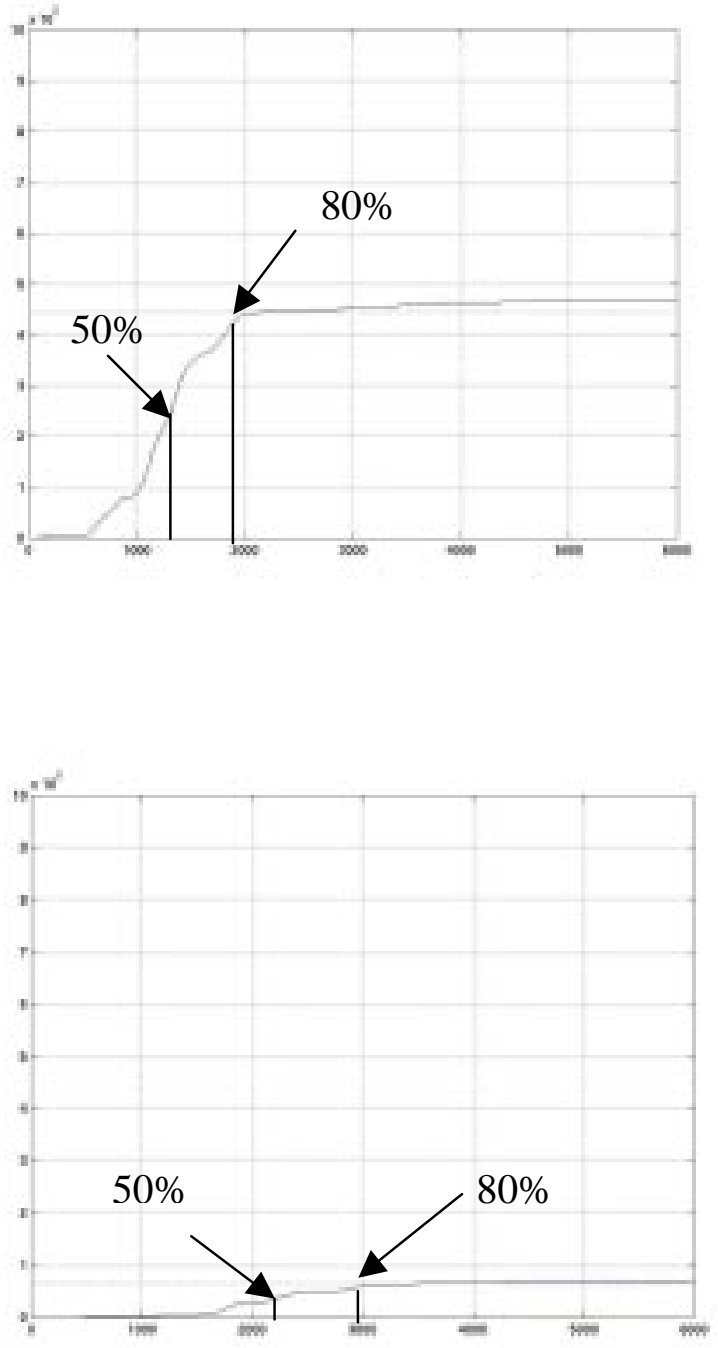

Figure 4.12: Cumulative sum of powers of an FFT of a signal when there is blood flow (above) and when there is no blood flow (below). The basic difference in the slopes of the curve is used for discriminating between a useful section of signal and noise. 


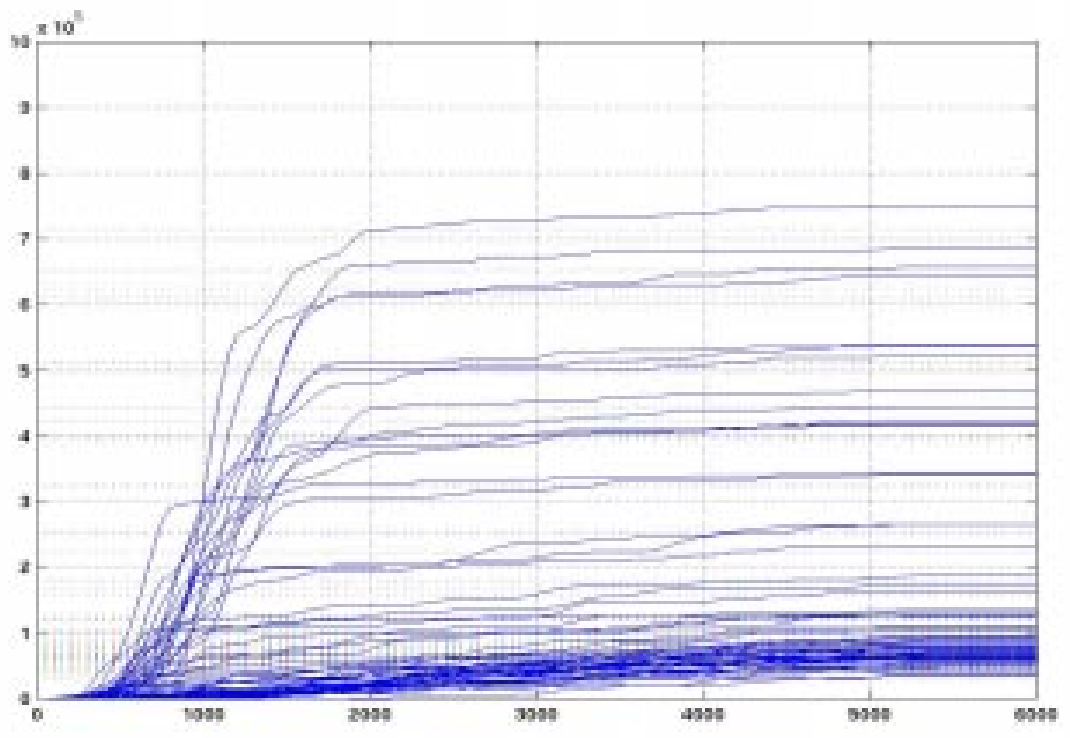

Figure 4.13: Cumulative sum of amplitudes over a complete cardiac cycle. 


\section{CHAPTER 5: Results}




\subsection{RESULT}

Figures 5.1 through 5.6 give the results for the algorithm for different values of the cutoff power level and the threshold (alpha) value. The lower cutoff was tested at $50 \%$ and $60 \%$ and the higher cutoff was $80 \%$ and $90 \%$. The threshold values were selected at 90,93 and 95 percentile. 

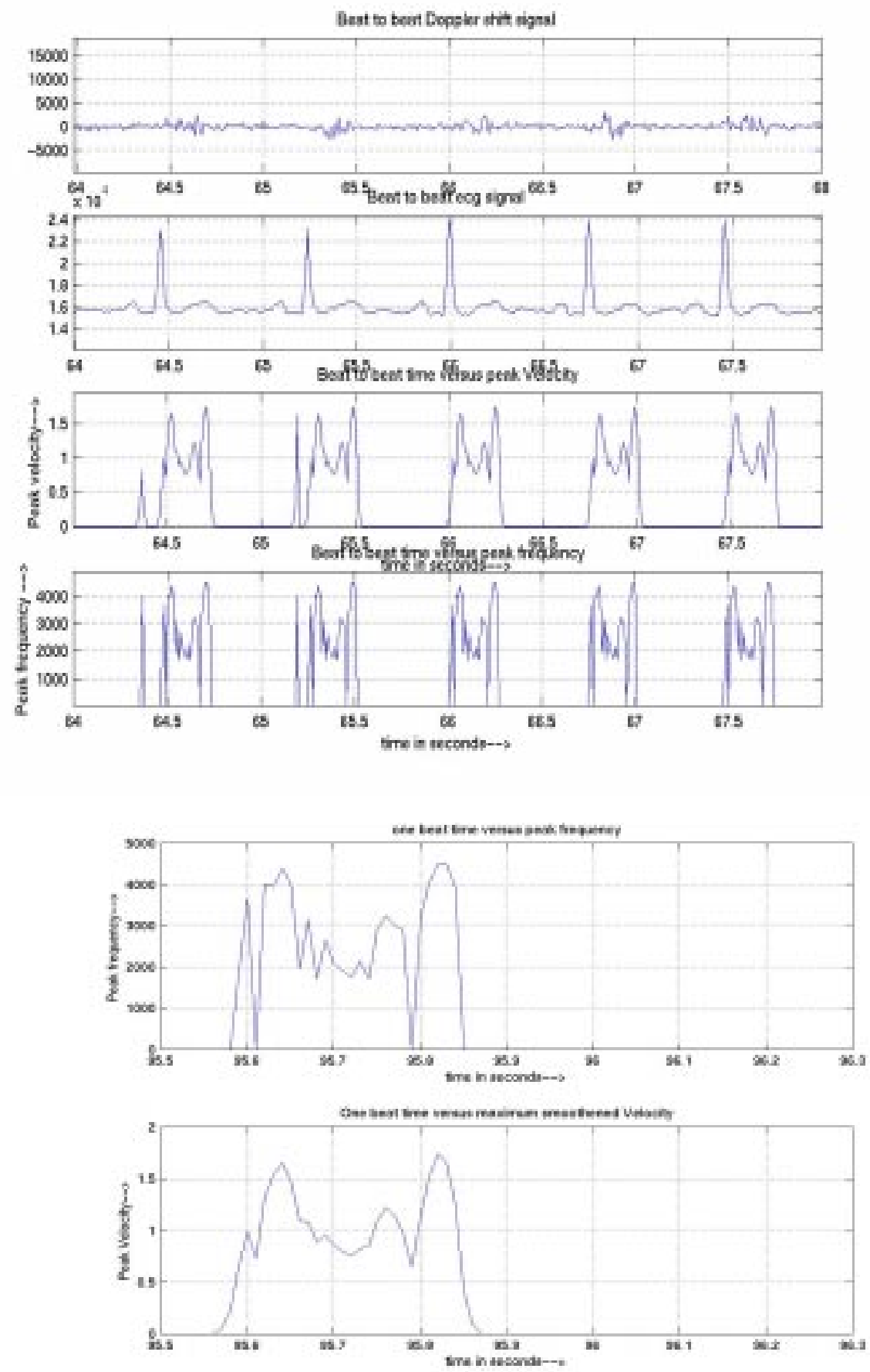

Figure 5.1: Lower cutoff $=50 \%$ power @ $1000 \mathrm{~Hz}$., Higher cutoff $=80 \%$ power @ 2000 Hz., Threshold $=95$ percentile. The top graph is results over five beats with Doppler shift signal, electrocardiogram, smoothened peak velocity and peak frequency, from top to bottom respectively. The bottom graph is peak frequency and smoothened peak velocity for one beat. 

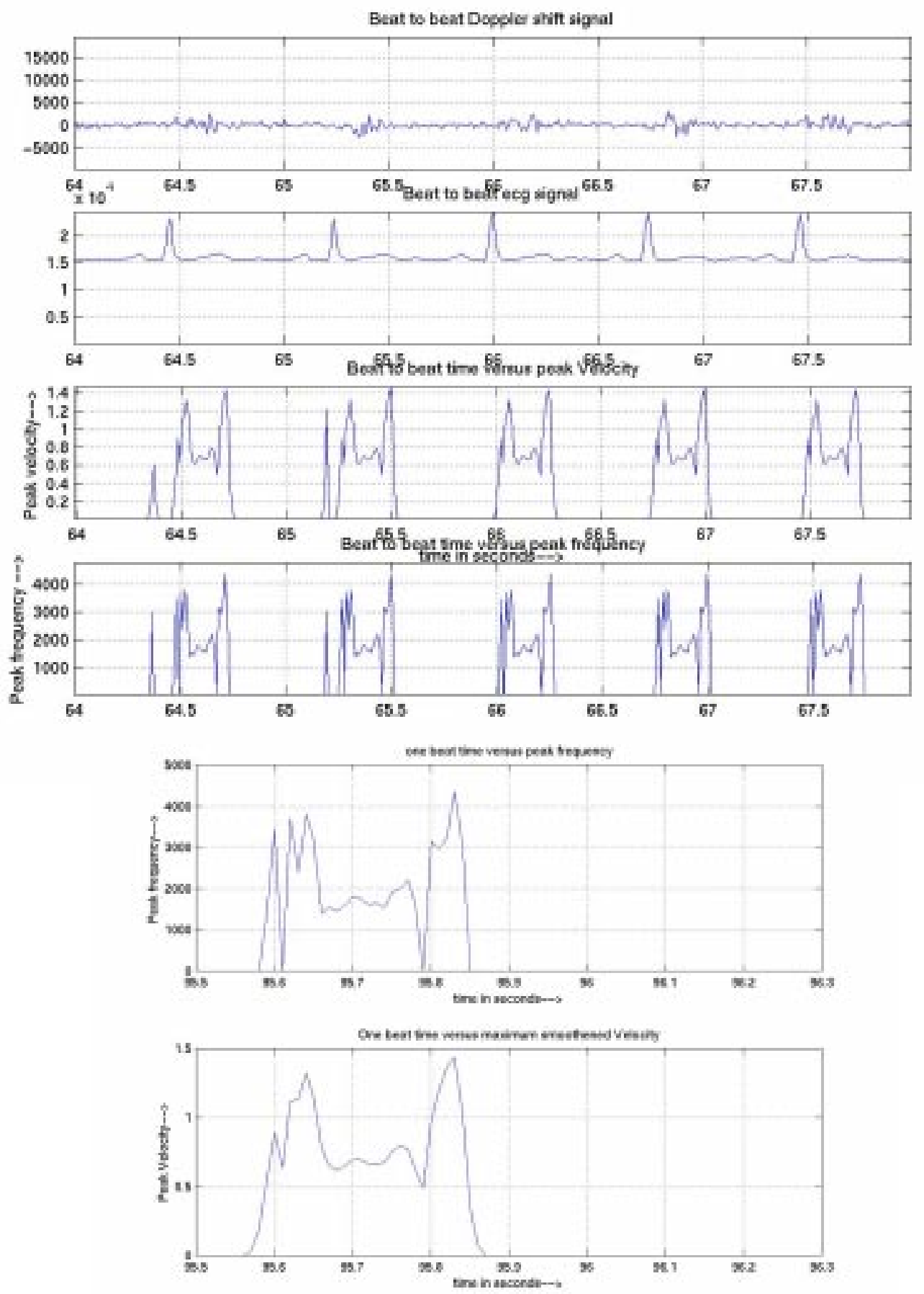

Figure 5.2: Lower cutoff = 50\% power @ 1000 Hz., Higher cutoff =80\% power @ 2000 Hz., Threshold $=90$ percentile. The top graph is results over five beats with Doppler shift signal, electrocardiogram, smoothened peak velocity and peak frequency, from top to bottom respectively. The bottom graph is peak frequency and smoothened peak velocity for one beat. 

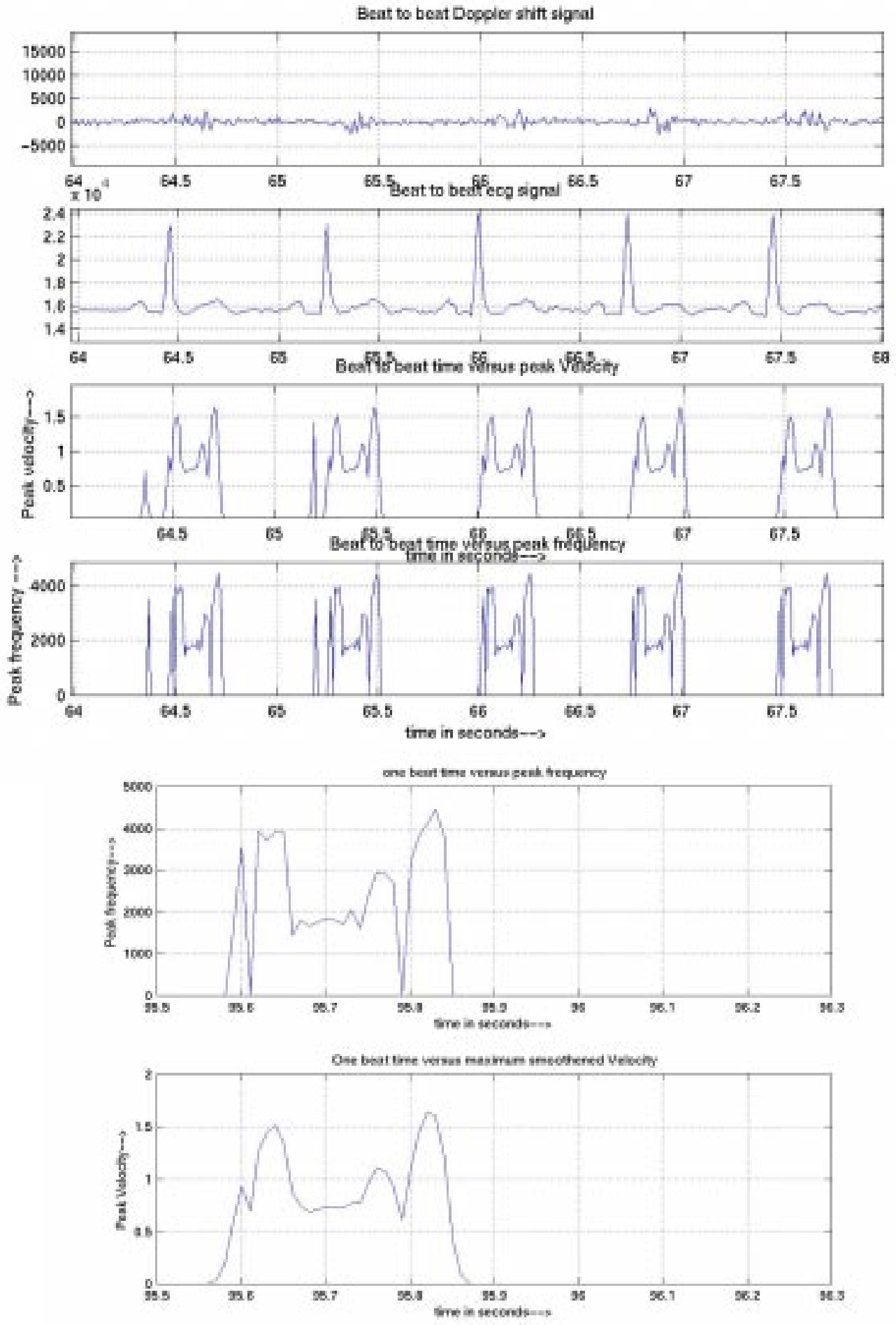

Figure 5.3: Lower cutoff $=50 \%$ power $@ 1000$ Hz., Higher cutoff $=80 \%$ power $@ 2000$ Hz., Threshold $=93$ percentile. The top graph is results over five beats with Doppler shift signal, electrocardiogram, smoothened peak velocity and peak frequency, from top to bottom respectively. The bottom graph is peak frequency and smoothened peak velocity for one beat. 

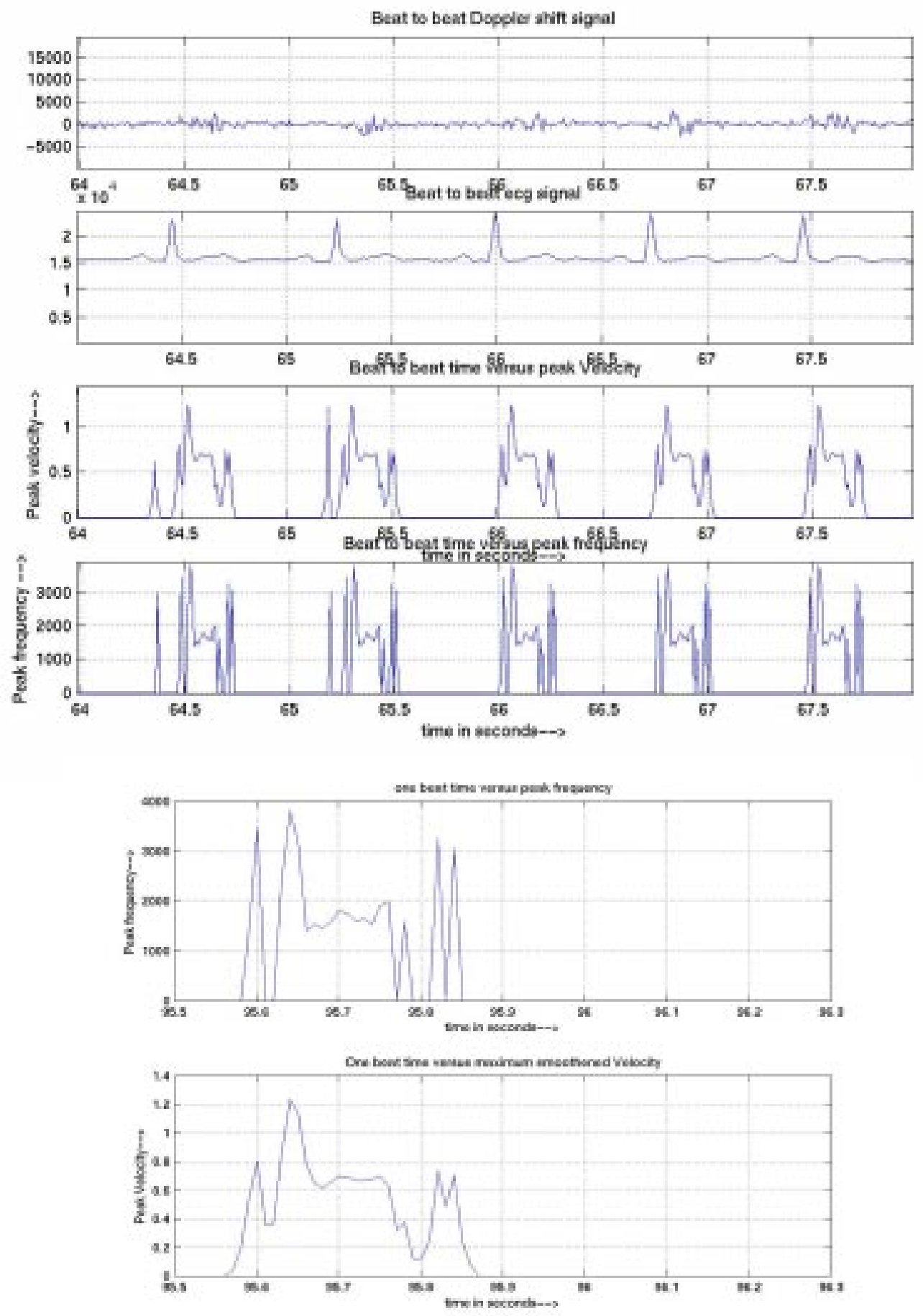

Figure 5.4: Lower cutoff $=60 \%$ power $@ 1000$ Hz., Higher cutoff $=90 \%$ power @ 2000 Hz., Threshold $=90$ percentile. The top graph is results over five beats with Doppler shift signal, electrocardiogram, smoothened peak velocity and peak frequency, from top to bottom respectively. The bottom graph is peak frequency and peak velocity for one beat. 

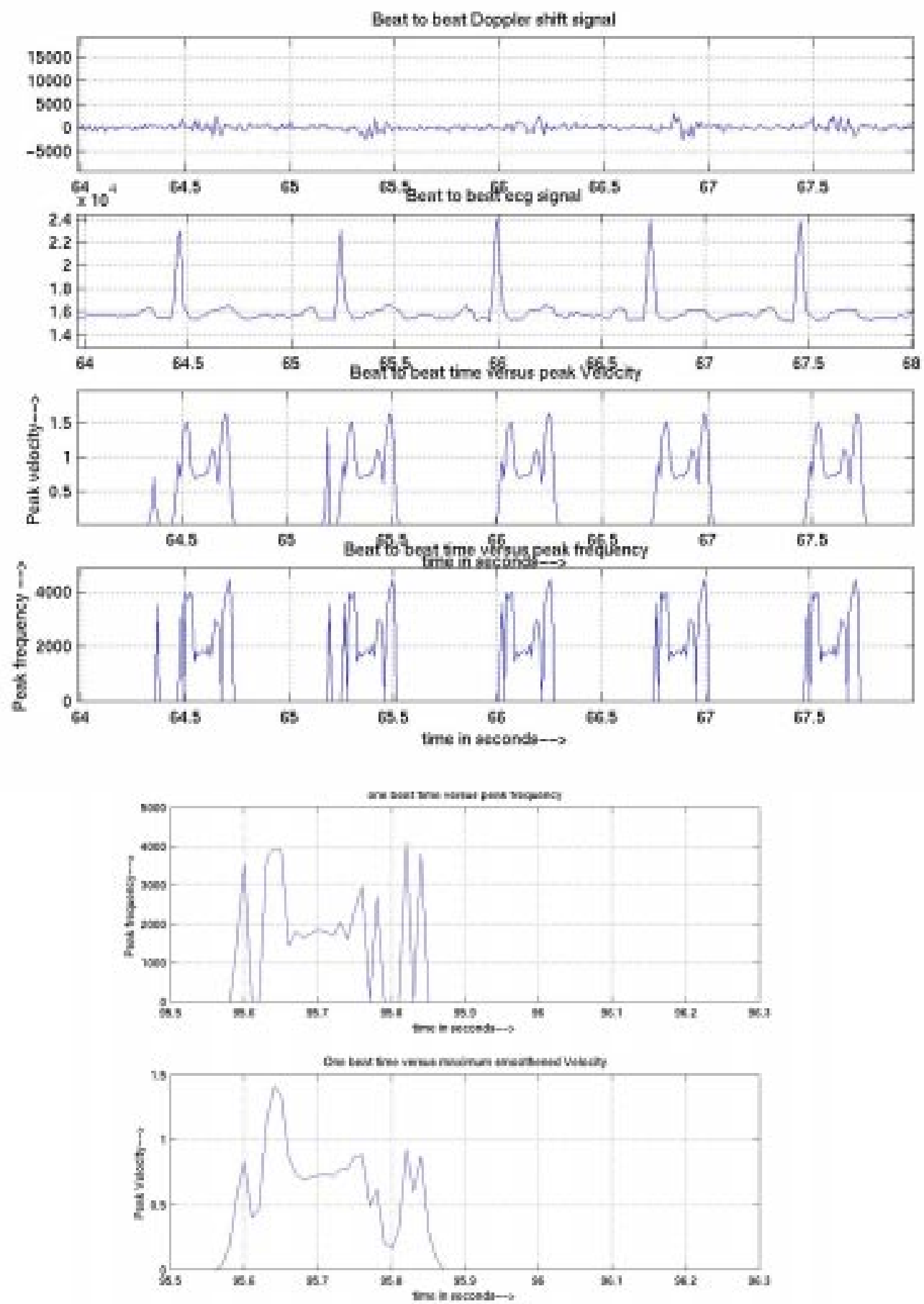

Figure 5.5: Lower cutoff $=60 \%$ power @ $1000 \mathrm{~Hz}$, Higher cutoff =90\% power @ 2000 Hz., Threshold $=93$ percentile. The top graph is results over five beats with Doppler shift signal, electrocardiogram, smoothened peak velocity and peak frequency, from top to bottom respectively. The bottom graph is peak frequency and peak velocity for one beat. 

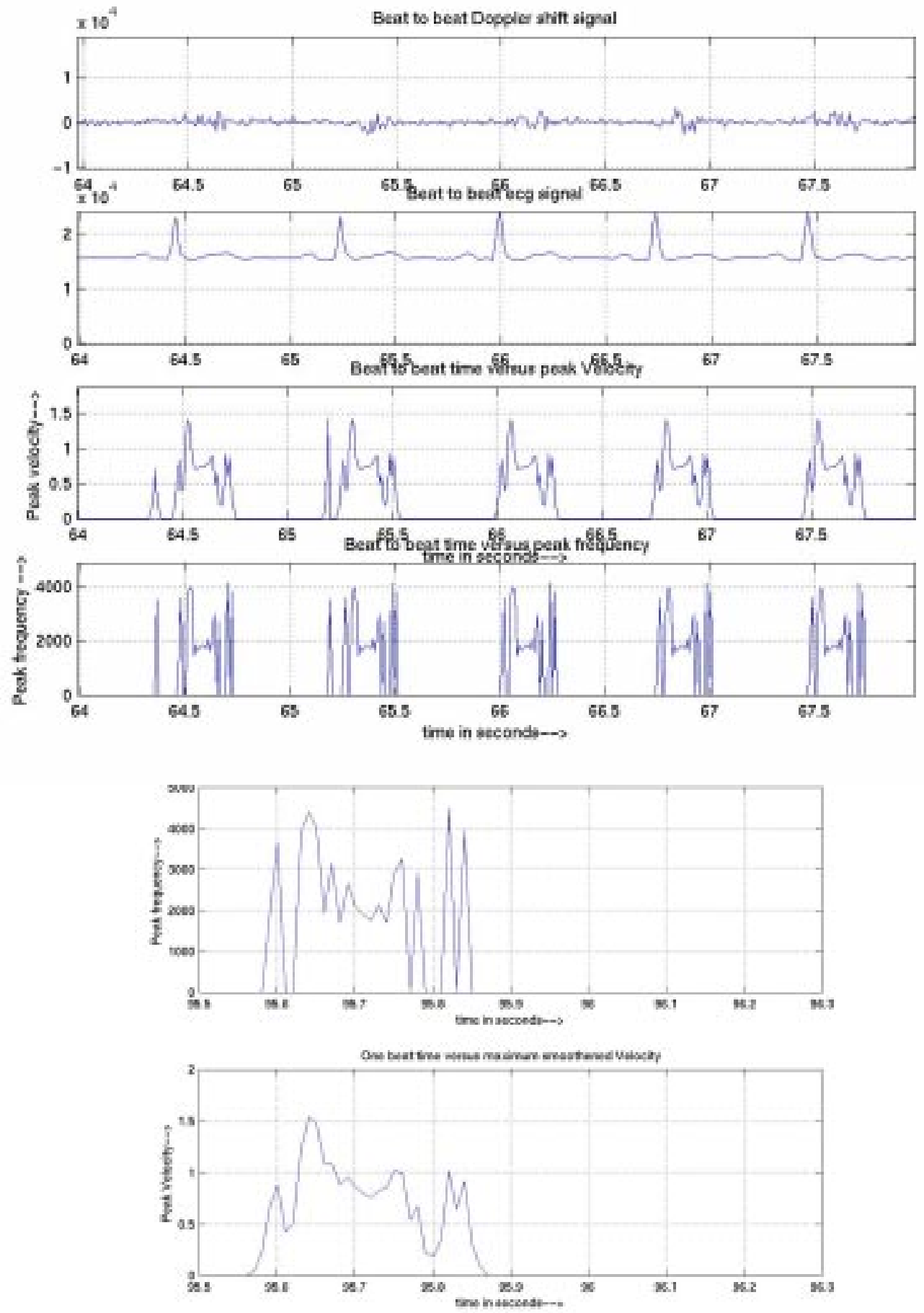

Figure 5.6: Lower cutoff $=60 \%$ power $@ 1000$ Hz., Higher cutoff $=90 \%$ power @ 2000 Hz., Threshold $=95$ percentile. The top graph is results over five beats with Doppler shift signal, electrocardiogram, smoothened peak velocity and peak frequency, from top to bottom respectively. The bottom graph is peak frequency and smoothened peak velocity for one beat. 
Reference values of stroke volume were calculated manually (see chapter 4). Heart rate was used to align the reference values with the raw data used by the algorithm in this thesis. (Figure 5.7) Figure 5.8 is a comparison of the calculated stroke volume (SV) for each of the parameter sets.

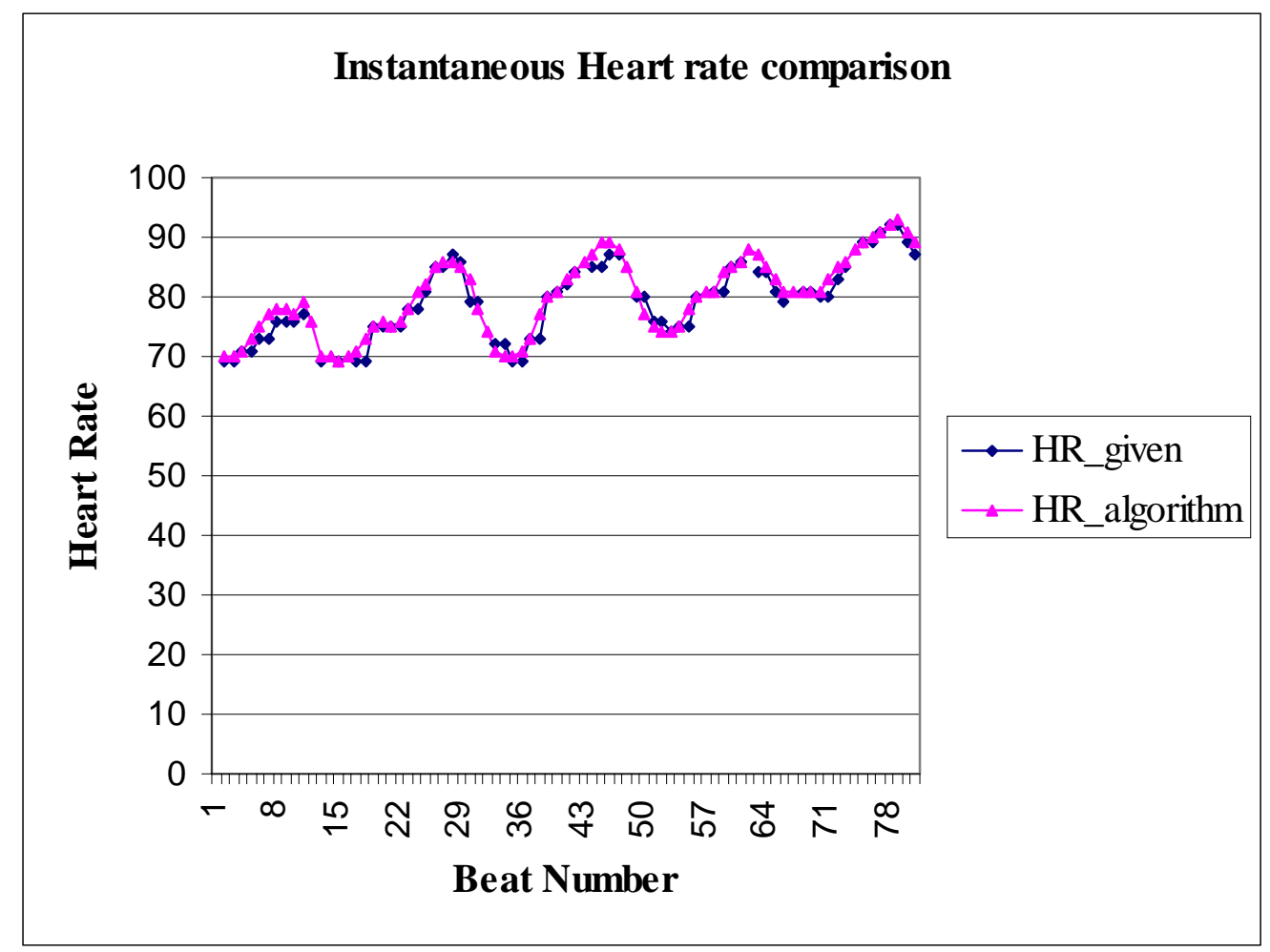

Figure 5.7: Heart rate comparison for referenced data versus calculated heart rate from this algorithm. 


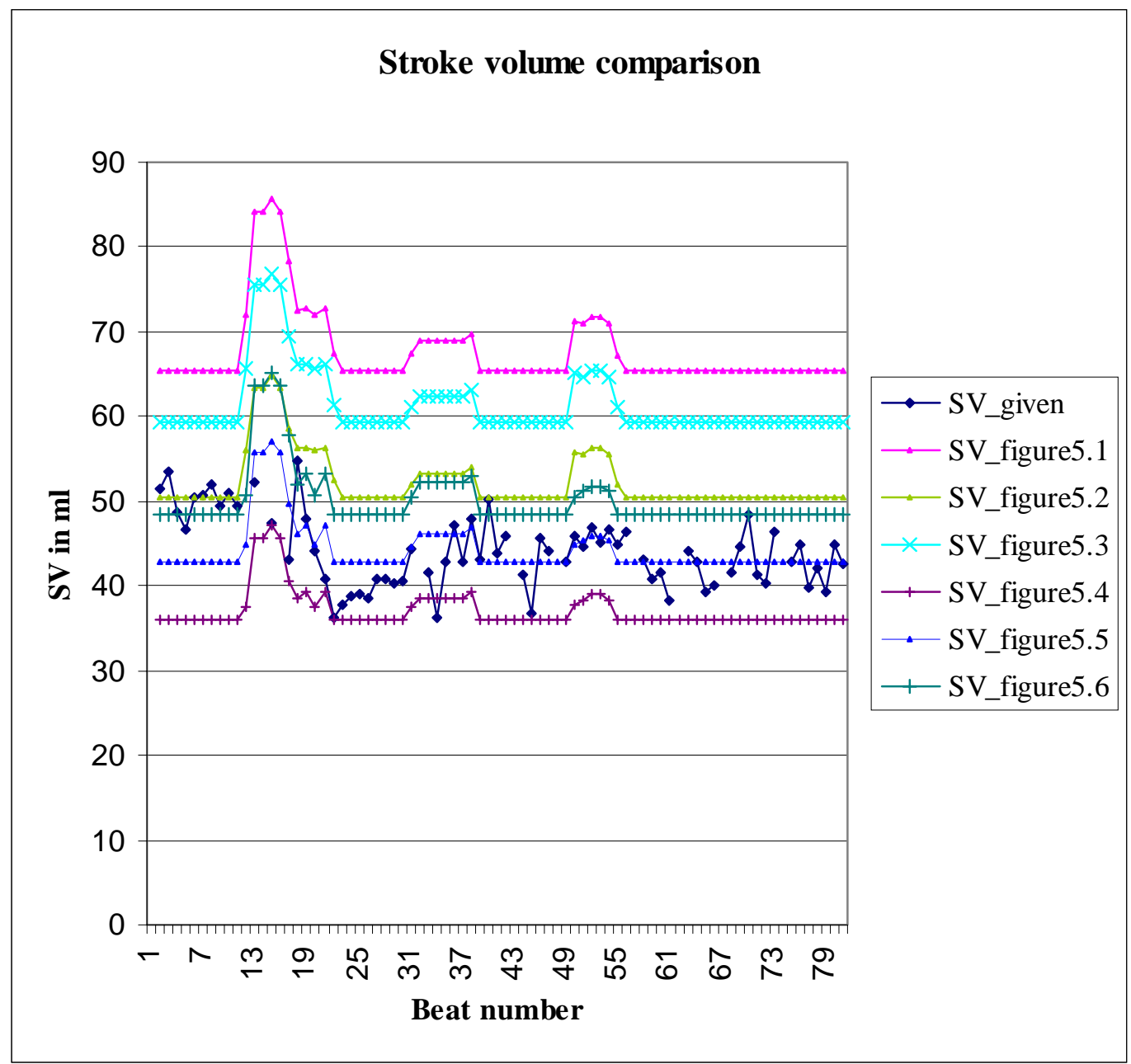

Figure 5.8: Comparison of stroke volume for the different parameter sets given in figures 5.1 through 5.6 . 
In the comparison of calculated the stroke volume to the reference, the settings corresponding to the figure 5.5 (Lower cutoff $=60 \%$ power $@ 1000 \mathrm{~Hz}$., Higher cutoff = 90\% power @ $2000 \mathrm{~Hz}$. , Threshold = 93 percentile) have the best correlation $(\rho=.34)$ with the reference data as shown in figure 5.9.

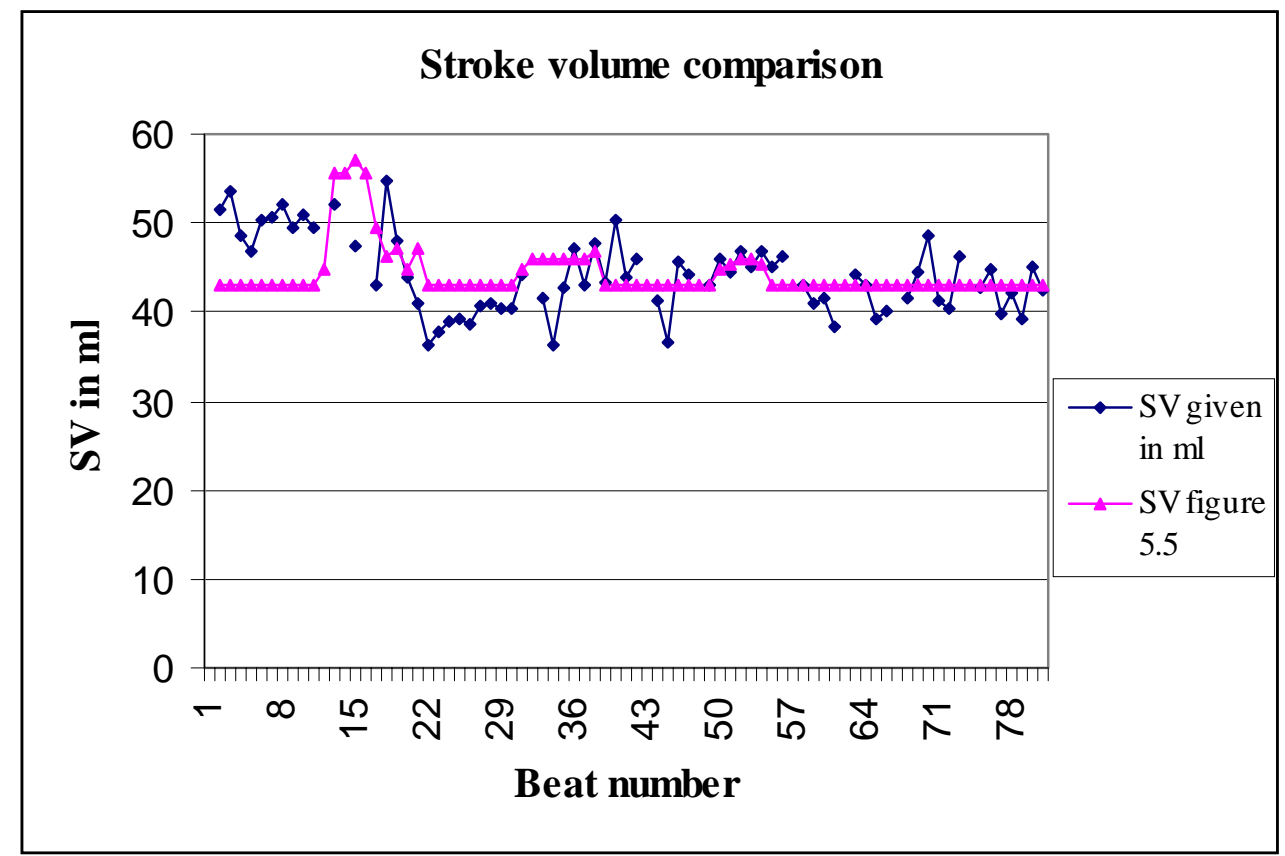

Figure 5.9: Stroke volume comparison for best setting (Lower cutoff $=60 \%$ power @ 1000 Hz., Higher cutoff = 90\% power @ 2000 Hz., Threshold = 93 percentile) versus the reference values over multiple beats. 


\section{CHAPTER 6: Conclusion and Future work}


This thesis developed a new method for detecting the peak frequency versus time such that stroke volume can be calculated. This method includes a combination of techniques: subtraction of a noise vector from the FFT of the Doppler shift signal [Her88]; differentiation of signal and noise through the cumulative sum of powers; and the selection of peak frequency from spectra using percentile method [Mo88]. However, more development and analysis work is needed before this algorithm can be used for research or clinically.

Clearly, in comparison with the reference values, the calculated stroke volume is not highly correlated. There are several hypothesized reasons for these differences. First, it is possible that the last "spike" in the peak velocity curve is due to the contribution of the windkessel effect and the reverse flow of blood in the Doppler shift signal. [Kre95] In the arterial region the blood flow is pulsatile and is directly experiencing the effects of the beating heart. The above two characteristics are dominant. In the cardiac cycle, with the contraction of the ventricle the fluid is forced into the aorta such that the aorta expands and the volume within it increases. Later, when the driving pressure is reduced the aorta contracts producing extended flow. This is known as windkessel effect. This results in the continued flow of the fluid in the forward direction. In the distal circulation, due to the decrease in pressure and contraction of the vessel, there is reversal of flow. Hence the forward and reverse flow is observed within the arterial circulation. This may potentially add to the Doppler shift signal after the systolic activity. Future work will be to "filter" out this information before calculation of stroke volume.

Second, the method of location of the knee in the cumulative sum of the FFT curve should be improved. Often the percentage of total power method does not choose the optimal point of the knee but rather above the knee in the noise (making the peak frequency estimate too high) or below (making the peak frequency estimate too low). The first derivative of the cumulative sum of curve can be taken and the first minima after the first maxima can be considered as the point of peak frequency. 
Third, it is not clear whether the "reference" values are truly the optimal values for the stroke volume. These values were taken from manual tracings on the screen, which clearly have much variability, real or otherwise. Future work will be to test this algorithm on more reliable data gathered from NASA or from simulations. In addition, once the algorithm is further developed, it will be necessary to evaluate it on a larger data set. The data should be recorded from different individuals under different clinical conditions. 


\section{References}




\section{REFERENCES}

[Ang85] ANGELSEN BJORN and HATLE LIV, Doppler Ultrasound in Cardiology: Physical Principles and Clinical Applications, second edition, Lea \& Febiger, 1985

[Bak70] BAKER DONALD W, Pulsed Ultrasonic Doppler Blood-Flow Sensing, IEEE transactions on Sonics and ultrasound, vol., su-17, no. 3, July 1970

[Ber90] BERNE ROBERT M., LEVY MATHEW N., The Principles of Physiology; C.V. Mosby Company, 1990.

[Bow91] BOWDLE ANDREW T., FREUND PETER R., and ROOKE ALEC G., Biophysical Measurement series: Cardiac Output; Space Labs Inc., 1991

[Cob82] COBBOLD R.S.C., KASSAM M. JOHNSTON K.W., Online identifying and quantifying Doppler ultrasound waveforms, Med. \& Biol. Eng. \& Comput., 1982, 20, 336-342

[D'A185] D'ALESSIO T., Objective algorithm for maximum frequency estimation in Doppler spectral analysis, Med \& Biol. Eng. \& Comput., 1985,23,63-68

[Eri90] ERIKSEN M. and WALLOE L., Improved method for cardiac output determination in man using ultrasound Doppler technique, Med \& Biol. Eng. \& Comput., 1990,28,555-560

[Fei94] FEIGENBAUM HARVEY, Echocardiography, Fifth edition, Lea \& Febiger. 1994. 
[Fri96] FRITSCH-YELLE JM, WHITSON PA, BONDAR RL, BROWN TE, Subnormal norepinephrine release relates to presyncope in astronauts after spaceflight; J. Appl Physiology, 81:2134-2141, 1996.

[Go196] GOLDBERG BARRY and PETTERSSON HOLGER, Ultrasonography: The NICER Year Book 1996, ISIS Medical Media, 1996

[Her88] HERINGA A., DANIELS O et al., Computer processing of cardiac Doppler signals, Med \& Biol. Eng. \& Comput., 1988,26,147-152

[Kre95] KREMKAU FREDERICK W, Doppler Ultrasound - Principles and Instruments, Second edition, W.B. Saunders, 1995

[Kre98] KREMKAU FREDERICK W, Diagnostic Ultrasound - Principles and Instruments, Fifth edition, W.B. Saunders, 1998

[Lev95] LEVICK JR, An Introduction to Cardiovascular Physiology, 2nd edition, 1995; Butterworth-Heinemann Ltd.

[Mac90] ROBERT MACDONALD, JANICE JENKINS, et al, A Software trigger for Intracardiac Waveform Detection with Automatic Threshold Adjustment, IEEE, 1990, pp 167-170.

[Mo88] MO LARRY Y.L., LOUIS C.M. YUN and COBBOLD R.S.C., Comparison of four digital Maximum frequency estimators for Doppler Ultrasound, Ultrasound in Medicine and Biology, 1988, Vol. 14, No 5, pp 355-363

[Nan93] NANDA NAVIN C., Doppler Echocardiography, Second edition, 1993, Lea \& Febiger. 
[Ric96] RICKEY D.W. and FENSTER A., Evaluation of an automated real-time spectral analysis technique, Ultrasound in Medicine and Biology, 1996, Vol.22, No 1, pp 61-73

[Rou94] ROUTH H.F., POWRIE C.W. and PETERSON R.B., Continuous display of peak and mean blood flow velocities, US patent 5,287,753, Feb. 22, 1994

[Sai93] SAINI VASANT D., Doppler Signal analyses, Lea \& Febiger, 1993.

[Taylor] TAYLOR KENNETH J.W., BURNS PETER N., WELLS PETER NT, Clinical Application of Doppler Ultrasound, 2nd edition, Raven press.

[Wey94] WEYMAN ARTHUR E., Principles and Practice of Echocardiography, Second edition, Lea \& Febiger, 1994.

[www1] Biosound Esaote Inc. http://www.biosound.com

[www2] TEAC America Inc. http://www.teac.com 
Appendix (1) : Programs 


\section{PROGRAMS}

\section{function maintry(data)}

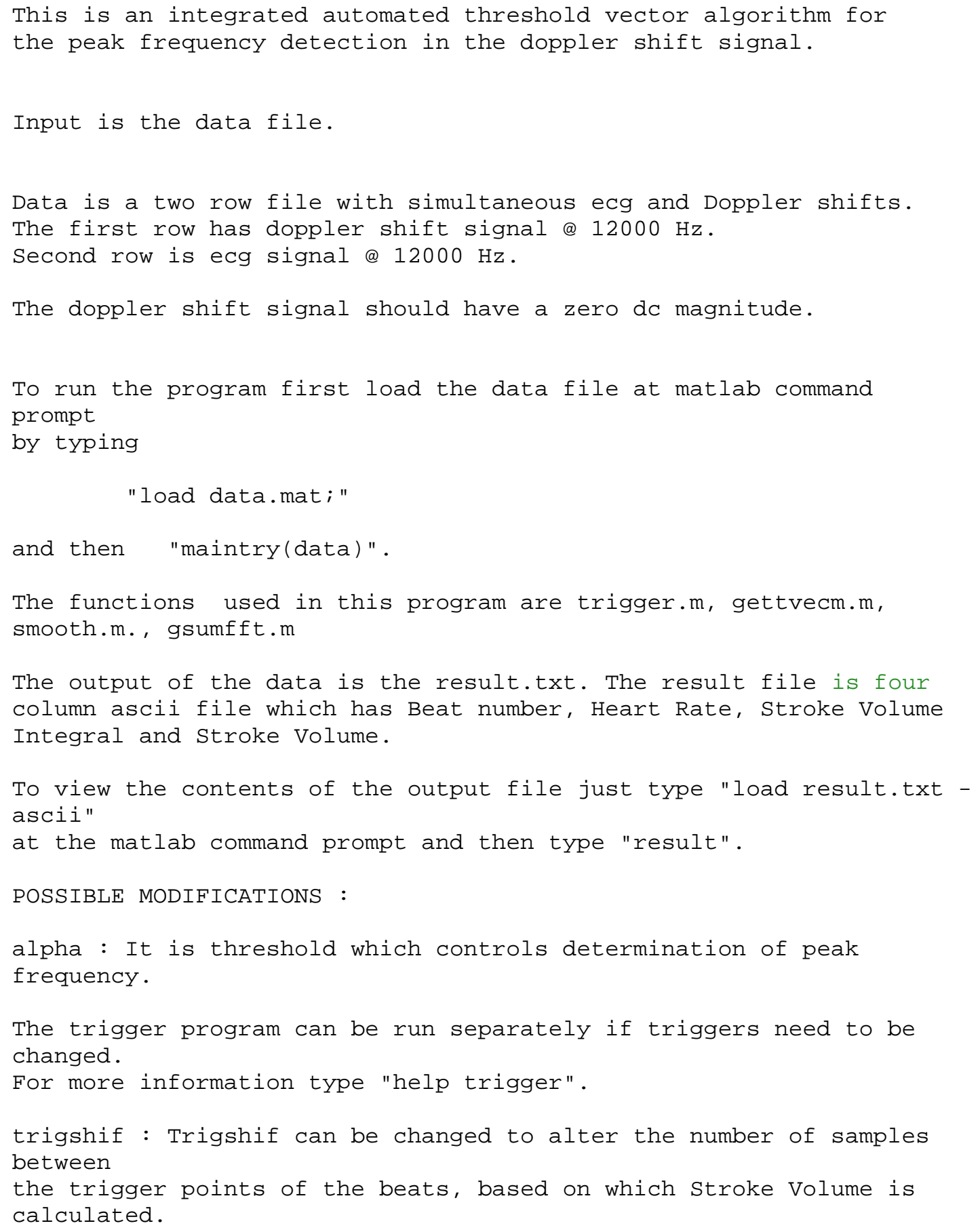




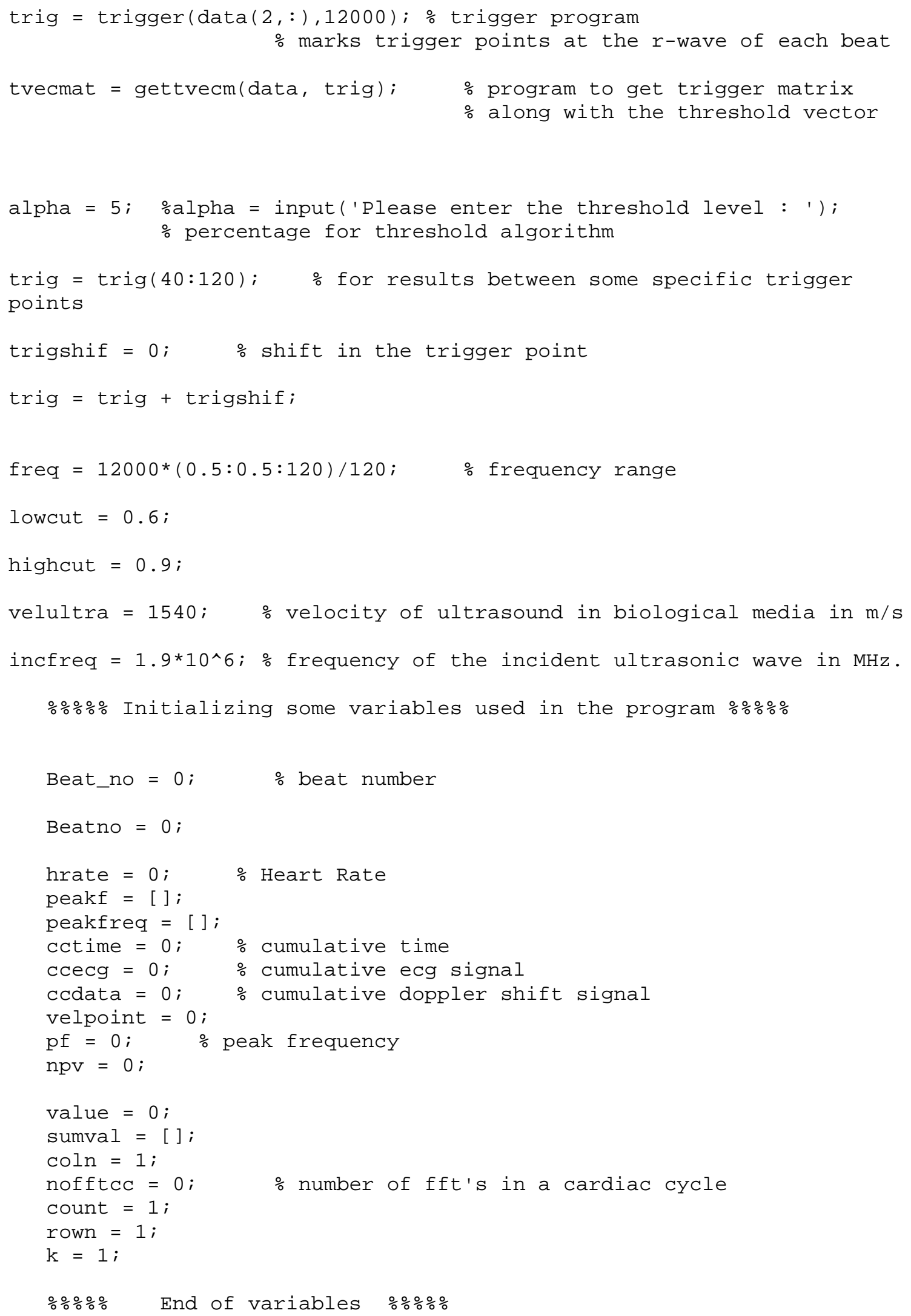




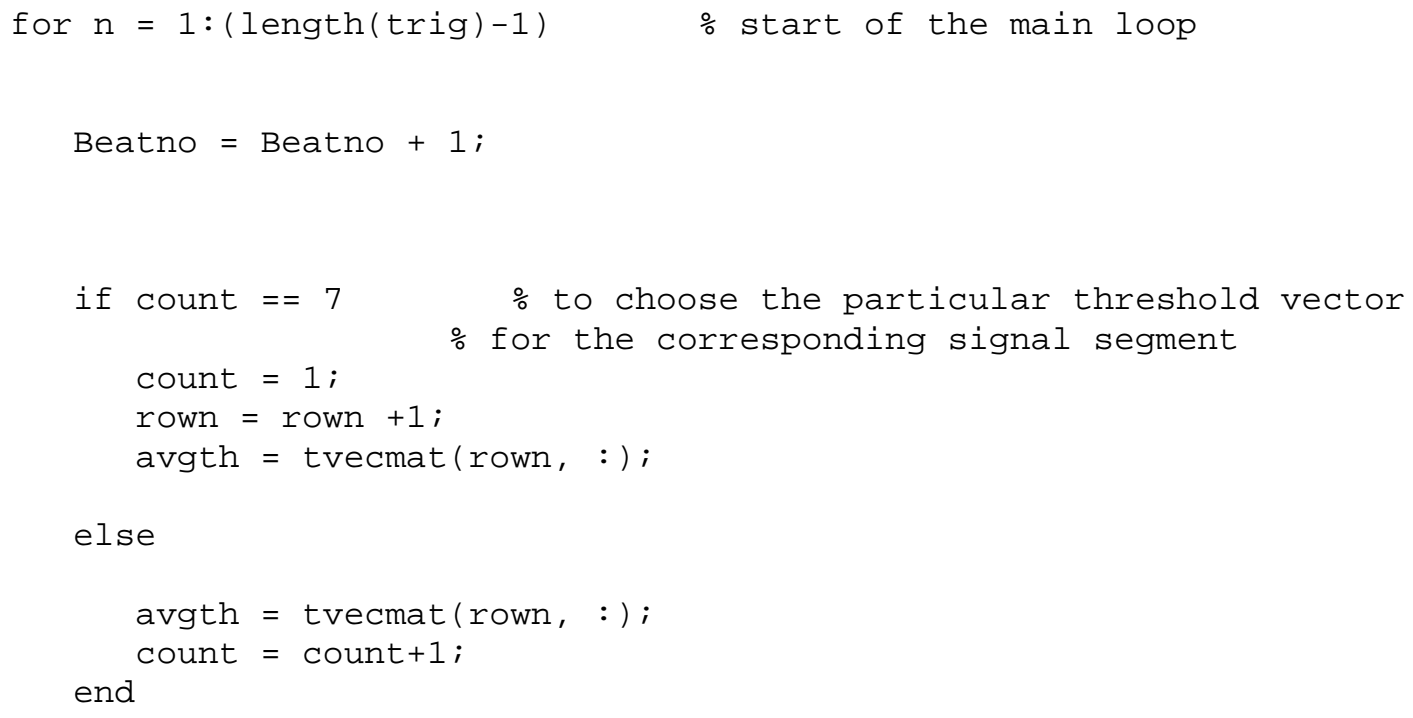




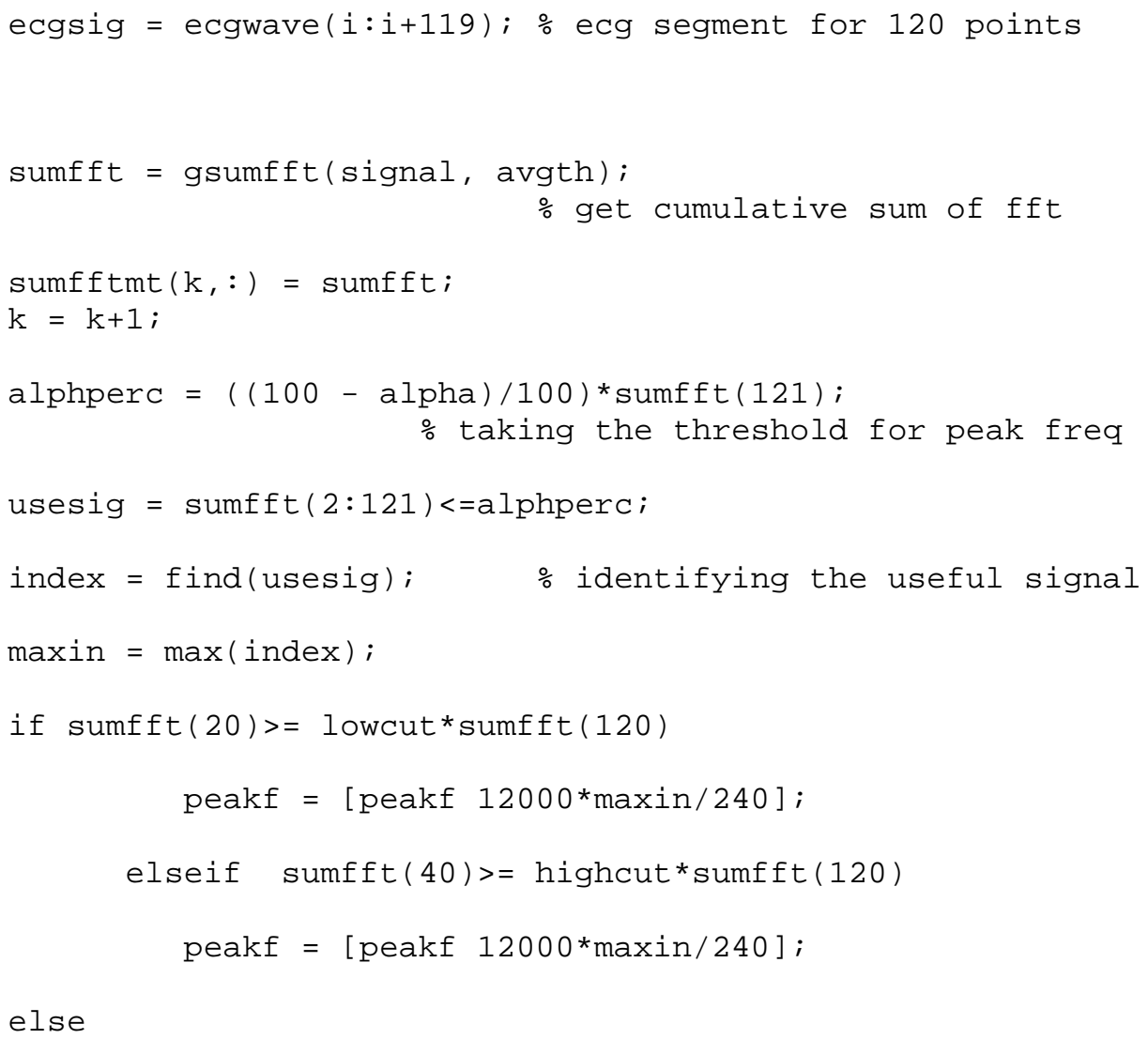

end 


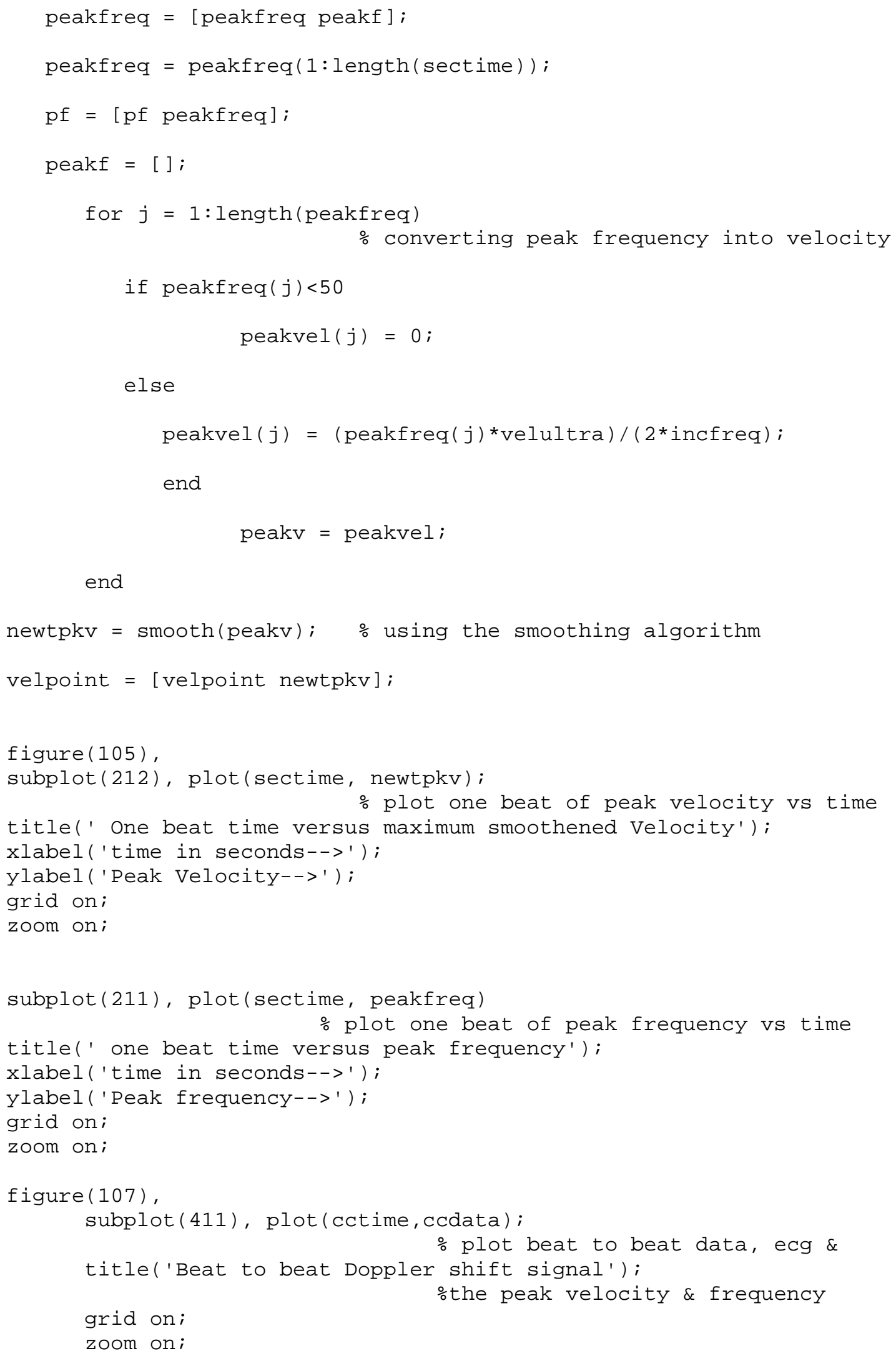




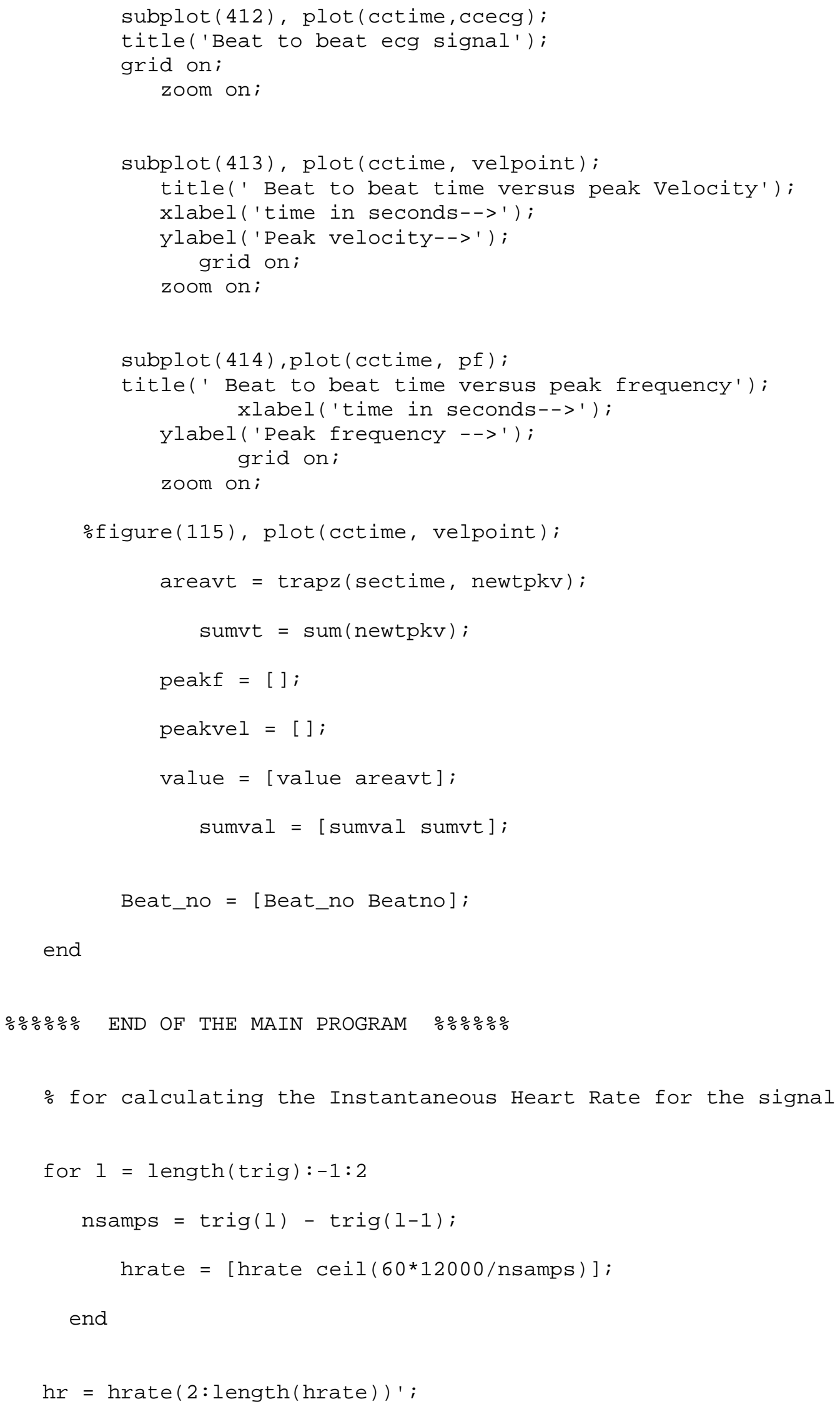




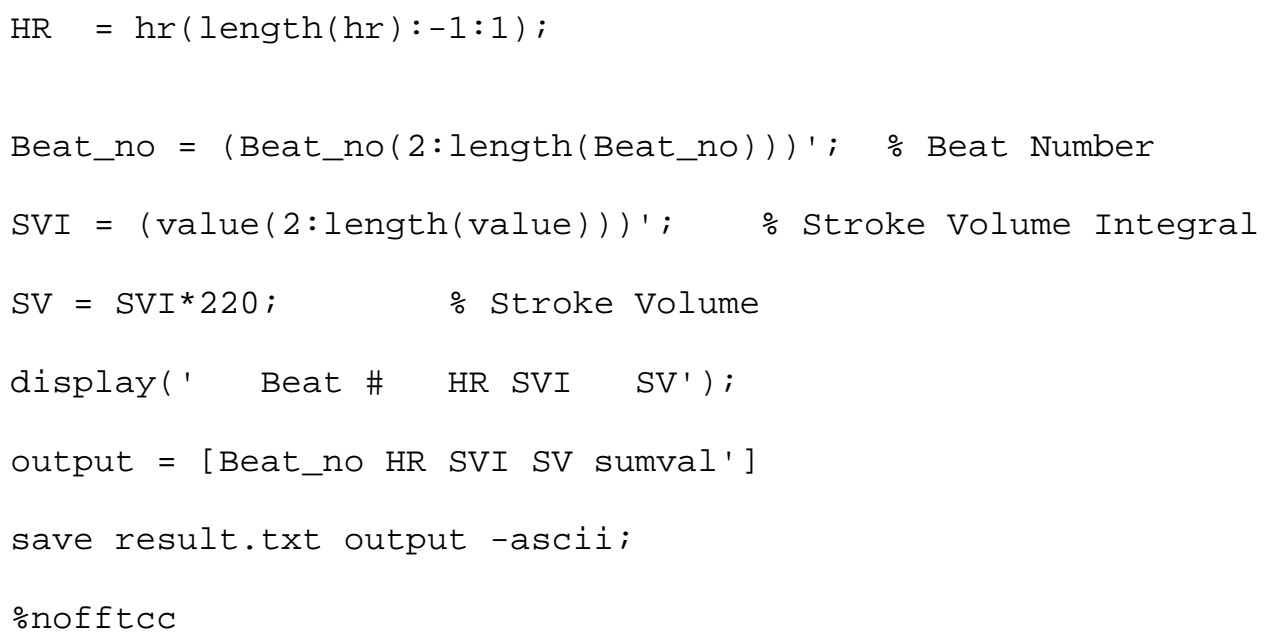




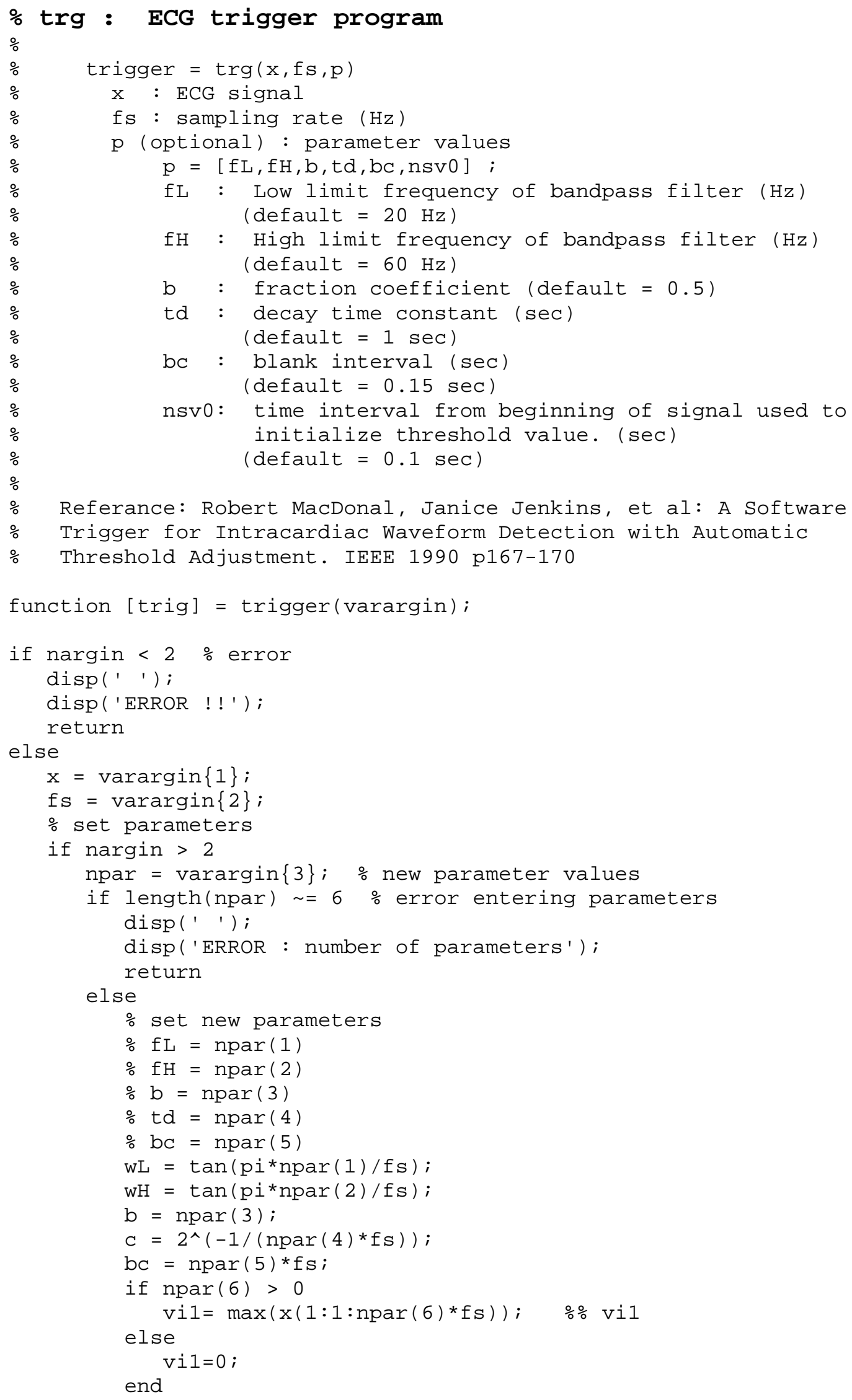




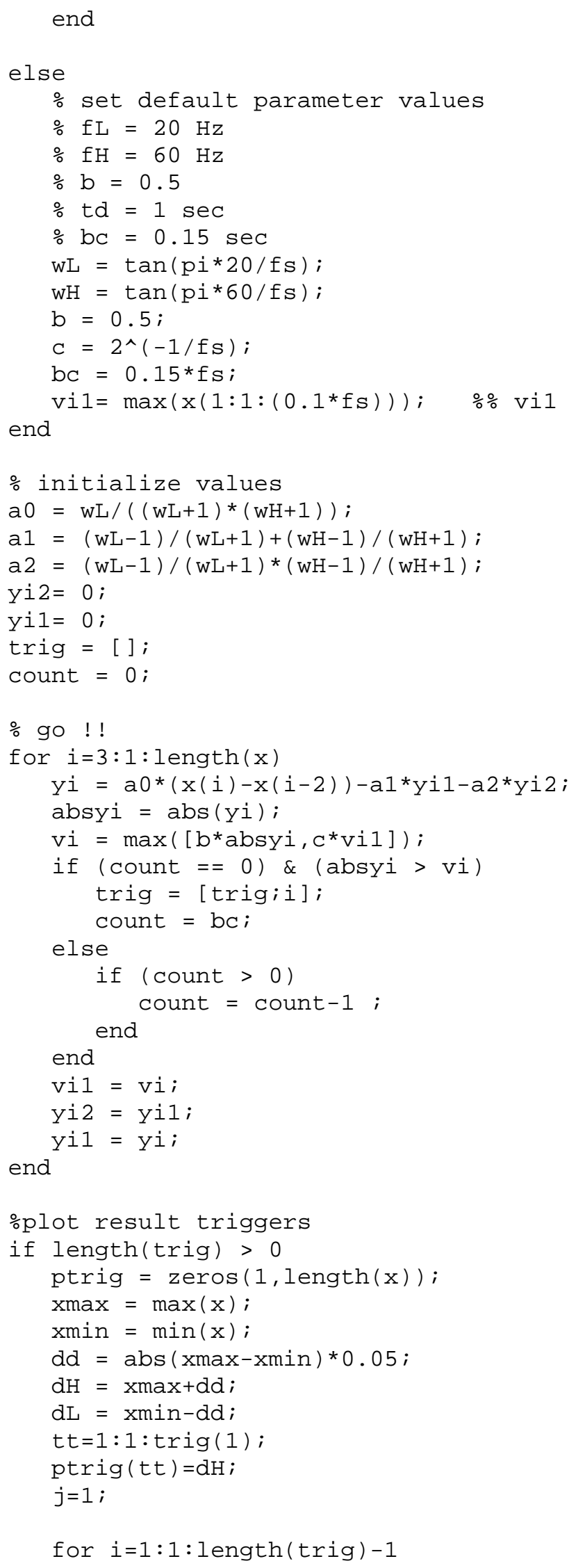




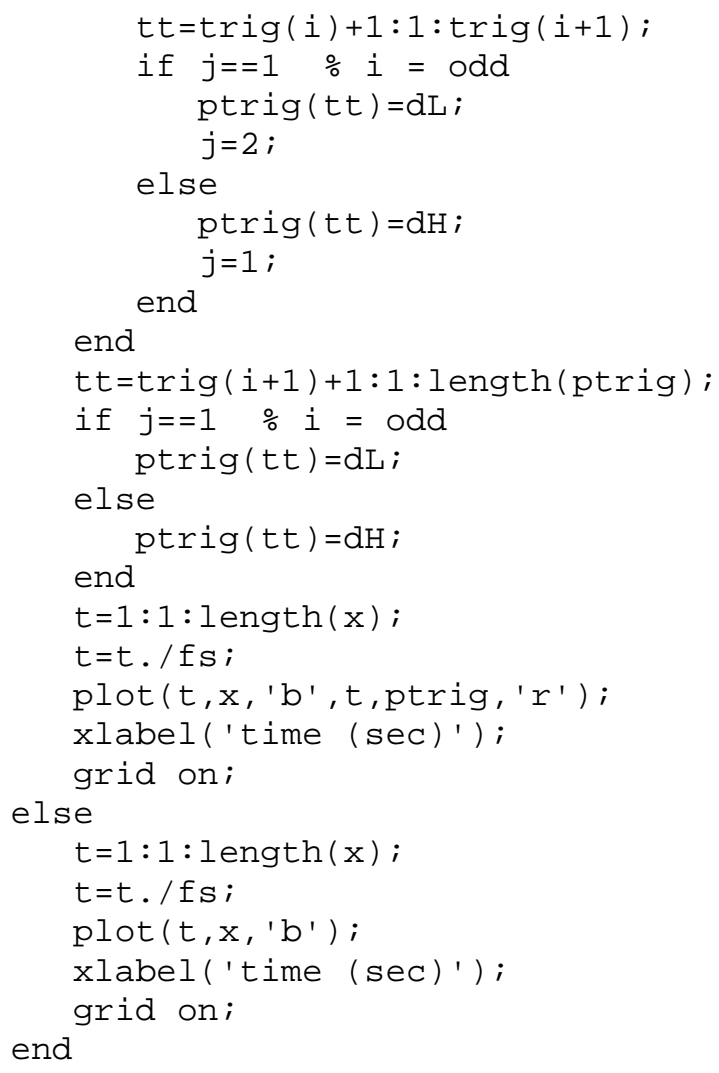




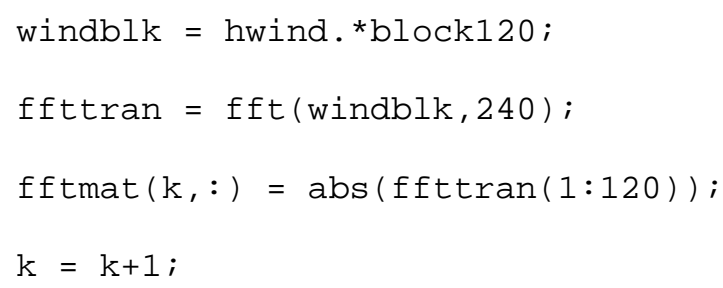




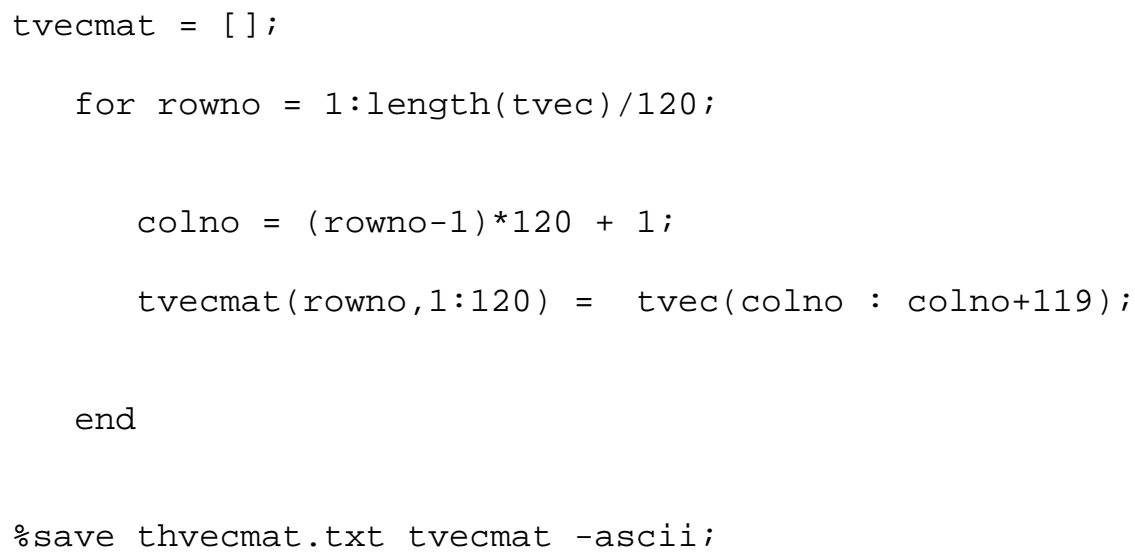




\section{function [sumfft] = gsumfft (signal, avgth)}

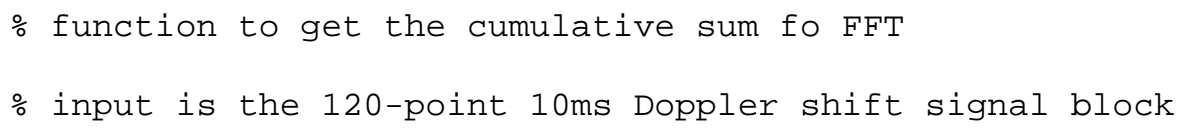




\section{function [newx] $=\operatorname{smooth}(x)$}

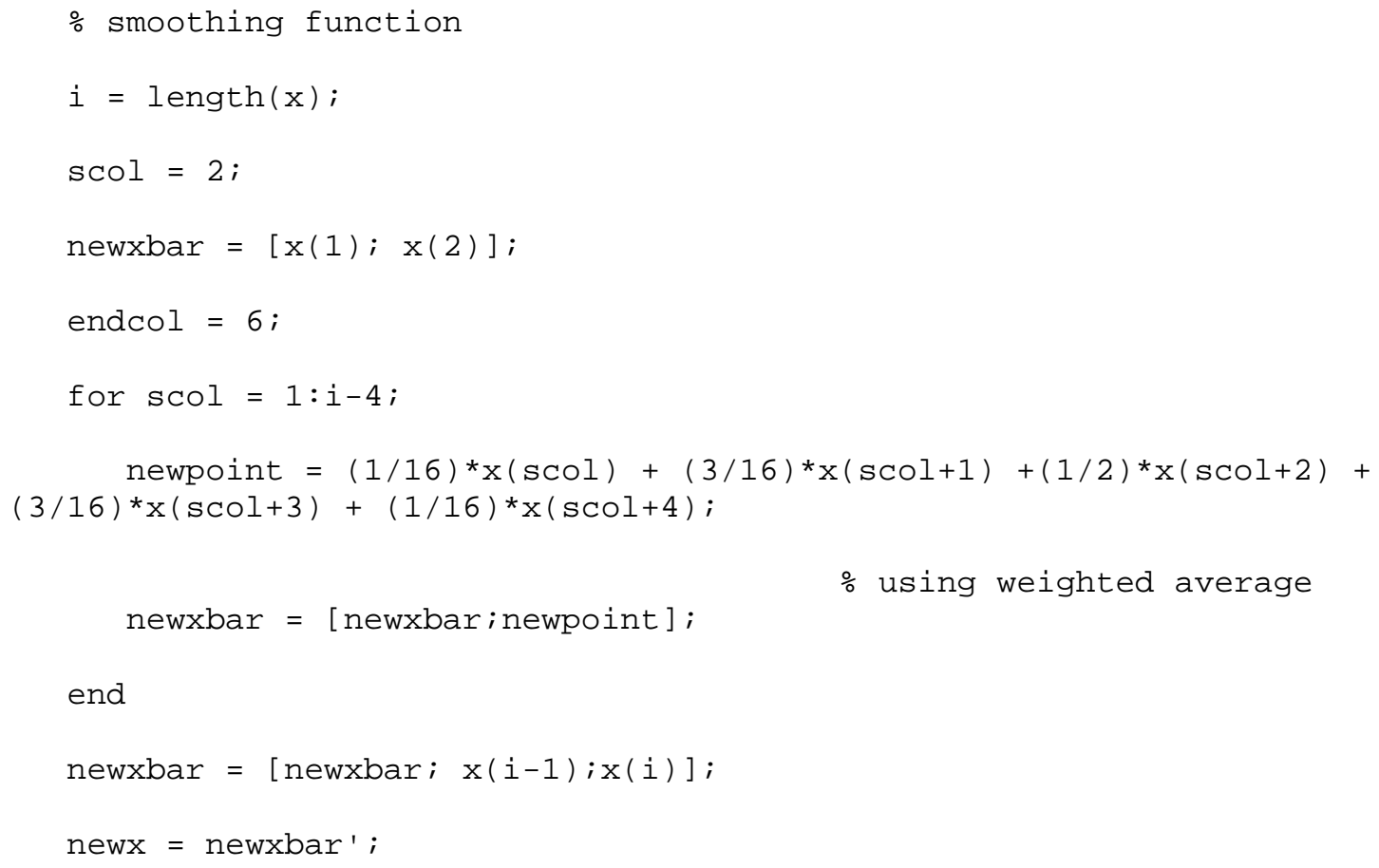




\section{APPENDIX (2): Flowcharts}




\section{Flow chart for Hybrid Percentile algorithm}

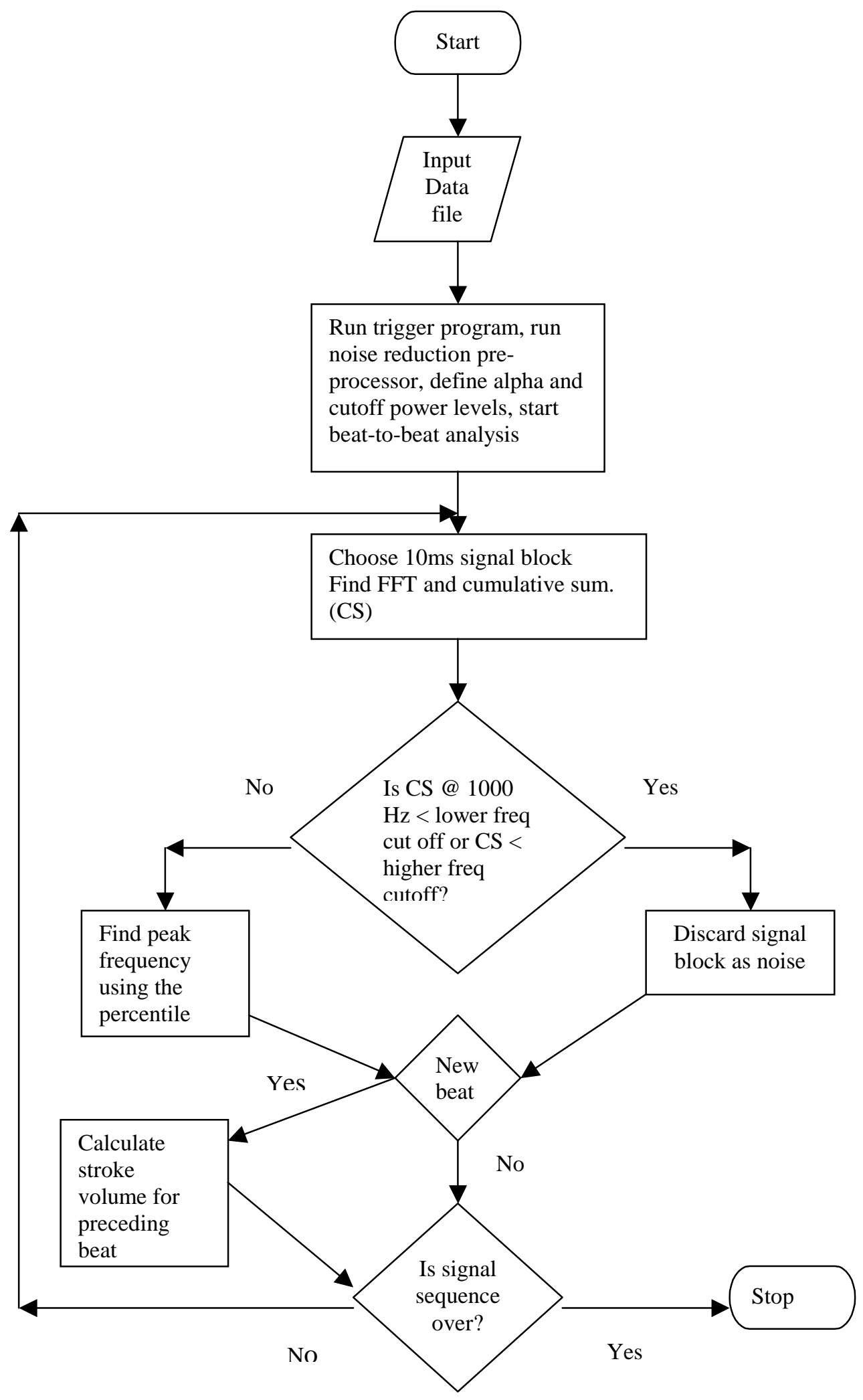




\section{Flow chart for SNR Algorithm}

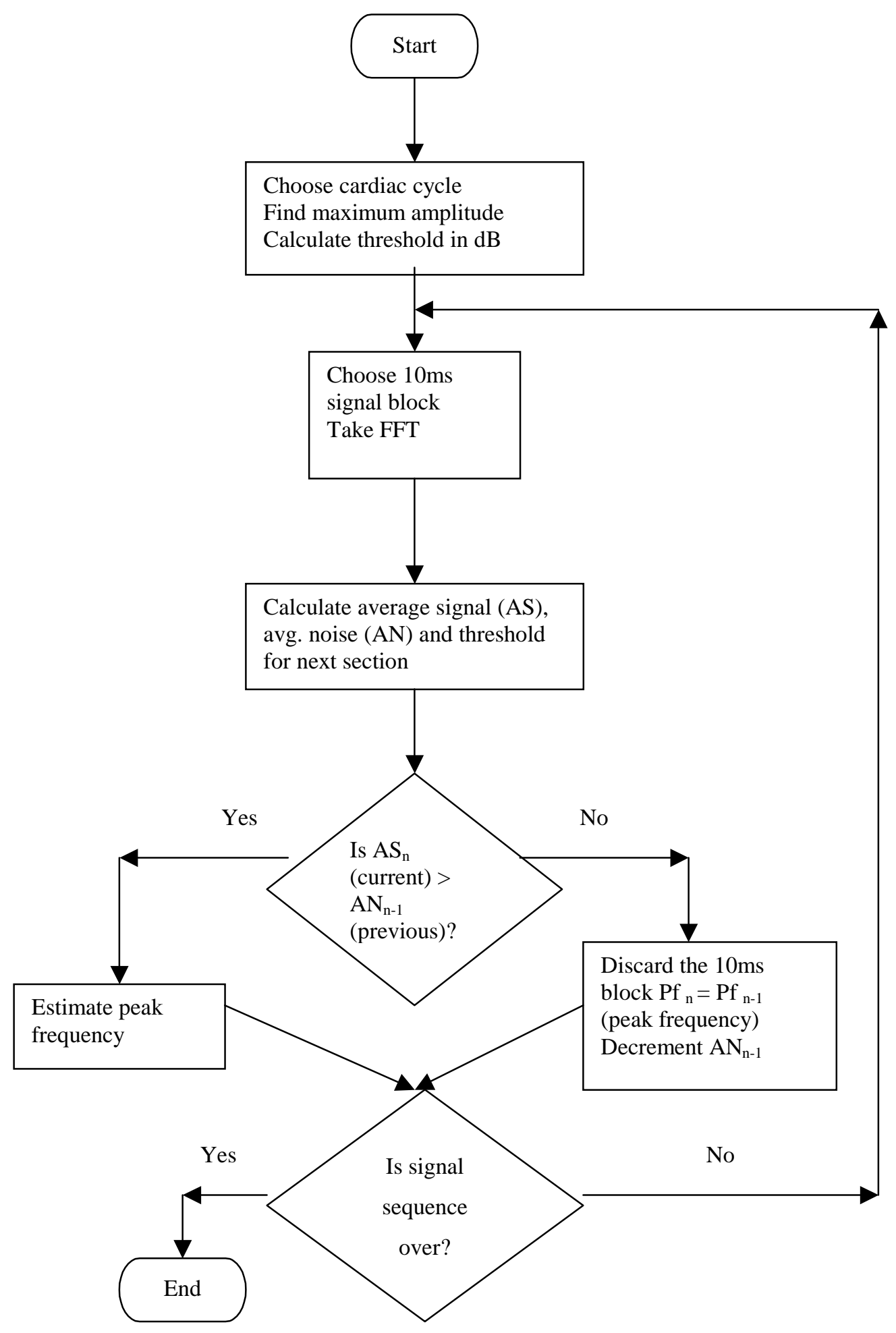


APPENDIX (3): Data 


\section{Given data}

The following information was used in the program:

Velocity of ultrasound in the biological media: $1540 \mathrm{~m} / \mathrm{s}$

Frequency of the incident ultrasound wave: $1.9 \mathrm{MHz}$.

Cross sectional area of the aorta: $2.2 \mathrm{~cm}^{2}$.

\section{Data Comparison}

\begin{tabular}{|c|c|c|c|c|c|c|c|c|c|}
\hline \multirow[b]{2}{*}{ Beat Number } & \multicolumn{3}{|c|}{ Heart Rate } & \multicolumn{3}{|c|}{$\begin{array}{l}\text { Stroke Volume } \\
\text { in } \mathrm{ml}\end{array}$} & \multirow[b]{2}{*}{ figure5.4 } & \multirow[b]{2}{*}{ figure 5.5} & \multirow[b]{2}{*}{ figure5.6 } \\
\hline & Reference & Algorithm & Reference & figure5.1 & figure 5.2 & figure5.3 & & & \\
\hline 1 & 65 & 70.00 & 51.5 & 65.31 & 50.55 & 59.25 & 35.98 & 42.93 & 48.55 \\
\hline 2 & 65 & 70.00 & 53.5 & 65.31 & 50.55 & 59.25 & 35.98 & 42.93 & 48.55 \\
\hline 3 & 71 & 71.00 & 48.6 & 65.31 & 50.55 & 59.25 & 35.98 & 42.93 & 48.55 \\
\hline 4 & 71 & 73.00 & 46.7 & 65.31 & 50.55 & 59.25 & 35.98 & 42.93 & 48.55 \\
\hline 5 & 73 & 75.00 & 50.4 & 65.31 & 50.55 & 59.25 & 35.98 & 42.93 & 48.55 \\
\hline 6 & 73 & 77.00 & 50.7 & 65.31 & 50.55 & 59.25 & 35.98 & 42.93 & 48.55 \\
\hline 7 & 76 & 78.00 & 52 & 65.31 & 50.55 & 59.25 & 35.98 & 42.93 & 48.55 \\
\hline 8 & 76 & 78.00 & 49.4 & 65.31 & 50.55 & 59.25 & 35.98 & 42.93 & 48.55 \\
\hline 9 & 76 & 77.00 & 51 & 65.31 & 50.55 & 59.25 & 35.98 & 42.93 & 48.55 \\
\hline 10 & 77 & 79.00 & 49.4 & 65.31 & 50.55 & 59.25 & 35.98 & 42.93 & 48.55 \\
\hline 11 & & 76.00 & & 72.05 & 56.15 & 65.69 & 37.50 & 44.86 & 50.60 \\
\hline 12 & 65 & 70.00 & 52.2 & 84.16 & 63.50 & 75.64 & 45.67 & 55.72 & 63.65 \\
\hline 13 & & 70.00 & & 84.16 & 63.50 & 75.64 & 45.67 & 55.72 & 63.65 \\
\hline 14 & 65 & 69.00 & 47.3 & 85.63 & 65.01 & 76.94 & 47.18 & 57.02 & 65.12 \\
\hline 15 & & 70.00 & & 84.16 & 63.50 & 75.64 & 45.67 & 55.72 & 63.65 \\
\hline 16 & 65 & 71.00 & 43 & 78.24 & 58.52 & 69.52 & 40.68 & 49.59 & 57.74 \\
\hline 17 & 65 & 73.00 & 54.8 & 72.48 & 56.36 & 66.07 & 38.52 & 46.15 & 51.97 \\
\hline 18 & 75 & 75.00 & 48 & 72.69 & 56.39 & 66.24 & 39.37 & 47.22 & 53.12 \\
\hline 19 & 75 & 76.00 & 44 & 72.05 & 56.15 & 65.69 & 37.50 & 44.86 & 50.60 \\
\hline 20 & 75 & 75.00 & 40.9 & 72.69 & 56.39 & 66.24 & 39.37 & 47.22 & 53.12 \\
\hline 21 & 75 & 76.00 & 36.3 & 67.41 & 52.38 & 61.28 & 35.98 & 42.93 & 48.55 \\
\hline 22 & 78 & 78.00 & 37.9 & 65.31 & 50.55 & 59.25 & 35.98 & 42.93 & 48.55 \\
\hline 23 & 78 & 81.00 & 38.9 & 65.31 & 50.55 & 59.25 & 35.98 & 42.93 & 48.55 \\
\hline 24 & 81 & 82.00 & 39.1 & 65.31 & 50.55 & 59.25 & 35.98 & 42.93 & 48.55 \\
\hline 25 & 85 & 85.00 & 38.5 & 65.31 & 50.55 & 59.25 & 35.98 & 42.93 & 48.55 \\
\hline 26 & 85 & 86.00 & 40.8 & 65.31 & 50.55 & 59.25 & 35.98 & 42.93 & 48.55 \\
\hline 27 & 87 & 86.00 & 40.9 & 65.31 & 50.55 & 59.25 & 35.98 & 42.93 & 48.55 \\
\hline 28 & 86 & 85.00 & 40.4 & 65.31 & 50.55 & 59.25 & 35.98 & 42.93 & 48.55 \\
\hline 29 & 79 & 83.00 & 40.5 & 65.31 & 50.55 & 59.25 & 35.98 & 42.93 & 48.55 \\
\hline 30 & 79 & 78.00 & 44.3 & 67.31 & 52.06 & 61.00 & 37.48 & 44.68 & 50.55 \\
\hline
\end{tabular}




\begin{tabular}{|c|c|c|c|c|c|c|c|c|c|}
\hline 31 & & 74.00 & & 68.87 & 53.23 & 62.37 & 38.65 & 46.05 & 52.11 \\
\hline 32 & 72 & 71.00 & 41.7 & 68.87 & 53.23 & 62.37 & 38.65 & 46.05 & 52.11 \\
\hline 33 & 72 & 70.00 & 36.2 & 68.87 & 53.23 & 62.37 & 38.65 & 46.05 & 52.11 \\
\hline 34 & 69 & 70.00 & 42.8 & 68.87 & 53.23 & 62.37 & 38.65 & 46.05 & 52.11 \\
\hline 35 & 69 & 71.00 & 47.2 & 68.87 & 53.23 & 62.37 & 38.65 & 46.05 & 52.11 \\
\hline 36 & 73 & 73.00 & 42.9 & 68.87 & 53.23 & 62.37 & 38.65 & 46.05 & 52.11 \\
\hline 37 & 73 & 77.00 & 47.8 & 69.77 & 53.90 & 63.15 & 39.32 & 46.83 & 53.00 \\
\hline 38 & 80 & 80.00 & 43.2 & 65.31 & 50.55 & 59.25 & 35.98 & 42.93 & 48.55 \\
\hline 39 & 81 & 81.00 & 50.2 & 65.31 & 50.55 & 59.25 & 35.98 & 42.93 & 48.55 \\
\hline 40 & 82 & 83.00 & 43.8 & 65.31 & 50.55 & 59.25 & 35.98 & 42.93 & 48.55 \\
\hline 41 & 84 & 84.00 & 46 & 65.31 & 50.55 & 59.25 & 35.98 & 42.93 & 48.55 \\
\hline 42 & & 86.00 & & 65.31 & 50.55 & 59.25 & 35.98 & 42.93 & 48.55 \\
\hline 43 & 85 & 87.00 & 41.3 & 65.31 & 50.55 & 59.25 & 35.98 & 42.93 & 48.55 \\
\hline 44 & 85 & 89.00 & 36.7 & 65.31 & 50.55 & 59.25 & 35.98 & 42.93 & 48.55 \\
\hline 45 & 87 & 89.00 & 45.7 & 65.31 & 50.55 & 59.25 & 35.98 & 42.93 & 48.55 \\
\hline 46 & 87 & 88.00 & 44.2 & 65.31 & 50.55 & 59.25 & 35.98 & 42.93 & 48.55 \\
\hline 47 & & 85.00 & & 65.31 & 50.55 & 59.25 & 35.98 & 42.93 & 48.55 \\
\hline 48 & 80 & 81.00 & 42.9 & 65.31 & 50.55 & 59.25 & 35.98 & 42.93 & 48.55 \\
\hline 49 & 80 & 77.00 & 45.9 & 71.31 & 55.70 & 65.03 & 37.78 & 44.76 & 50.48 \\
\hline 50 & 76 & 75.00 & 44.6 & 70.93 & 55.47 & 64.65 & 38.38 & 45.37 & 51.12 \\
\hline 51 & 76 & 74.00 & 46.9 & 71.78 & 56.24 & 65.46 & 38.98 & 45.98 & 51.76 \\
\hline 52 & 74 & 74.00 & 45.2 & 71.78 & 56.24 & 65.46 & 38.98 & 45.98 & 51.76 \\
\hline 53 & 75 & 75.00 & 46.7 & 70.93 & 55.47 & 64.65 & 38.38 & 45.37 & 51.12 \\
\hline 54 & 75 & 78.00 & 45 & 67.14 & 52.06 & 61.03 & 35.98 & 42.93 & 48.55 \\
\hline 55 & 80 & 80.00 & 46.3 & 65.31 & 50.55 & 59.25 & 35.98 & 42.93 & 48.55 \\
\hline 56 & & 81.00 & & 65.31 & 50.55 & 59.25 & 35.98 & 42.93 & 48.55 \\
\hline 57 & 81 & 81.00 & 43 & 65.31 & 50.55 & 59.25 & 35.98 & 42.93 & 48.55 \\
\hline 58 & 81 & 84.00 & 40.9 & 65.31 & 50.55 & 59.25 & 35.98 & 42.93 & 48.55 \\
\hline 59 & 85 & 85.00 & 41.6 & 65.31 & 50.55 & 59.25 & 35.98 & 42.93 & 48.55 \\
\hline 60 & 86 & 86.00 & 38.2 & 65.31 & 50.55 & 59.25 & 35.98 & 42.93 & 48.55 \\
\hline 61 & & 88.00 & & 65.31 & 50.55 & 59.25 & 35.98 & 42.93 & 48.55 \\
\hline 62 & 84 & 87.00 & 44.1 & 65.31 & 50.55 & 59.25 & 35.98 & 42.93 & 48.55 \\
\hline 63 & 84 & 85.00 & 42.9 & 65.31 & 50.55 & 59.25 & 35.98 & 42.93 & 48.55 \\
\hline 64 & 81 & 83.00 & 39.2 & 65.31 & 50.55 & 59.25 & 35.98 & 42.93 & 48.55 \\
\hline 65 & 79 & 81.00 & 40 & 65.31 & 50.55 & 59.25 & 35.98 & 42.93 & 48.55 \\
\hline 66 & & 81.00 & & 65.31 & 50.55 & 59.25 & 35.98 & 42.93 & 48.55 \\
\hline 67 & 81 & 81.00 & 41.5 & 65.31 & 50.55 & 59.25 & 35.98 & 42.93 & 48.55 \\
\hline 68 & 81 & 81.00 & 44.6 & 65.31 & 50.55 & 59.25 & 35.98 & 42.93 & 48.55 \\
\hline 69 & 80 & 81.00 & 48.5 & 65.31 & 50.55 & 59.25 & 35.98 & 42.93 & 48.55 \\
\hline 70 & 80 & 83.00 & 41.2 & 65.31 & 50.55 & 59.25 & 35.98 & 42.93 & 48.55 \\
\hline 71 & 83 & 85.00 & 40.4 & 65.31 & 50.55 & 59.25 & 35.98 & 42.93 & 48.55 \\
\hline 72 & 85 & 86.00 & 46.3 & 65.31 & 50.55 & 59.25 & 35.98 & 42.93 & 48.55 \\
\hline 73 & & 88.00 & & 65.31 & 50.55 & 59.25 & 35.98 & 42.93 & 48.55 \\
\hline 74 & 89 & 89.00 & 42.8 & 65.31 & 50.55 & 59.25 & 35.98 & 42.93 & 48.55 \\
\hline 75 & 89 & 90.00 & 44.8 & 65.31 & 50.55 & 59.25 & 35.98 & 42.93 & 48.55 \\
\hline 76 & 91 & 91.00 & 39.9 & 65.31 & 50.55 & 59.25 & 35.98 & 42.93 & 48.55 \\
\hline 77 & 92 & 92.00 & 42.1 & 65.31 & 50.55 & 59.25 & 35.98 & 42.93 & 48.55 \\
\hline 78 & 92 & 93.00 & 39.3 & 65.31 & 50.55 & 59.25 & 35.98 & 42.93 & 48.55 \\
\hline 79 & 89 & 91.00 & 45 & 65.31 & 50.55 & 59.25 & 35.98 & 42.93 & 48.55 \\
\hline 80 & 87 & 89.00 & 42.5 & 65.31 & 50.55 & 59.25 & 35.98 & 42.93 & 48.55 \\
\hline
\end{tabular}

